\title{
Integrated Sensing \& Controls for Coal Gasification - Development of Model-Based Controls for GE's Gasifier \& Syngas Cooler
}

\section{Final Technical Report}

Reporting Period: July 2007 - December 2010

\author{
Principal Author: Aditya Kumar
}

Issued: March 2011

DOE Award Number: DE-FC26-07NT43094

Submitted by:

GE Global Research

One Research Circle Niskayuna NY 12309 
"This report was prepared as an account of work sponsored by an agency of the United States Government. Neither the United States Government nor any agency thereof, nor any of their employees, makes any warranty, express or implied, or assumes any legal liability or responsibility for the accuracy, completeness, or usefulness of any information, apparatus, product, or process disclosed, or represents that its use would not infringe privately owned rights. Reference herein to any specific commercial product, process, or service by trade name, trademark, manufacturer, or otherwise does not necessarily constitute or imply its endorsement, recommendation, or favoring by the United States Government or any agency thereof. The views and opinions of authors expressed herein do not necessarily state or reflect those of the United States Government or any agency thereof." 


\section{Table of Contents}

\section{ABSTRACT}

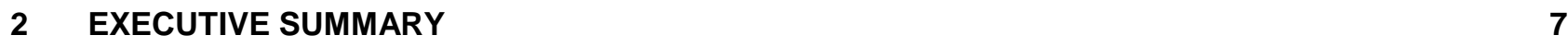

2.1 TASK 1 - MODELING FOR GASIFICATION SECTION 2

2.2 TASK 1 - SENSOR IMPLEMENTATION IN RSC AT TECO PLANT

2.3 TASK 2 - SENSING SYSTEM DESIGN

2.4 TASK 3 - CONTROL SYSTEM DESIGN 29

$\begin{array}{llr}3 & \text { INTRODUCTION } & 10\end{array}$

4 TASK 1 - MODELING \& SENSOR IMPLEMENTATION 13

4.1 MODELING

4.1.1 Gasifier 16

4.1.1.1 Slag Flow Model 20

4.1.2 Radiant Syngas Cooler (RSC) and High Pressure (HP) Steam Drum 21

4.1.3 RSC Quench/Sump and Scrubber 24

4.1.4 RSC Pre-heating $\quad 24$

4.1.5 Gasifier Pre-heating $\quad 25$

4.2 SENSOR IMPLEMENTATION IN TECO IGCC PLANT

4.2.1 Lab Testing for Temperature and Strain Sensing using Fiber Optic Sensors 28

4.2.1.1 Lab Tests for Fiber Optic Temperature Sensing 28

4.2.1.2 Lab Tests for Fiber Optic Strain Sensing 31

4.2.2 Temperature \& Strain Sensor Packaging and Installation 36

4.2.3 RSC Data Collection 38

4.3 Model Validation With TECO IGCC Plant Data for Nominal Operation 43

$5 \quad$ TASK 2 - SENSING SYSTEM DESIGN 46

5.1 MODEL-BASED ANALYSIS FOR SENSING AND ESTIMATION

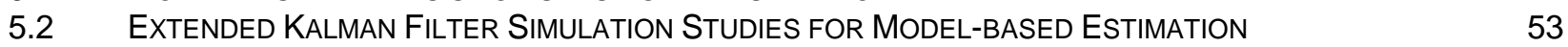

6 TASK 3 - CONTROL SYSTEM DESIGN $\quad 64$

6.1 MPC FOR STARTUP PRE-HEATING OPTIMIZATION 66

6.2 MPC FOR NOMINAL OPERATION OPTIMIZATION WITH IDEAL SENSORS

6.2.1 MPC for Nominal Operation Optimization with Coal

6.2.2 MPC for Nominal Operation Optimization with Coal-Petcoke Blend 76

6.3 INTEGRATED MPC AND EKF FOR NOMINAL OPERATION OPTIMIZATION

6.3.1 Nominal Operation Optimization with Coal 80

6.3.2 Nominal Operation Optimization with Coal-Petcoke Blend 86

7 CONCLUSIONS $\quad 91$

$\begin{array}{lll}7.1 & \text { DYNAMIC MODELING FOR SENSING AND CONTROLS DESIGN } & 91\end{array}$

7.2 ADVANCED MODEL-BASED SENSING

\begin{tabular}{ll}
7.3 & ADVANCED MODEL-BASED CONTROL \\
\hline
\end{tabular}

8 ABBREVIATION 


\section{List of Figures}

Figure 1: Program overview - team, scope and objectives.

Figure 2: Integrated advanced model-based sensing and controls system for gasification section. 12

Figure 3: Gasification section. $\quad 14$

Figure 4: Operation modes for gasification section and dynamic model. 15

Figure 5: Model reduction approach for 1-D gasifier model. 17

Figure 6: Dominant spatial modes (first 15) for the $\mathrm{C}=6$ species modeled as combinations of these spatial modes in the reduced-order model. $\quad 18$

Figure 7: Comparison of full-order (blue $\mathrm{x}$ ) and reduced-order (red circles) simulation results for gasifier exit temperature and unconverted carbon flow rate, and corresponding errors between full- and reducedorder models.

Figure 8: Comparison of full-order (blue $\mathrm{x}$ ) and reduced-order (red circles) simulation results for some syngas component compositions at gasifier exit, and corresponding errors between full- and reduced-order models. $\quad 19$

Figure 9: Gasifier slag flow model structure. 20

Figure 10: 1-D (axial) RSC heat transfer model for counter-current flow of hot syngas and water/steam and heat exchange.

Figure 11: Steady-state profiles for steam mass fraction, water and syngas temperature in RSC for base-load and half-load conditions in clean RSC and base-load conditions with fully fouled RSC. 22

Figure 12: RSC HP steam drum model structure with steam holdup under the water level. 23

Figure 13: Simulation run with 5\% drop in HP steam drum pressure showing transient drum level surge due to flow rate variation in RSC tubes and variation in steam volume fraction under water level during the transient.

Figure 14: RSC pre-heating with steam.

Figure 15: Pre-heating temperature profiles in RSC - comparison of model outputs and TECO IGCC plant startup operation data.

Figure 16: Transient temperature profiles in the gasifier refractory layers and metal shell during pre-heating obtained by transient thermal model.

Figure 17: Transient profiles for tensile and compressive stresses as fraction of respective strengths in the innermost refractory layer obtained by the ANSYS ${ }^{\circ}$ model.

Figure 18: Lab setup for fiber optic temperature sensor short-term \& long-term testing. 28

Figure 19: Signal characteristics from two prototype FBG temperature sensors 29

Figure 20: Short term temperature measurement errors for two FBG sensor prototypes.

Figure 21: Short-term performance for repeatability of temperature measurement for two FBG sensor prototypes.

Figure 22: Longer term testing for survivability and stability of temperature measurement using FBG sensor prototype I

Figure 23: Lab setup for high-temperature fiber-optic strain sensor.

Figure 24: FBG strain sensor options (a) metalized fibers inside a bi-metallic tube packaging, and (b) metalized fiber directly mounted to substrate (rod).

Figure 25: Performance of metalized fiber FBG strain sensor directly mounted on the rod in the hot section at
temperatures up to $700 \mathrm{~F}$, compared to a resistive strain sensor mounted on the cold end of the rod.

Figure 26: Performance of metalized fiber FBG strain sensor directly mounted on the rod in the hot section at temperatures between $800 \mathrm{~F} \& 1000 \mathrm{~F}$, compared to a resistive strain sensor mounted on the cold end of the rod.

Figure 27: Lab test results with Ni-plated fiber (including FBG area) in SS tubing. Mechanical strain measurement at (a) $800 \mathrm{~F}$ and (b) $600 \mathrm{~F}$ using calibration obtained at $800 \mathrm{~F}$.

Figure 28: Radial temperature sensor hexoloy package with integrated Type B thermocouples and FBG sensors used at levels 7 and 10 in RSC.

Figure 29: Four hexoloy radial temperature probes with integrated Type B TCs and FBG sensors (two probes on right) and single Type B TCs (two probes on left).

Figure 30: Soot-blower lances with temperature sensor package installed in TECO IGCC RSC.

Figure 31: Spectrum data from fiber optic sensors with 10 FBG sensors for each soot blower lance installed in the TECO IGCC RSC. 
Figure 32: Peak intensity and normalized temperature from 10 FBG sensors at SBL level 7.

Figure 33: Peak intensity and normalized temperatures from FBG Sensors at level 10.

Figure 34: Normalized temperature from TCs and FBG sensors at level 10.

Figure 35: Spectrum data from two fiber optic FBG sensors used for strain sensing.

Figure 36: Measured (normalized) temperature in the RSC dome.

Figure 37: Calculated mechanical strain from FBG sensor, during stable thermal operation.

Figure 38: Radial distribution for temperature (left) and syngas axial velocity (right) assumed at Level $10 . \quad 46$

Figure 39: Advanced sensing system for gasification section.

Figure 40: Estimation errors for important un-measured variables due to modeling error and with/without sensor measurement errors.

Figure 41: Estimation errors for important un-measured variables due to sensor measurement error and with/without modeling errors.

Figure 42: The sensitivity of the estimation error of gasifier exit syngas temperature to the biases of the baseline sensor set. The important biases are drum steam flow, oxygen flow, $\mathrm{CO}_{2}$ flow, throughput, $\mathrm{O}_{2}$ flow and purity, and fuel composition.

3: The sensitivity of the estimation error of gasifier exit syngas temperature to the model parameters The important parameters are drum feed water enthalpy, ash-to-slag transfer rate, heterogeneous reaction time constant, quench and scrubber vapor-liquid equilibrium temperature mismatches, and drum circulation pump's head/flow characteristics.

Figure 44: Open-loop implementation of the extended Kalman filter, where EKF is running in parallel to the MPC with state feedback from the plant.

Figure 45: Estimation of key unmeasured process variables during load changes.

Figure 46: Estimation of unknown model parameters during load changes.

Figure 47: Estimation of key unmeasured process variables during oxygen feed changes.

Figure 48: Estimation of unknown model parameters during changes in oxygen feed.

Figure 49: Performance of the preemptive constrained EKF compared with the open-loop estimation for the four critical unmeasured variables. EKF estimate shows close to zero-mean while open-loop estimates have significant non-zero mean.

Figure 50: Determination of the outliers in the estimation of the gasifier temperature in the test runs. Each point represents the steady state estimation error of the gasifier temperature in that specific run. Simulation runs $19,27,31,37,43$, and 53 were the outliers.

Figure 51: Performance of the PLS regression explaining the relationship between the biases and the gasifier temperature estimation error. The PLS regression model explains more than $95 \%$ of the variance observed in the estimation error data.

Figure 52: The cumulative loadings of the biases on the first three modes of the PLS regression.

Figure 53: The comparison of the estimation of gasifier temperature in the test cases before and after the elimination of the $\mathrm{O}_{2}$ flow rate bias.

Figure 54: Schematic representation of the Integrated Sensing \& Control Solution using EKF \& MPC. 65

Figure 55: MPC implementation.

Figure 56: Gasifier startup preheating and thermal stresses - Open-loop schedule and MPC optimized profile. Design case with contact between refractory bricks.

Figure 57: Gasifier startup preheating and thermal stresses - Open-loop schedule and MPC optimized profile. Design case with no contact between refractory bricks.

Figure 58: MPC optimized pre-heating of RSC.

Figure 59: Summary of steady state and transient optimization with MPC using different control inputs

Figure 60: MPC performance for oxygen use minimization at $50 \%$ load operation with coal.

Figure 61: MPC performance for faster ramp up during load transients between $50 \%$ and $100 \%$ load for operation with coal.

Figure 62: MPC optimization for nominal operation with coal during steady-state operation at part-load and baseload, and during turndown.

Figure 63: Comparison of open-loop operation (green) with change in petcoke fraction with MPC operation (blue).

Figure 64: MPC optimized operation at part-load with 50\% coal-petcoke blend showing up to $5.8 \%$ variation in oxygen use between operation with maximum (green) and minimum (blue) oxygen consumption while maintaining the power output at $50 \%$.

Figure 65: MPC optimized operation at 50\% part-load and 100\% baseload and during turndown with 50\%
petcoke-coal blend.

Figure 65: MPC optimized operation at 50\% part-load and 100\% baseload and during turndown with 50\%
petcoke-coal blend.

Figure 66: Schematic of Integrated EKF and MPC. The EKF uses sensor measurements to update the states and parameters for reduced order model used in MPC. 
Figure 67: Integrated sensing and control system closed loop response to load change from 100\% -50\%$100 \%$ with sensor and parameter errors with coal. The MW output and scrubber pressure (blue) track the reference profile (red) with $25 \%$ (ramp down) to $20 \%$ (ramp up) faster ramp rate compared to nominal.

Figure 68: Integrated sensing and control system closed loop response to load change with coal from $100 \%$ $50 \%-100 \%$ with one combination of random sensor biases and parameter error.

Figure 69: Monte Carlo simulation results for Net Electrical output gain at the base load condition and oxygen saving at $50 \%$ part load condition for various random combination of sensor error (noise and bias) and model parameter error (gasifier kinetics and RSC fouling) using the integrated sensing and control system for load changes from 100\%-50\%-100\%.

Figure 70: Monte Carlo simulation results for gasifier kinetics and RSC fouling for various random combination of sensor bias and model parameter errors for the integrated sensing and control system closed loop system to load change from $100 \%-50 \%-100 \%$.

Figure 71: Integrated sensing and control system closed loop response to load changes from 100\%-50\%$100 \%$ with sensor and parameter errors for coal-petcoke blend. The MW output (blue) track the MW reference (red) with $25 \%$ (ramp down)-20\% (ramp up) faster ramp rate compared to nominal.

Figure 72: Integrated sensing and control system closed loop response to load changes from 100\%-50\%$100 \%$ for coal-petcoke blend with one combination of random sensor bias and parameter errors. 88

Figure 73: Monte Carlo simulation results for normalized oxygen saving at $50 \%$ part load condition with various random combination of sensors error (noise and bias) and model parameters error (gasifier kinetics and RSC fouling) for the integrated sensing and control system to load changes from $100 \%$ $50 \%-100 \%$.

Figure 74: Monte Carlo simulation results for gasifier kinetics and RSC fouling for various random combination of sensor noise and model parameters error for the Integrated sensing and control system closed loop system to load changes from 100\%-50\%-100\%. 


\section{Abstract}

This report summarizes the achievements and final results of this program. The objective of this program is to develop a comprehensive systems approach to integrated design of sensing and control systems for an Integrated Gasification Combined Cycle (IGCC) plant, using advanced model-based techniques. In particular, this program is focused on the model-based sensing and control system design for the core gasification section of an IGCC plant. The overall approach consists of (i) developing a first-principles physicsbased dynamic model of the gasification section, (ii) performing model-reduction where needed to derive low-order models suitable for controls analysis and design, (iii) developing a sensing system solution combining online sensors with model-based estimation for important process variables not measured directly, and (iv) optimizing the steady-state and transient operation of the plant for normal operation as well as for startup using model predictive controls (MPC). Initially, available process unit models were implemented in a common platform using Matlab/Simulink®, and appropriate model reduction and model updates were performed to obtain the overall gasification section dynamic model. Also, a set of sensor packages were developed through extensive lab testing and implemented in the Tampa Electric Company IGCC plant at Polk power station in 2009, to measure temperature and strain in the radiant syngas cooler (RSC). Plant operation data was also used to validate the overall gasification section model. The overall dynamic model was then used to develop a sensing solution including a set of online sensors coupled with model-based estimation using nonlinear extended Kalman filter (EKF). Its performance in terms of estimating key unmeasured variables like gasifier temperature, carbon conversion, etc., was studied through extensive simulations in the presence sensing errors (noise and bias) and modeling errors (e.g. unknown gasifier kinetics, RSC fouling). In parallel, an MPC solution was initially developed using ideal sensing to optimize the plant operation during startup pre-heating as well as steady state and transient operation under normal high-pressure conditions, e.g. part-load, base-load, load transition and fuel changes. The MPC simulation studies showed significant improvements both for startup pre-heating and for normal operation. Finally, the EKF and MPC solutions were coupled to achieve the integrated sensing and control solution and its performance was studied through extensive steady state and transient simulations in the presence of sensor and modeling errors. The results of each task in the program and overall conclusions are summarized in this final report.

\section{Executive Summary}

This section briefly summarizes the key accomplishments of the program. They are described in more detail for each key task in the subsequent sections.

\subsection{Task 1 - Modeling for gasification section}

The objective in Task 1 was to (i) develop a detailed transient model of the gasification section to be used for simulation studies and sensing \& control system design in Tasks 2 \& 3, and (ii) implement sensors in RSC in the TECO IGCC plant to obtain data for model validation. In particular, a dynamic model was developed for nominal, high-pressure 
operation of the gasification section for both steady state as well as transient operation like turndown (i.e., load/throughput changes) and fuel changes (coal or coal+petcoke blends). This model was then extended to encompass the post-ignition, pressure ramp-up portion of the startup process as well, where both the syngas pressure and the steam pressure are raised gradually to nominal operating pressures. In parallel, another set of models was developed for the pre-heating phase of the startup, wherein the thermal transients in the gasifier refractory and the RSC and the corresponding thermal stresses are modeled during the pre-heating operation. All the models were implemented in Matlab/Simulink® except for the gasifier preheating where a dynamic thermal model in Matlab/Simulink® was coupled with a stress calculation model in ANSYS®. For the gasifier model, a systematic model-reduction was also performed to enable fast real-time computation for simulation and controls design. The implemented models were used for all sensing and control simulation studies in this program.

\subsection{Task 1 - Sensor Implementation in RSC at TECO Plant}

In this subtask, the objective was to install sensors in the RSC in the TECO IGCC plant and obtain plant operation data that could be used for model validation. Initially, three sensor candidates were identified: (i) radial temperature profiles at levels $7 \& 10$, (ii) axial temperature profile between levels $7 \& 10$, and (iii) strain measurement in the RSC dome outside the hot syngas path. Initial lab tests for the axial temperature profile indicated high risks in packaging survivability and thus, it was not pursued for implementation. Extensive lab tests were performed in 2007 and 2008 to study and improve the performance of the optical Fiber Bragg Grating (FBG) sensors under expected thermal and strain conditions. Finally, packaging design and fabrication for the radial temperature sensor probes with integrated Type B thermocouples and optical fiber FBGs was completed in 2008, and installed in the RSC on levels 7 and 10 in the TECO IGCC plant in February/March 2009. The probe on level 10 worked very well and survived for more than the thirty days aimed for. The probe at level 7 also provided valuable temperature profile data for five days of operation after plant startup. However, on the fifth day of operation, the ceramic packaging broke off abruptly - it is suspected that the hot gas and/or slag impinged directly onto the probe causing the abrupt breakage. A set of fiber optic FBG strain sensors was also installed in the RSC dome outside the hot syngas path to monitor strain evolution over time due to gradual fouling buildup on the heat transfer area. A key challenge for this sensor was for the optical fibers to survive a large thermal strain $(\sim 6000 \mu \varepsilon)$ due to the temperature rise at startup, and thereafter accurately measure a very small mechanical strain $(\sim 50 \mu \varepsilon)$ due to fouling buildup over few weeks of operation. This sensor also worked very well for more than six weeks, matching very well with estimated mechanical strain due to gradual fouling buildup.

\subsection{Task 2 - Sensing System Design}

The objective of this task was to design a model-based sensing/estimation system that provides online measurement/estimate of process variables in the gasification section that are important for monitoring and control, e.g., gasifier temperature, carbon conversion, gasification efficiency, slag viscosity and syngas properties. To this end, initially, a linear model-based Kalman filter analysis was performed at baseload operating condition to study the performance of model-based estimation in the presence of sensing and 
modeling errors. Also, a sensitivity study was performed to identify key sensor biases and model parameter errors that contribute to the uncertainty in overall estimation accuracy. This analysis was then followed by a full nonlinear model-based estimation using an Extended Kalman Filter (EKF) to verify the performance of nonlinear estimator in the presence of errors in model parameters and sensor noise/bias. One problem with the standard EKF is that it does not enforce any constraints on the estimated state or parameter variables (e.g. mole fractions that should be between 0 and 1). To address this, the EKF implementation was extended to include constraints on all estimated variables and force them to be in the appropriate range. The performance of the constrained EKF was tested through extensive simulation studies in the presence of unknown errors in the model parameters, e.g. RSC fouling and gasifier kinetics, and random combinations of sensor biases. The EKF simulations showed that the unknown model parameters were correctly identified and updated to match simulated variations, thereby allowing accurate estimation of key process variables that are not measured but are important for monitoring and control, e.g. gasifier temperature, carbon conversion, slag viscosity and overall efficiency. The EKF was then later coupled with the MPC designed in Task 3 to achieve the integrated sensing \& control system.

\subsection{Task 3 - Control System Design}

In this task, initially a nonlinear model predictive controller (MPC) was designed and implemented to optimize the steady state and transient operation during nominal plant operation (e.g. turndown and fuel changes) as well as during startup, specifically preheating of gasifier and RSC during startup. The online real-time optimization in MPC allows a flexible optimization (e.g. ramp rate, oxygen consumption, carbon conversion, power output) depending on the operation mode. Initially in 2008 \& 2009, the MPC implementation assumed "ideal sensors" with knowledge of all important state and output variables, to identify the entitlement in achievable performance improvements for nominal operation and startup. In 2010, the EKF designed for estimation in Task 2, and MPC designed in Task 3 were integrated to obtain the final integrated sensing \& control system, and extensive simulation studies were performed in the presence of sensing and modeling errors for steady state and transient optimization.

MPC simulation studies were performed for achieving faster gasifier pre-heating subject to constraints on the thermal stresses in the refractory bricks. The simulations showed more than $20 \%$ reduction in total pre-heating time for the gasifier refractory. MPC simulations were also performed to simultaneously reduce startup time and maximum thermal tensile stresses to identify the design tradeoff between the two. Similarly, MPC simulation studies on the RSC pre-heating, subject to thermal gradient and stress constraints showed significantly faster pre-heating depending on the maximum steam flow available. Finally, MPC simulation studies were performed for nominal operation including baseload operation, turndown between baseload and $50 \%$ partload and fuel changes with up to $50 \%$ petcoke in coal-petcoke blend. Exploiting multivariable constrained optimization, MPC yielded up to $2 \%$ increased net power output at baseload and $7-10 \%$ reduction in oxygen consumption at partload for coal operation. Similarly, MPC simulations for load changes (turndown) between baseload and fifty percent load conditions indicated potentially $20 \%$ faster turndown capabilities through coordinated manipulation of multiple operating variables, while enforcing key operability constraints. These MPC studies were repeated for operation with coal-petcoke blend with up to $50 \%$ petcoke, showing similar 
improvements in turndown rates and slightly less oxygen reduction. For petcoke operation, achieving high carbon conversion becomes a significant constraint.

The initial MPC simulations were performed assuming "ideal sensors", i.e. perfect knowledge of all state and output variables. In 2010, the MPC and EKF implementations designed separately were coupled to achieve the overall integrated sensing \& control system. To this end, initially, the models used for the EKF were updated to match the reduced-order models used for MPC - owing to the high simulation cost for repeated future horizon prediction, the models for MPC were reduced to eliminate some fast dynamics like syngas pressure-flow dynamics. Initial simulation studies with the integrated EKF \& MPC indicated a need for retuning of the EKF and MPC to achieve stable and optimized operation. Final simulation results showed similar steady state and transient performance improvements in spite of sensing and model parameter errors, with slight degradation for some combinations of sensor biases. Also, the simulation studies indicated that the unknown model parameters (gasifier kinetics and RSC fouling) were more "observable" at baseload conditions, i.e. could be identified more accurately, as opposed to partload conditions, which makes sense given the higher sensitivity with respect to these parameters at higher throughput at baseload operation.

\section{Introduction}

Integrated Gasification Combined Cycle (IGCC) is emerging as a promising technology for clean and efficient power generation from coal. Over the last decade or so, several advancements have been made in coal gasification technology, through concerted efforts by DOE and the industry. GE is currently commercializing this technology with the first fullscale plant being built for Duke Energy at Edwardsport, Indiana. While IGCC holds significant promise for clean and efficient power generation from coal, there is significant opportunity to improve the plant operation for enhanced reliability, availability, efficiency and flexibility through advanced sensing and controls. An IGCC plant essentially includes a chemical plant with multiple integrated sections like the air separation unit (ASU), gasification, and syngas processing and cleanup, coupled with a combined cycle power generation plant. It is desirable to operate the IGCC plant with higher flexibility, including different fuels and varying throughput and power generation, while maintaining or improving efficiency and availability. Traditional controls and operation based on simple approaches and relying on operator experience are conservative, and thus, do not achieve the potential operation entitlement. In particular, the gasification section, which has a particularly harsh environment with high temperatures and pressures and presence of slag and corrosive elements, has limited online sensing available currently for monitoring and controls. Consequently, the operation of this section, using a combination of simple controls and operator judgment based on limited and/or infrequent measurements, is often conservative, especially for transient operations. This program focused on developing an advanced model-based integrated sensing and controls solution for improved operation of the gasification section. The overall program scope and objective and the team is shown in Figure 1. GE Global Research, in consultation with the GE Energy Gasification \& Controls teams, developed the models, and advanced model-based sensing and controls solutions. Also, specific sensor packages were developed at GE Global Research and implemented in the TECO Polk Power Station IGCC plant to obtain plant operation data. 


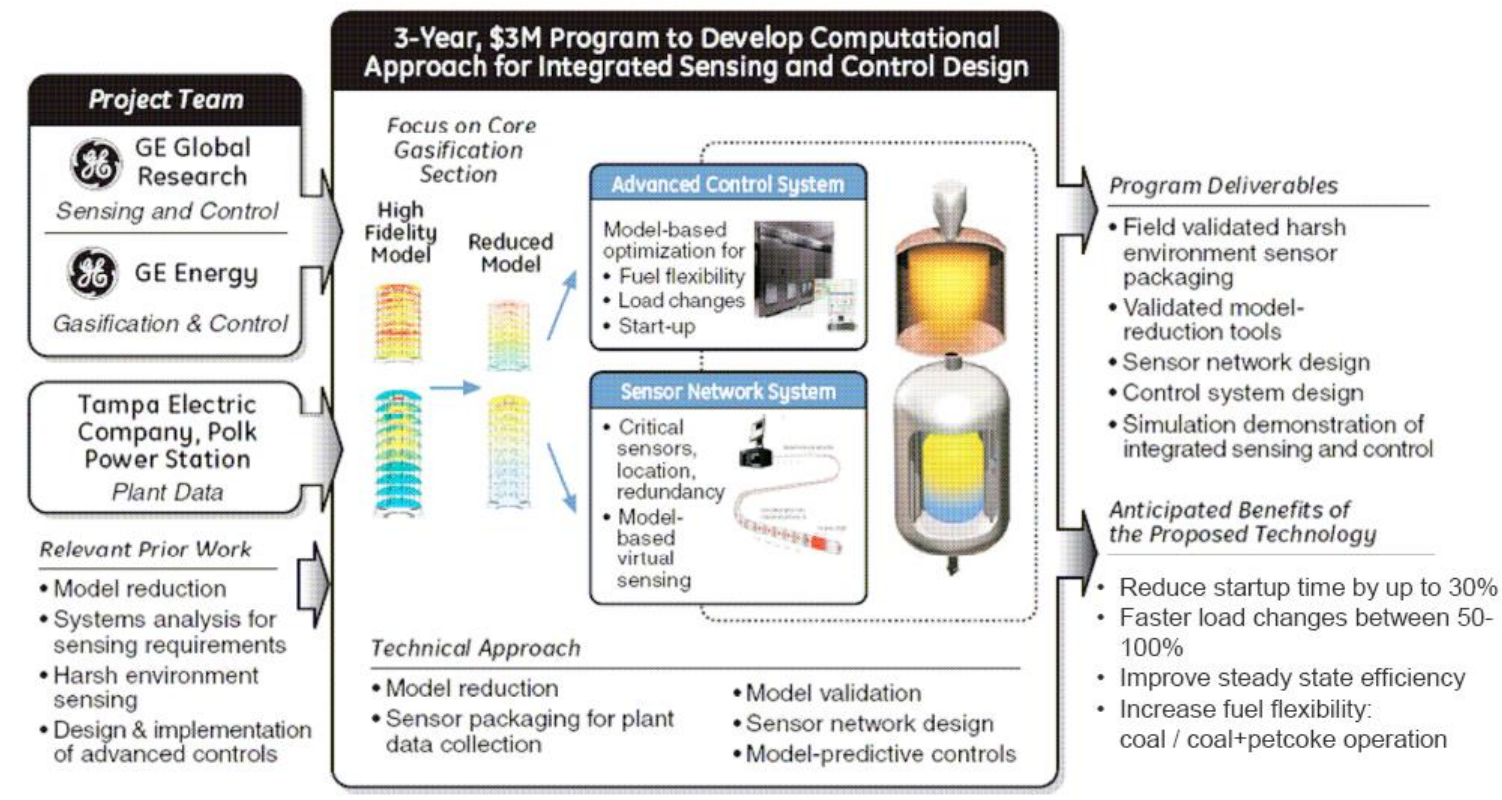

Figure 1: Program overview - team, scope and objectives.

In this three-year program, a systematic model-based approach was developed for the design of a comprehensive sensing system combining online sensors along with online model-based estimation, and a model-based multivariable controller that optimizes the operation of the gasification section at steady-state as well as through key transients like startup, turndown and fuel changes. The overall model-based sensing and controls design is shown in Figure 2. Initially, available models for different units of the gasification section (e.g. gasifier, radiant syngas cooler (RSC)) were combined in a common platform in Matlab/Simulink $®$ to obtain a comprehensive dynamic model of the gasification section. Also, specific sensing technologies were implemented in the IGCC plant at TECO Polk Power Station to obtain operation data to be used for the RSC model validation. The dynamic model of the gasification section was used in a systematic model-based analysis and design framework to design a comprehensive sensing and control system to improve the robustness and flexibility and optimize the operation of the gasification section for steady-state as well as transient operations, in particular, for startup, turndown and fuel changes. 


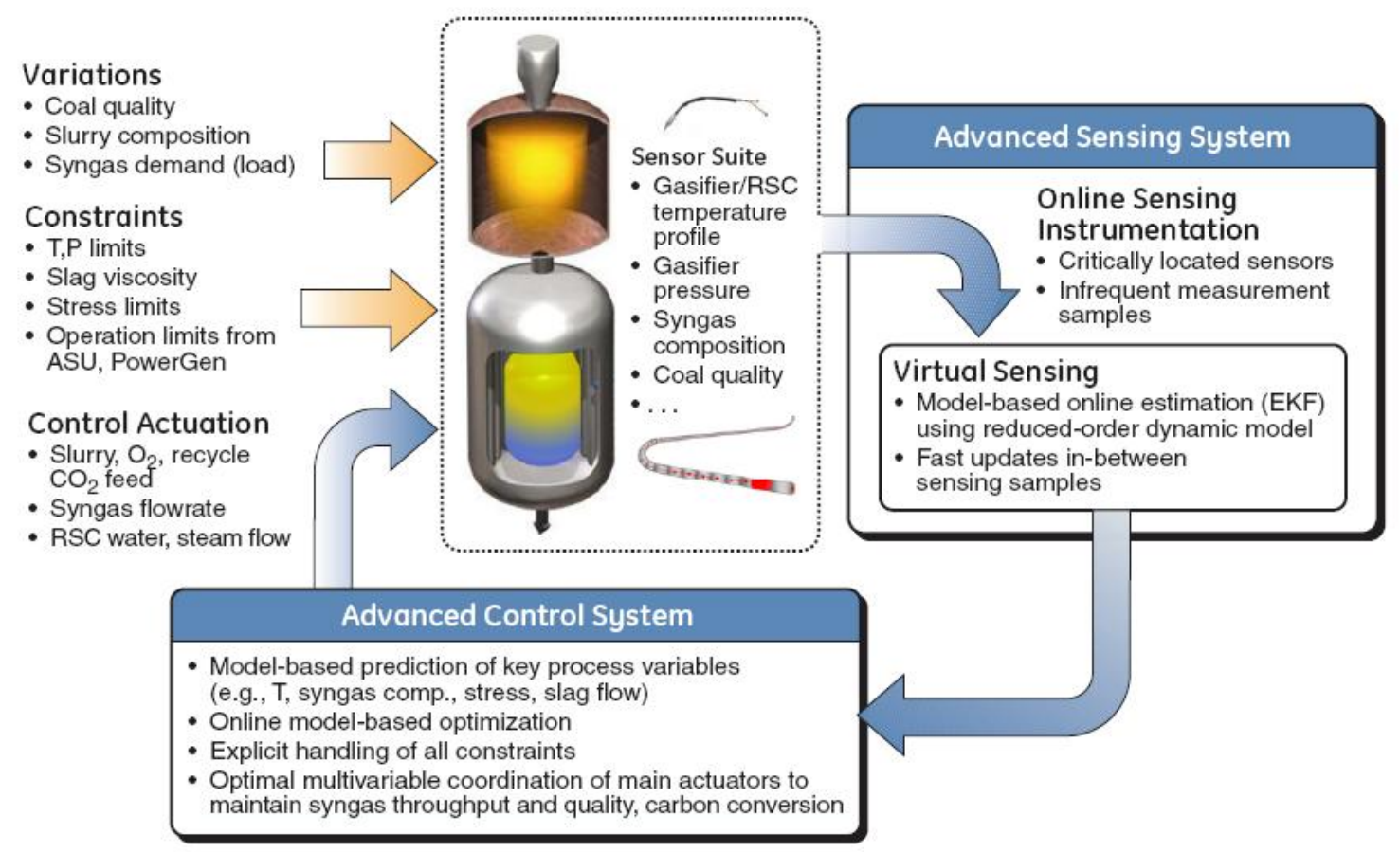

Figure 2: Integrated advanced model-based sensing and controls system for gasification section.

The overall three-year program had three main tasks:

1. Modeling, model reduction and model validation,

2. Sensing system design, and

3. Control system design.

In Task 1, available models for different units in the gasification section were combined in a common platform in Matlab/Simulink® to obtain the dynamic model for the overall gasification section, which was used for sensing and control system design in Tasks 2 and 3. Available component models for key process units were implemented in Matlab/Simulink $\AA$. Some of the existing models had only been used at steady state conditions and appropriates updates were made to allow transient simulation with these models. Furthermore, some of the models, e.g. gasifier and RSC, were of high order, i.e., they had a large number of internal states since they model spatial variation along the length of the gasifier and the RSC. Owing to the large dimension, these models are computationally expensive and not amenable to real-time transient simulations and modelbased analysis and design of sensing and control systems. Thus, these high-order models were simplified through model reduction techniques to obtain lower order models while maintaining high accuracy for control. A separate set of models was also implemented for startup pre-heating of the gasifier and RSC. These models are described in Section 4.1. Also, in Task 1, temperature and strain sensors were implemented in the RSC in the TECO Polk Power Station IGCC plant to obtain suitable operation data to be used for validation of the RSC model. A key common challenge for all the sensors was developing suitable packaging for the harsh environment in the RSC, and appropriate mechanical design to facilitate easy installation given the limited access inside the RSC. These sensors were installed in the TECO IGCC plant in the RSC in early 2009 and plant 
operation data was obtained successfully. The results from lab development and plant implementation are discussed in Section 4.2.

As mentioned earlier, the gasification section has a very harsh operating environment and as a result limited online sensors are available for monitoring and control, especially in the gasifier and RSC. A key objective of this program was to develop a comprehensive sensing solution, combining limited available online sensors with real-time online modelbased estimation, or "virtual" sensing for other key variables, e.g. gasifier temperature, and carbon conversion. Motivated by this, in Task 2 , the dynamic model of the gasification section from Task 1 was initially used to perform a systematic "observability" analysis. More specifically, a linear model-based Kalman filter analysis was performed to study the performance of model-based estimation for key unmeasured variables and its sensitivity to modeling and sensor errors. The sensitivity analysis allowed identifying the key sensor errors (bias and noise) and modeling errors (unknown/varying parameters), which were then included in an online nonlinear model-based estimation algorithm using an extended Kalman filter (EKF). Simulation studies were performed in the presence of modeling errors introduced through variations in model parameters, and noise and random biases in online sensors to verify the performance of the nonlinear model-based estimation system at steady-state as well as during transient operations. More details on this task are discussed in Section 5.

In Task 3, a model-based advanced controller was designed for the gasification section to coordinate the operation of the individual units in this section to optimize the overall section performance. In particular, a nonlinear model predictive controller (MPC) was implemented, initially assuming ideal measurement for all variables needed for feedback, to optimize the performance of the gasification section at steady state and during key transients like startup, turndown (i.e. load/throughput changes), and fuel changes.

Extensive MPC simulation studies were performed for startup, specifically gasifier and RSC pre-heating, and normal operation modes including turndown and fuel changes. These MPC simulations indicate opportunities for significant improvements in transient operation, reducing pre-heating and turndown transient times, and optimizing steady-state performance for minimized oxygen consumption. In the final Phase III of the program the EKF-based sensing system designed in Task 2 and MPC-based control system designed in Task 3 were coupled to achieve the overall integrated sensing and control system. The integrated system was updated and re-tuned to achieve stable and optimized operation, and its performance was studied through simulations with modeling and sensor errors. The results from this task are described in Section 6.

Finally, conclusions from the overall program and potential directions for application of the developed technology in an IGCC plant in a staged manner are summarized in Section 7.

\section{Task 1 - Modeling \& Sensor Implementation}

First-principles physics-based models allow capturing the process knowledge through rigorous mass and energy balances, and utilizing it systematically for improved online model-based sensing and controls. In the face of limited online sensors, first-principles models allow relating process variables across the entire system, and can thus be used as the basis for online model-based estimation or "virtual" sensing of key unmeasured 
process variables. Similarly, first-principles models capture the interaction between multiple control inputs and allow exploiting this multivariable interaction to optimize the process operation at steady state as well as during transients. With this motivation, the key objective of Task 1 was to obtain a suitable dynamic model of the gasification section in Matlab/Simulink® that can be used for real-time transient simulations and the design of advanced model-based sensing and control systems in Tasks 2 and 3. To this end, available component models for the individual process units in the gasification section were translated and implemented in Matlab/Simulink® to obtain the overall gasification section model for nominal operation. In the case of high-order models, specifically the gasifier model, model reduction was performed to obtain a low-order accurate model that allowed real-time simulation. In other cases, additional details were included to include key process dynamics. A separate set of models was also implemented for the startup preheating operation, specifically for RSC and gasifier pre-heating. These models are described in Section 4.1. Also, fiber optic temperature and strain sensors and thermocouple temperature sensors were installed in the radiant syngas cooler (RSC) in the IGCC plant at TECO Polk Power Station and plant operation data was obtained successfully. Details on the lab testing and design of the sensor packages, and the sensor implementation and the data collection are given in Section 4.2. Finally, Section 4.3 shows some validation results of the gasification section model modified to match plant operation data from the TECO IGCC plant at Polk power station.

\subsection{Modeling}

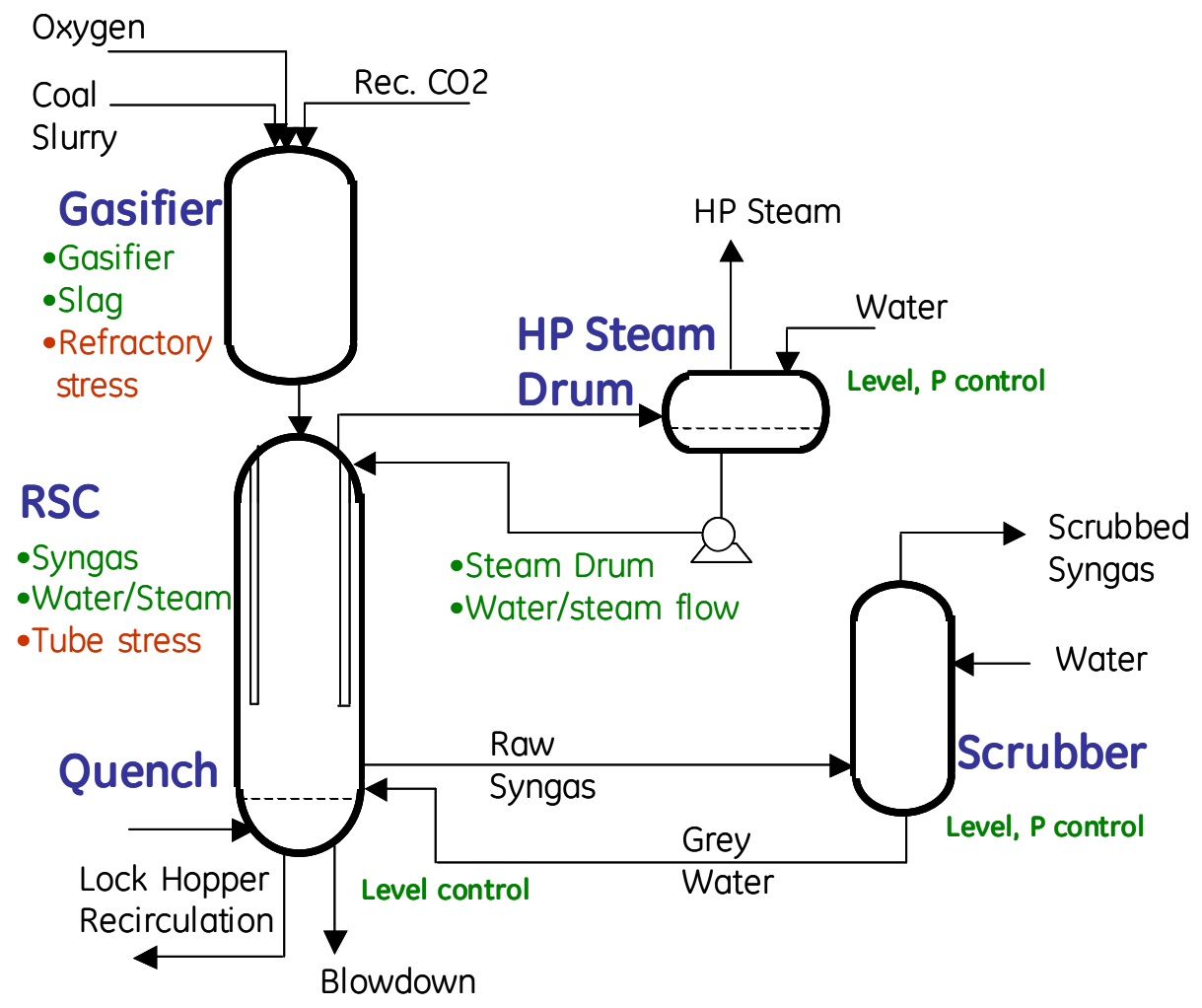

Figure 3: Gasification section. 
Figure 3 shows the overall scope of the gasification section considered in this program, starting from the coal slurry, recycle $\mathrm{CO}_{2}$ and oxygen feed to the gasifier, to the scrubbed syngas output and the high-pressure (HP) steam output. It should be mentioned, that the above process configuration is a general IGCC plant gasification section configuration, and is not specific to the TECO Polk Power Station IGCC plant. Also, to protect proprietary information, the model simulation and plant measurement data are presented in normalized units throughout the report. The key process units included in the gasification section are:

- Gasifier

- Radiant syngas cooler (RSC)

- Syngas quench and RSC sump

- Syngas scrubber

- RSC high pressure (HP) Steam Drum

The gasification section models have been developed for different phases of operation as shown in Figure 4. In particular, a model of the above units was implemented for nominal operation at high pressure, e.g. turndown between $50-100 \%$ load and fuel changes. This model was also extended to operate at lower pressures to cover the post-ignition pressure ramp-up phase of the startup, wherein the syngas and steam pressure are raised from pre-heating/ignition conditions to nominal operating conditions. Finally, another set of models was implemented for the pre-heating phase of the startup. In particular, the model for RSC and HP steam drum was modified to perform heat transfer in reverse direction, i.e. from the steam feed to HP steam drum and through heated water circulation to the RSC to pre-heat all the water tubes. This thermal model was coupled with a thermomechanical stress calculation model to calculate transient stress profiles that depend on the transient heating profile. In parallel, a similar model was implemented in Matlab ${ }^{\circ}+A N S Y S \AA$ for the pre-heating of the gasifier refractory, to describe the transient thermal profile in the refractory lining and the corresponding thermal stresses, which limit the pre-heating operation. These pre-heating and stress models were used in Task 3 to optimize the pre-heating sequence for faster startup.

\begin{tabular}{|c|c|c|c|}
\hline \multicolumn{3}{|c|}{ StartUp } & \multirow{2}{*}{$\begin{array}{l}\text { Nominal } \\
\text { Operation } \\
\text { (hi P) }\end{array}$} \\
\hline Pre-heating & Ignition & $\begin{array}{l}\text { Pressure } \\
\text { Ramp-Up }\end{array}$ & \\
\hline $\begin{array}{l}\text {-NG burners } \\
\text { for gasifier } \\
\text { refractory } \\
\text {-Steam } \\
\text { (<1000psi) for } \\
\text { RSC }\end{array}$ & $\begin{array}{l}\text { Coal } \\
\text { slurry+O2 } \\
\text { ( 50\% } \\
\text { flowrate) }\end{array}$ & $\begin{array}{l}\text { - Gasifier } P \\
\text { ramp to } \\
>600 \text { psi } \\
\text { - Drum P } \\
\text { ramp to } \\
\sim 2000 p s i\end{array}$ & $\begin{array}{l}\text {-Turndown } \\
\text { (50-100\%) } \\
\text {-Fuel changes } \\
\text { (coal + PC } \\
\text { blend) }\end{array}$ \\
\hline
\end{tabular}

Figure 4: Operation modes for gasification section and dynamic model.

For the nominal and pressure ramp-up portion of startup, all the above component models were implemented in Matlab/Simulink® and integrated to obtain the overall gasification section model. This model can be simulated transiently for nominal operation modes, e.g. load changes, coal/petcoke blend changes, as well as pressure changes during the 
pressure ramp-up phase of startup. Details on the component models and the overall gasification section nominal operation model are provided in the next few sections. The gasifier and RSC pre-heating models are described in the subsequent sections.

\subsubsection{Gasifier}

A 1-D (axial) gasifier model was available that had been validated against the TECO IGCC plant operation. This 1-D model was translated into Matlab/Simulink® and verified at baseload steady-state conditions against the original model. The model parameters were then updated to be consistent with the reference plant operating conditions. The model describes steady-state axial composition and temperature profiles along the length of the gasifier. Some of the key outputs of this model are the outlet syngas composition and temperature, ash flow and carbon conversion. Since the dynamics in the gasifier are quite fast compared to the transients like turndown and fuel changes, a steady-state model of the gasifier is adequate. However, due to the distributed model (PDE) and the high spatial resolution in the axial direction, the model was computationally expensive and not suited for real-time simulation or controls analysis/design. This necessitated a model reduction to obtain a lower-order model that could be used for real-time simulation and sensing \& control design, while preserving high accuracy.

The gasifier model reduction was pursued using the technique of Proper Orthogonal Decomposition (POD). To this end, initially the full-order model was simulated for a wide range of operating condition variations to encompass the desired operating envelope:

- throughput changes between 50-100\%,

- oxygen feed ratio changes between $-5 \%$ to $+7 \%$,

- coal slurry water content variation from nominal between $-10 \%$ to $+10 \%$, and

- recycle $\mathrm{CO}_{2}$ feed changes between $0-100 \%$,

- percent petcoke in coal-petcoke fuel blend between 0-50\%.

The results from these simulations (more than 400) were then used for the model reduction as highlighted in Figure 5. The original 1-D gasifier model describes the axial variation of ten gas-phase species flow rates along with three solid-phase components (ash, carbon and volatiles in coal) and liquid water (from slurry) arising from water boiling and devolatization of coal volatiles in the initial section and a set of thirteen heterogeneous (solid carbon + gas constituent) and homogeneous (gas-phase) reactions. The original model had altogether $\mathrm{N}=15$ gas/liquid/solid components, with corresponding flow rate profiles along the gasifier length for each simulation run. However, in the reduced model, only a reduced set of components was modeled through the dominant spatial modes. In particular, components like liquid water, coal volatiles and oxygen that are consumed/transformed in the immediate boundary layer at the gasifier inlet and have essentially zero flow rate through most of the gasifier are eliminated from the model. Similarly, ash, which doesn't participate in any reactions and is preserved (except for mass flux to the slag layer), is also modeled separately. Finally, the fact that there are reaction invariants, in particular the total elemental flow rates for $\mathrm{C}, \mathrm{H}, \mathrm{N}, \mathrm{O}$ and $\mathrm{S}$ are constant along the gasifier length was used to further eliminate the number of species to be modeled directly through the dominant spatial modes. In particular, a Q-R factorization of the reaction stoichiometric matrix was used to identify $(C=6)$ gas-phase components that are most aligned with orthogonal directions to the reaction invariants (i.e. total $\mathrm{C}, \mathrm{H}, \mathrm{N}, \mathrm{O}$, $S$ element flow rates) - this ensures that the $\mathrm{C}=6$ components are independent and can 
then be used to reconstruct the remaining 5 components from the total element balance without any ill-conditioning, i.e. the $\mathrm{C}=6$ components and the five elements $\mathrm{C}, \mathrm{H}, \mathrm{N}, \mathrm{O}, \mathrm{S}$, together comprise a well-conditioned coordinate change. This way, instead of identifying dominant spatial modes for $\mathrm{N}=15$ components, a lower number of species $\mathrm{C}=6$ are used to identify the dominant spatial modes.

\section{- Original Model}

$$
\begin{gathered}
\text { Material Balance: } \frac{\partial F_{i}}{\partial x}=M W_{i} V \alpha S_{i} R(F, T) \quad \text { (for each species } i \text { ) } \\
\text { Energy Balance: } \quad 0=\left(E_{\text {feed }}-E_{\text {exit }}\right) / E_{\text {feed }}, \quad E=f(F, T) \\
\qquad \begin{array}{ccc}
\mathrm{R}_{\mathrm{N}} \text { Simulations for varying operating conditions } \\
\mathbf{F}_{1}=\left[\begin{array}{ccc}
F_{1}^{R 1} & \cdots & F_{1}^{R_{N}} \\
F_{C}^{R 1} & \cdots & \vdots \\
& \cdots & F_{C}^{R_{N}}
\end{array}\right] \\
\text { Identify M dominant modes using SVD }
\end{array}
\end{gathered}
$$

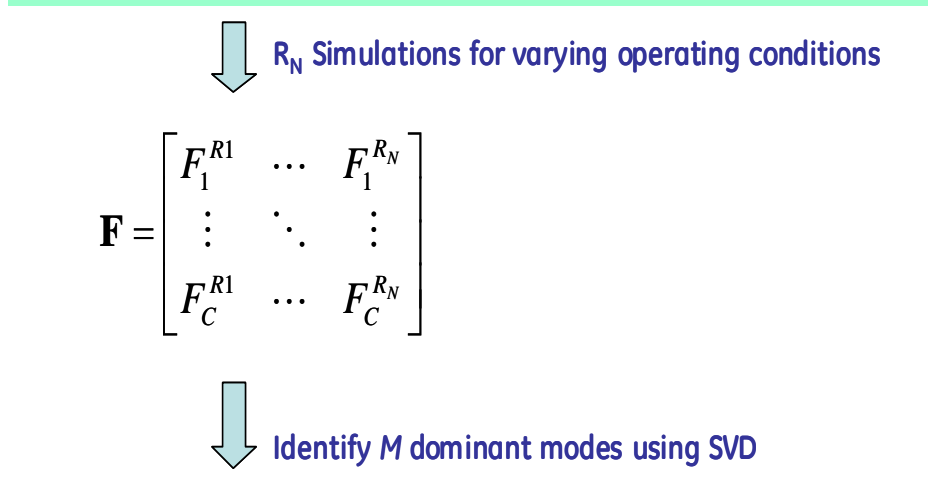

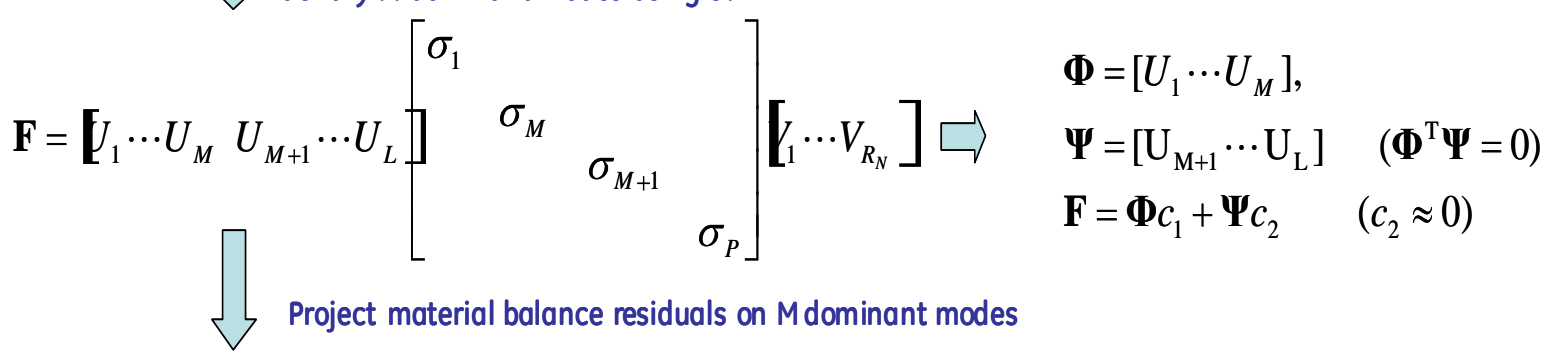

\section{- Reduced Model}

$$
\begin{array}{ll}
\text { Material Balance: } & 0=\boldsymbol{\Phi}^{\mathrm{T}}\left\{\boldsymbol{\Phi} c_{1}-M W_{i} V \alpha S_{i} R\left(\boldsymbol{\Phi} c_{1}, T\right)\right\} \\
\text { Energy Balance: } & 0=\left(E_{\text {feed }}-E_{\text {exit }}\right) / E_{\text {feed }}, \quad E=f\left(\boldsymbol{\Phi} c_{1}, T\right)
\end{array}
$$

\section{Figure 5: Model reduction approach for 1-D gasifier model.}

For the $\mathrm{C}=6$ species, the flow rate profile from each of the $\mathrm{R}_{\mathrm{N}}$ simulation runs were stacked in columns of a matrix and a SVD performed to identify the desired number of dominant spatial modes. To improve the performance of the SVD and dominant mode identification, two key transformations on the raw flow rate data were employed. First, for the species with very small flow rates (e.g. $\mathrm{COS}, \mathrm{NH}_{3}$ ) the flow rates were transformed using a log transform. This does two things (i) it converts small values to significant values that are then captured with appropriate weights in the identified dominant modes, and (ii) it provides a natural barrier in the reduced model from calculating negative flow rates due to small numerical errors - any negative flow rates would be physically meaningless and render the reaction kinetics expressions unusable. Second, the flow rates (or log of flow rates for species with low concentrations) were obtained in terms of deviations from the nominal (baseload) operating condition, and normalized using constant scale factors. The 
SVD analysis was used to identify $M=15$ dominant modes, which are shown in Figure 6 for the $\mathrm{C}=6$ species modeled directly as combinations of the dominant spatial modes. The number of modes to retain was identified by projecting the full-order model simulation results along these modes and evaluating the residual error. Thus, finally a low-order $(\mathrm{M}+1)$ model was derived by projecting the original PDE model equations along these dominant modes.
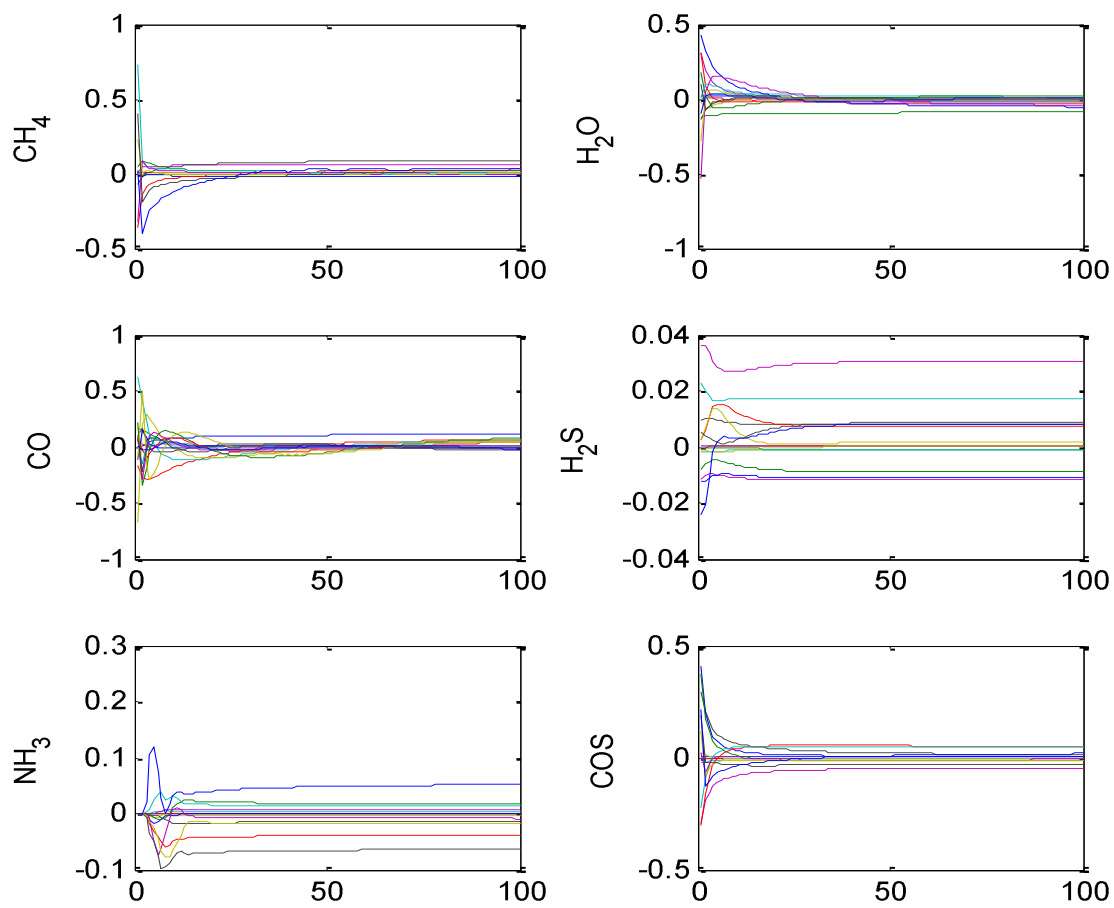

Figure 6: Dominant spatial modes (first 15) for the $C=6$ species modeled as combinations of these spatial modes in the reduced-order model.
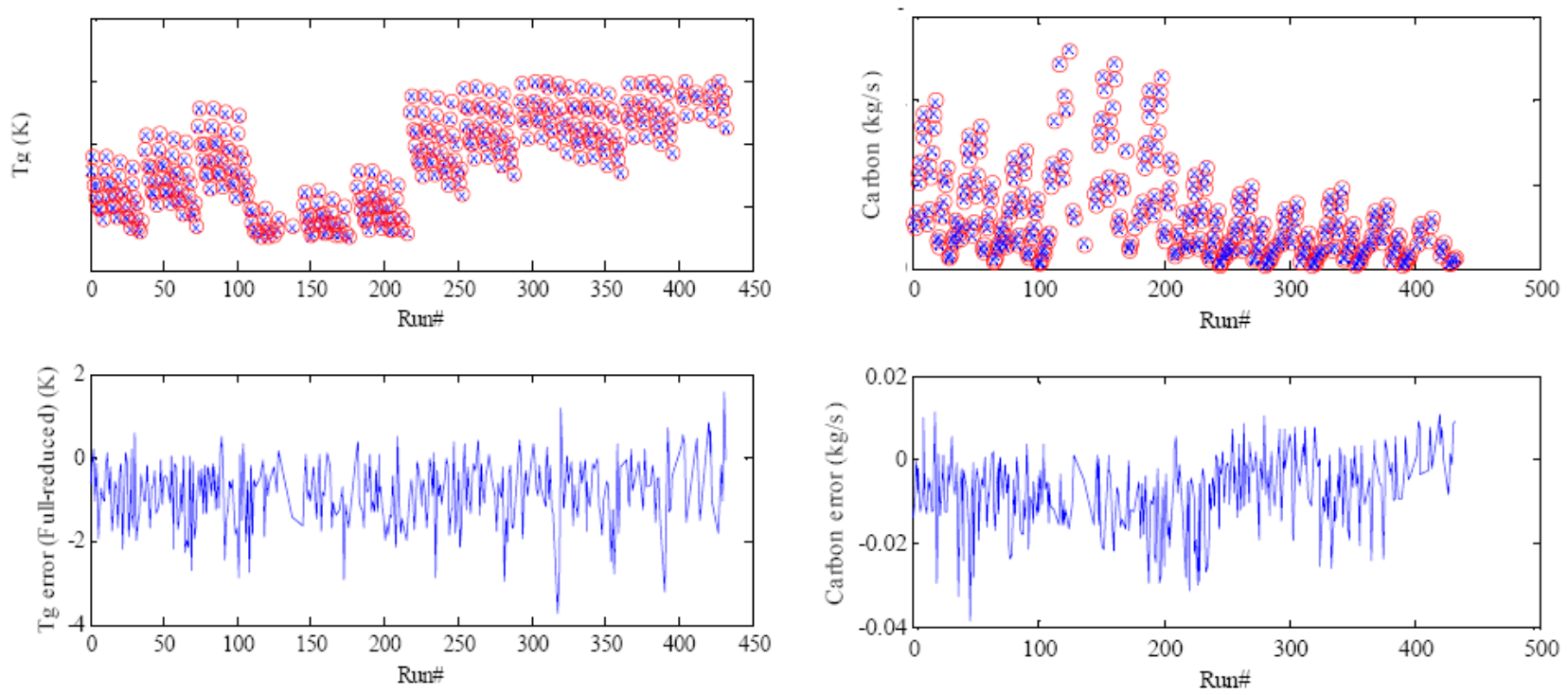

Figure 7: Comparison of full-order (blue $\mathrm{x}$ ) and reduced-order (red circles) simulation results for gasifier exit temperature and unconverted carbon flow rate, and corresponding errors between full- and reduced-order models. 
The reduced-order model runs more than $1000 x$ faster than the original full-order model while maintaining very high accuracy - the faster simulation of the reduced-order model was critical for subsequent real-time simulation and sensing \& controls design. The reduced-order model results were compared against the original model for a wide range of variations in operating conditions listed above and the error between the two models was approximately $2 \mathrm{~K}$ for syngas temperature and less than $0.2-0.4 \%$ in syngas composition. The comparison between the full- and reduced-order models for gasifier exit temperature, unconverted carbon flow rate and some syngas component compositions at gasifier exit are shown in Figure 7 and Figure 8. The original model took a few minutes (ranging from 10-30 minutes) to converge at a steady state, which is too slow for a real-time transient simulation of the transients like turndown and fuel changes. The reduced-order model converges to steady state within a fraction of a second and allowed simulating transiently for changes in operating conditions, e.g., throughput changes, oxygen feed changes, coal blend changes, and could be used for sensing and controls design.

포
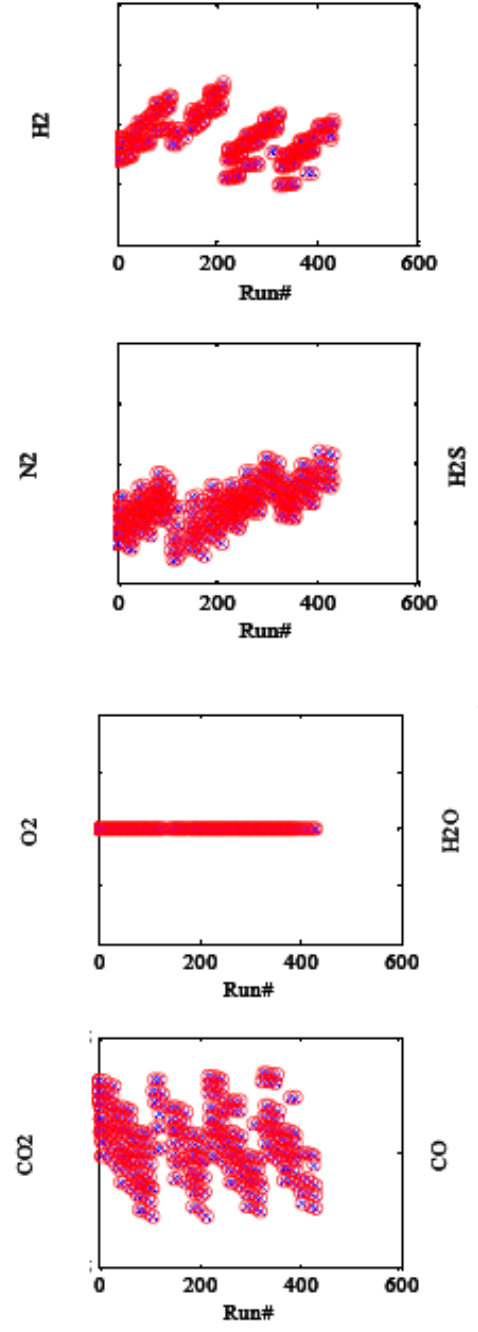
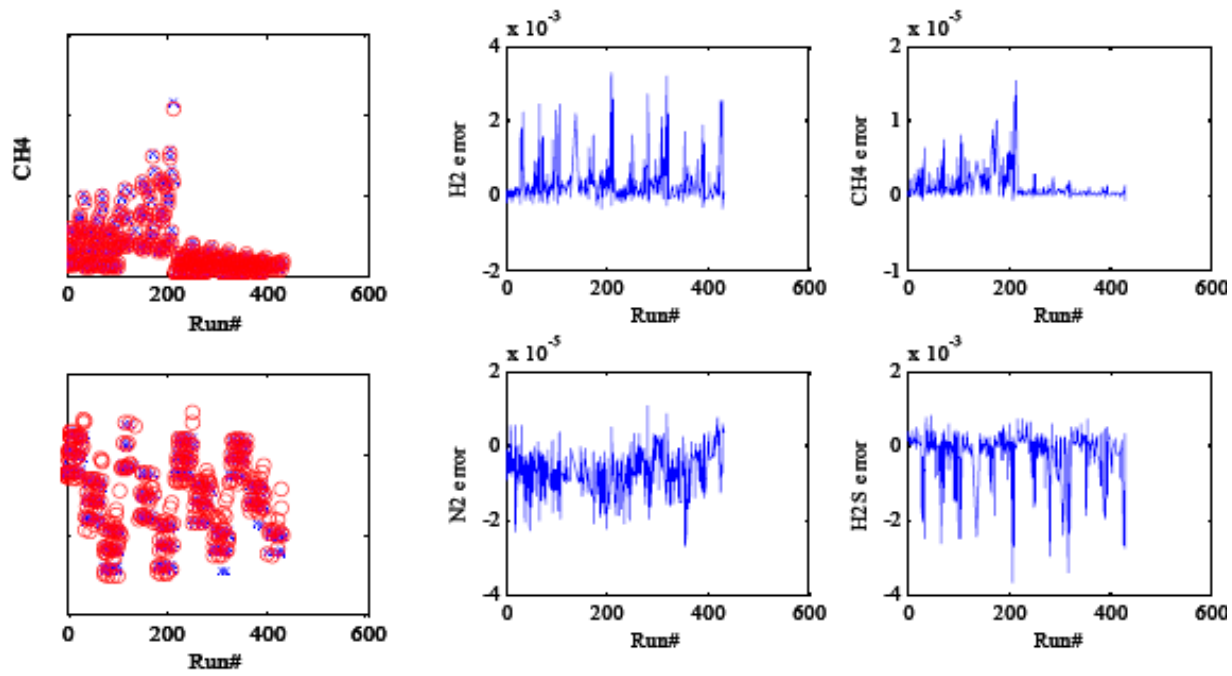
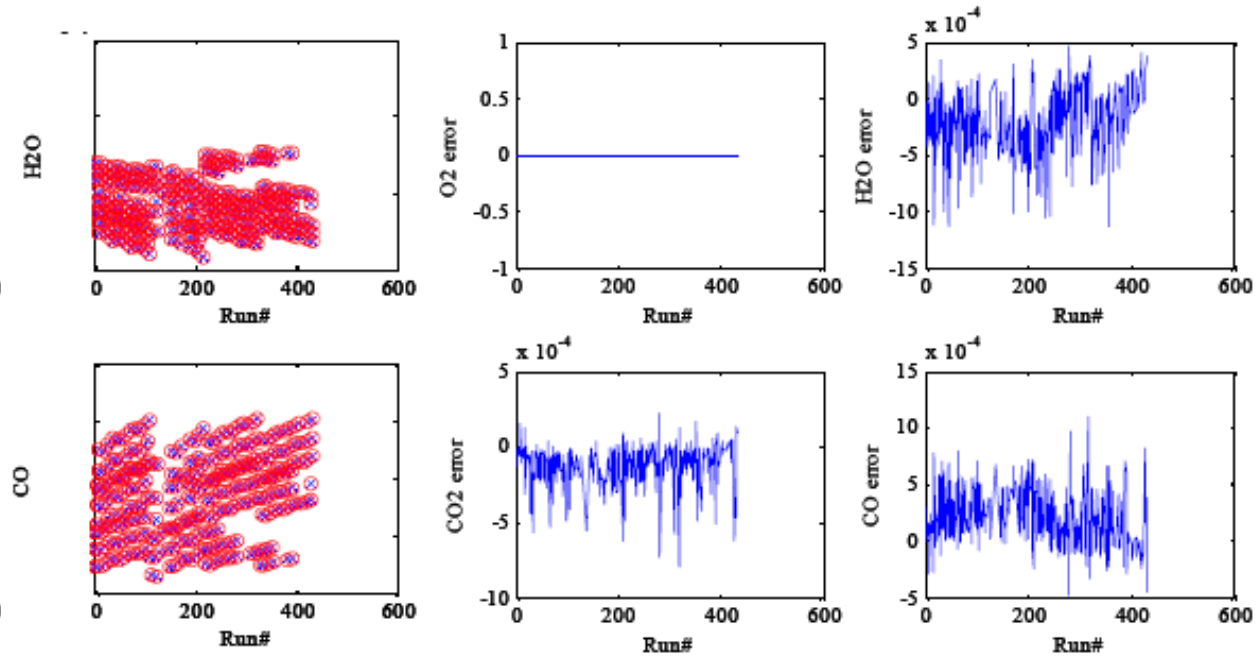

Figure 8: Comparison of full-order (blue $\mathrm{x}$ ) and reduced-order (red circles) simulation results for some syngas component compositions at gasifier exit, and corresponding errors between full- and reduced-order models. 
The performance of the reduced order model was also compared against the original fullorder model for low-pressure conditions during startup post-ignition, pressure ramp-up phase using the same dominant spatial modes obtained for nominal operation. As expected, the error increased slightly. For instance, the errors in exit temperatures increased from $\sim 2 \mathrm{~K}$ to $\sim 8-10 \mathrm{~K}$, and errors in exit unconverted carbon flow rate increased from $\sim 0.02 \mathrm{~kg} / \mathrm{s}$ to about $0.25 \mathrm{~kg} / \mathrm{s}$, which are still quite small compared to desired model accuracy, especially for the relatively short post-ignition pressure ramp-up phase of startup operation. So the reduced-order gasifier model was also used for simulation and controls design for the pressure ramp-up phase.

\subsubsection{Slag Flow Model}

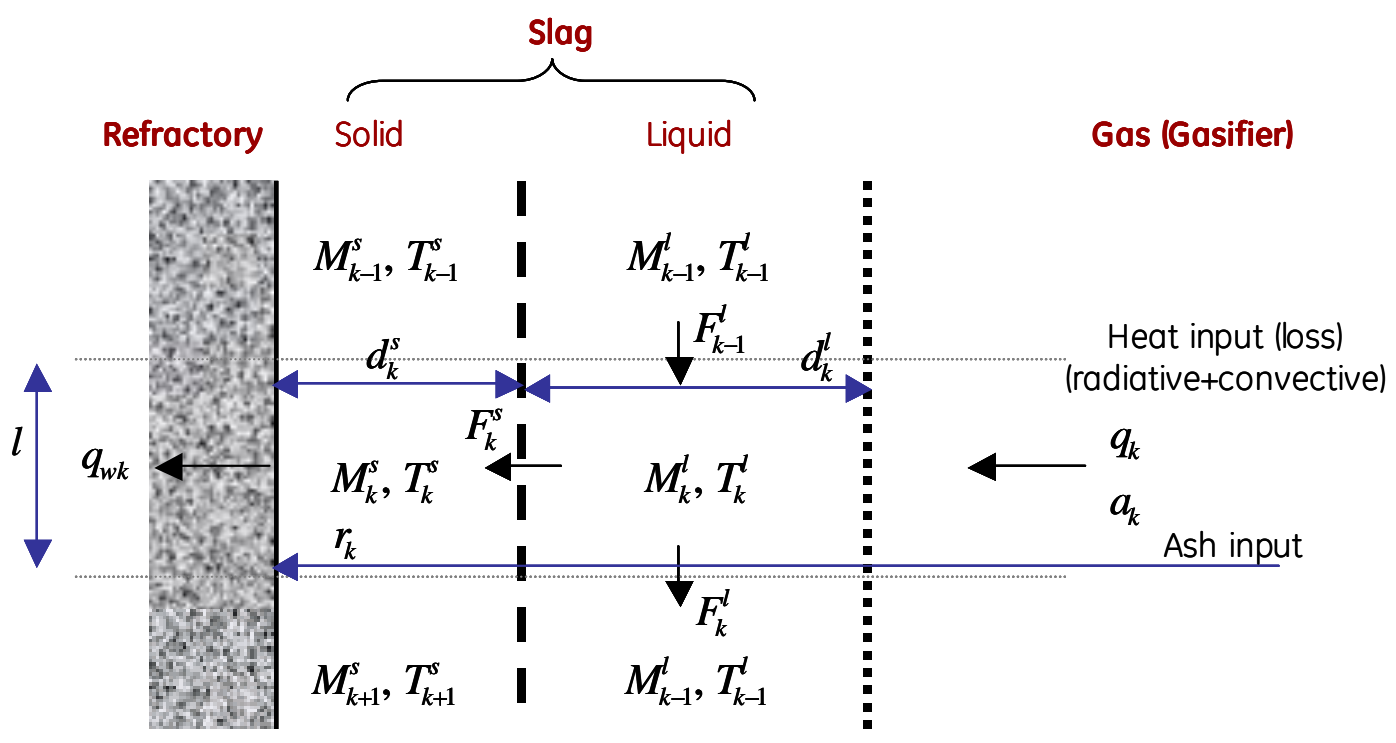

Figure 9: Gasifier slag flow model structure.

In addition to the gasifier model, a model for slag flow on the gasifier refractory wall was also implemented as depicted in Figure 9. The model for slag flow takes as inputs, the ash and heat flux from the gasifier along its length and calculates the temperature profile as well as profiles for mass holdups (and thus thickness) of a solid and liquid slag layer along the gasifier length based on slag viscosity and resulting flow rate obtained as a function of ash composition and local temperature. The original slag flow model was very detailed with high spatial resolution in the axial as well as the radial direction and modeled only steady-state conditions. This model was translated to Matlab/Simulink $\AA$, using a coarser spatial resolution and adding dynamics to the material and energy balances. This model was then interfaced with the reduced-order gasifier model to provide transient evolution of the liquid and solid slag thickness along the gasifier length based on transient variations in the gasifier operation. 


\subsubsection{Radiant Syngas Cooler (RSC) and High Pressure (HP) Steam Drum}

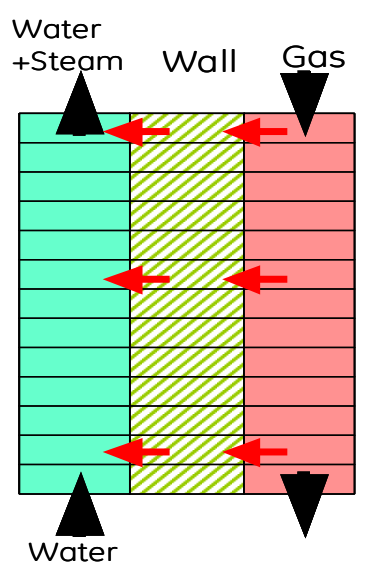

Figure 10: 1-D (axial) RSC heat transfer model for counter-current flow of hot syngas and water/steam and heat exchange.

A 1-D (axial) RSC transient model describing the transient energy balances between the hot syngas and water/steam mixture flowing in counter-current direction was available. This model was converted into Matlab/Simulink®. This model takes as input the exit streams from the gasifier and calculates the convective and radiative heat flux along the length of the RSC into the riser tubes and the water flowing inside in counter-current direction to generate steam, as shown in Figure 10. The translated model included dynamic energy balances for the syngas, tube metal and the water/steam mixture in the tubes to obtain the transient variation in the hot syngas temperature, the tube metal temperature and the steam fraction in the water stream along the length of the RSC. This model in Matlab/Simulink® was verified against the original model. However, this model had quite stiff dynamics with the metal temperature states having much faster dynamics, which necessitated the use of a variable step-size stiff solver in Simulink. While this is adequate for a pure simulation environment, it is desirable to use a fixed step-size solver when coupling with discrete-time controls as needed in Tasks 2 \& 3. To this end, a quasisteady-state approximation of the fast metal temperature states was employed to eliminate stiffness and enable fast simulations with a fixed step-size solver, while still preserving very high accuracy. This 1-D (axial) RSC model was also compared with a more detailed 3-D CFD model to derive detailed profiles for heat transfer calculations along the length and obtain a good match between the 1-D and 3-D models for the average axial temperature profile and heat flux profile along the RSC length. Typical steady-state profiles for steam mass fraction (void fraction), water temperature and syngas temperature along the RSC length are shown in Figure 11 for clean RSC at baseload and half load conditions as well as for fully fouled RSC at baseload conditions. 

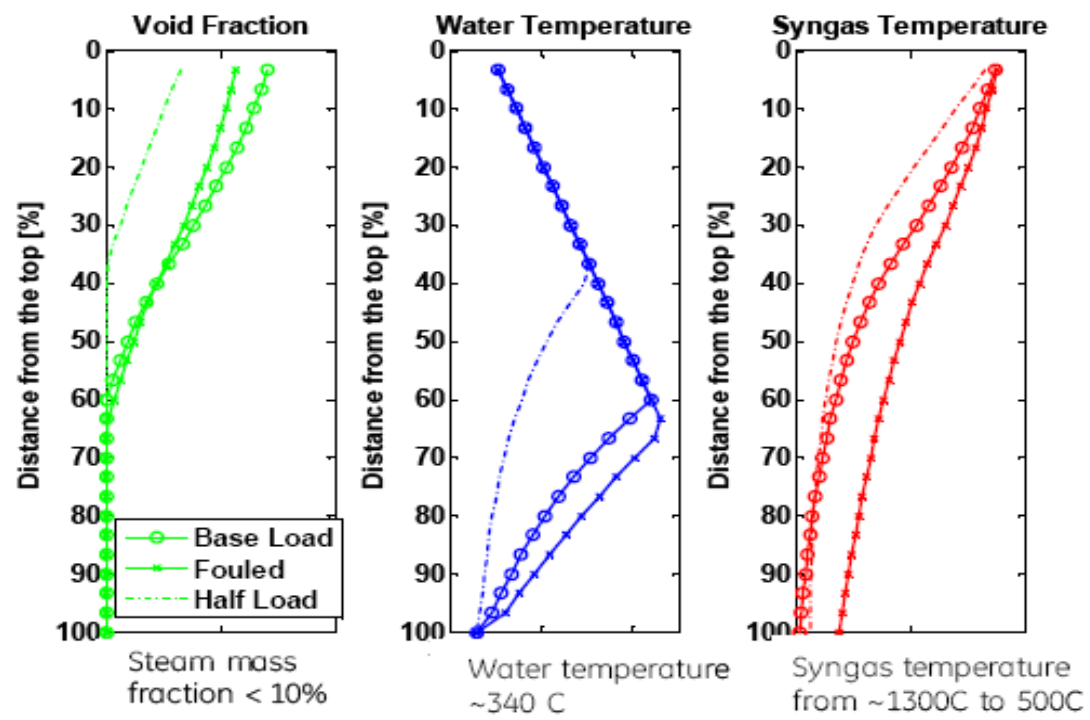

Figure 11: Steady-state profiles for steam mass fraction, water and syngas temperature in RSC for base-load and half-load conditions in clean RSC and base-load conditions with fully fouled RSC.

In addition to the transient heat balance in the RSC, the RSC model was updated to calculate updated syngas composition profiles along the RSC length due to gas phase reactions varying with changes in the syngas temperature. In particular, the water gas shift reaction and methane steam reforming (or methanation reaction in reverse direction) were included to capture the variation in syngas composition along the RSC length. While ideally, the energy balance and the composition changes due to these reactions should be coupled, they are run in a sequential manner since the net heat of reaction for these reactions with small composition changes along the RSC length are very small and can be ignored compared to overall RSC heat duty.

The RSC model was coupled with a high-pressure (HP) steam drum model and a flow model for calculating the water/steam circulation flow rate between the drum and the riser/downcomer in the RSC. The HP steam drum model was translated from another modeling platform to Matlab/Simulink ${ }^{\circledR}$ and it describes the dynamic behavior of liquid and steam holdups and enthalpy as well as drum metal temperature. While this model is sufficient for nominal operating conditions after startup, i.e. at high steam pressure in the drum, additional features had to be added to describe important transients during the pressure ramp-up phase in startup. In particular, modifications to incorporate steam holdup under the water level in the drum as shown in Figure 12, and dynamic material balances in the RSC riser water/steam holdups were added - these features are important to describe the transient "swell" characteristics in the liquid level that are very significant at low pressures during startup. 


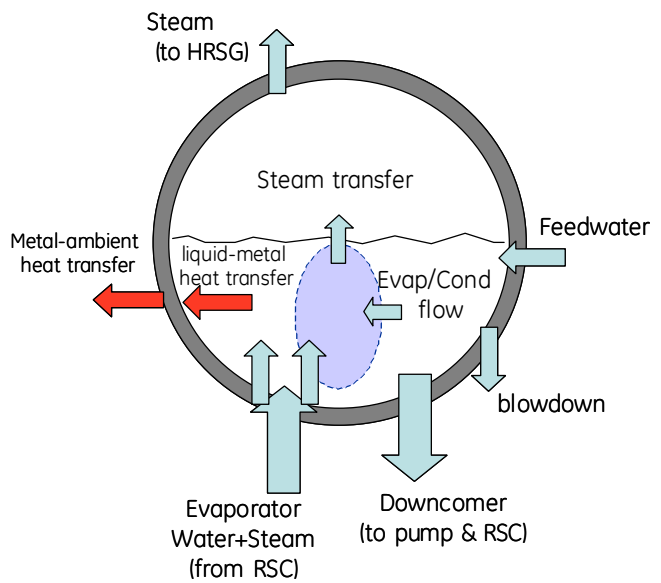

Figure 12: RSC HP steam drum model structure with steam holdup under the water level.

Figure 13 shows an example simulation run for the update RSC, HP drum model for a $5 \%$ drop in drum pressure starting from steady-state conditions at nominal operating pressure. The right middle and bottom plots show the variation in the water flow rate along the RSC tube length as well as the variation in the volume fraction of steam holdup below water level in the drum during the transient. These characteristics result in a transient "surge" in the level as shown in the plots in bottom left despite using a PI level controller. Changes in drum level during pressure transients, especially at lower pressure during startup, are important to capture since the drum level has fairly tight operability constraint limits.
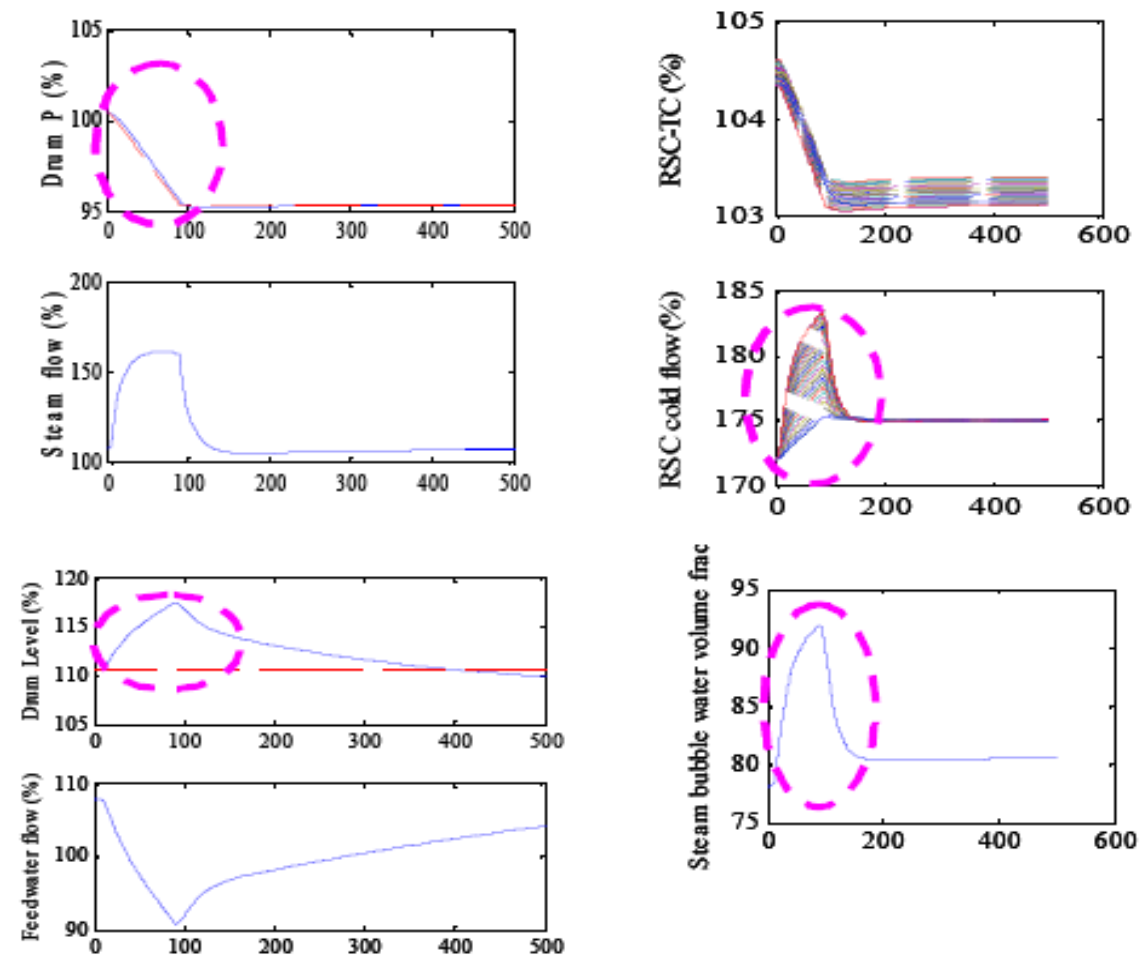

Figure 13: Simulation run with 5\% drop in HP steam drum pressure showing transient drum level surge due to flow rate variation in RSC tubes and variation in steam volume fraction under water level during the transient. 


\subsubsection{RSC Quench/Sump and Scrubber}

A model of the RSC syngas quench and the water/slag flow through the sump was translated into Matlab/Simulink®. This model takes as input the syngas and ash/slag streams from the RSC outlet, and calculates the outlet syngas stream flow rate, composition and enthalpy, especially for change in water vapor content and temperature using a 1-stage flash non-ideal vapor liquid equilibrium (VLE). This model was also validated against the original Aspen dynamics model. Similarly, a model of the scrubber was translated into Matlab/Simulink ${ }^{\circ}$. This model takes the syngas output from the quench as input and calculates the outlet syngas composition and temperature.

The above sections described the individual component models used for the nominal \& pressure ramp-up phase of the gasification section operation. The next two sections describe the models for RSC and gasifier refractory pre-heating during plant startup.

\subsubsection{RSC Pre-heating}

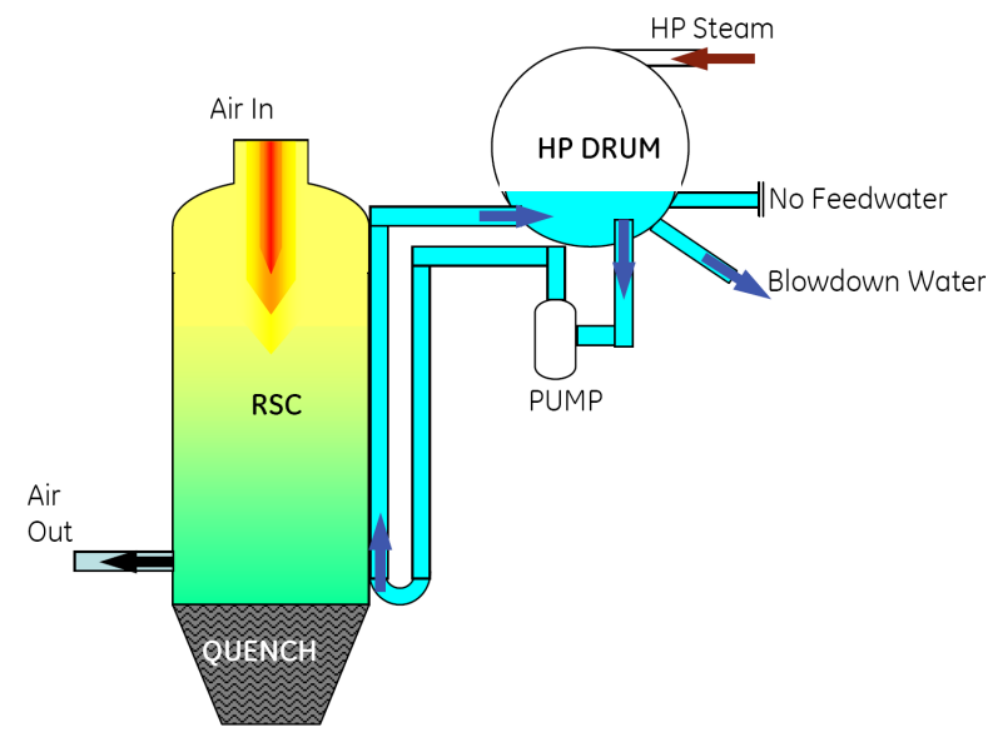

Figure 14: RSC pre-heating with steam.

Figure 14 shows the schematic representation of the RSC and HP steam drum operation for startup pre-heating. Starting from ambient temperature and pressure conditions, steam is supplied into the HP drum and gradually the pressure in the HP drum and the recirculating water temperature is raised, thereby heating the tubes and the shell in the RSC. The water level in the HP drum is maintained through blowdown of condensate. A transient thermal model for the pre-heating of the RSC was implemented in Matlab/Simulink $\circledast$. Figure 15 shows an example set of transient temperature profiles from RSC pre-heating in the TECO IGCC plant for the RSC tubes and the shell (solid red/green plots), and the corresponding plots obtained from the transient thermal model (dashed $\mathrm{red} / \mathrm{green}$ plots). The dashed black plot shows the saturation temperature of the water corresponding to the drum pressure. The tubes are directly heated by the circulating hot water and heat up faster. On the other hand, the shell is indirectly heated through heat transfer from the tubes, and also has high thermal capacity, causing it to heat up slower, thereby creating thermal differences and stresses. These thermal differences and stresses 
impose a limitation on the pre-heating operation of the RSC. Clearly, the model outputs for the transient temperature profiles of the tubes and the shell match very well with the measured temperatures during RSC pre-heating. The temperatures for the tubes and the vessel shell are used to calculate the different thermal expansions and corresponding changes in the stresses in the RSC piping structure. This model is used in Task 3 to optimize the pre-heating transient in order to minimize the total pre-heating time needed to reach the final desired temperature while ensuring that the transient temperature and stresses are always within acceptable limits.

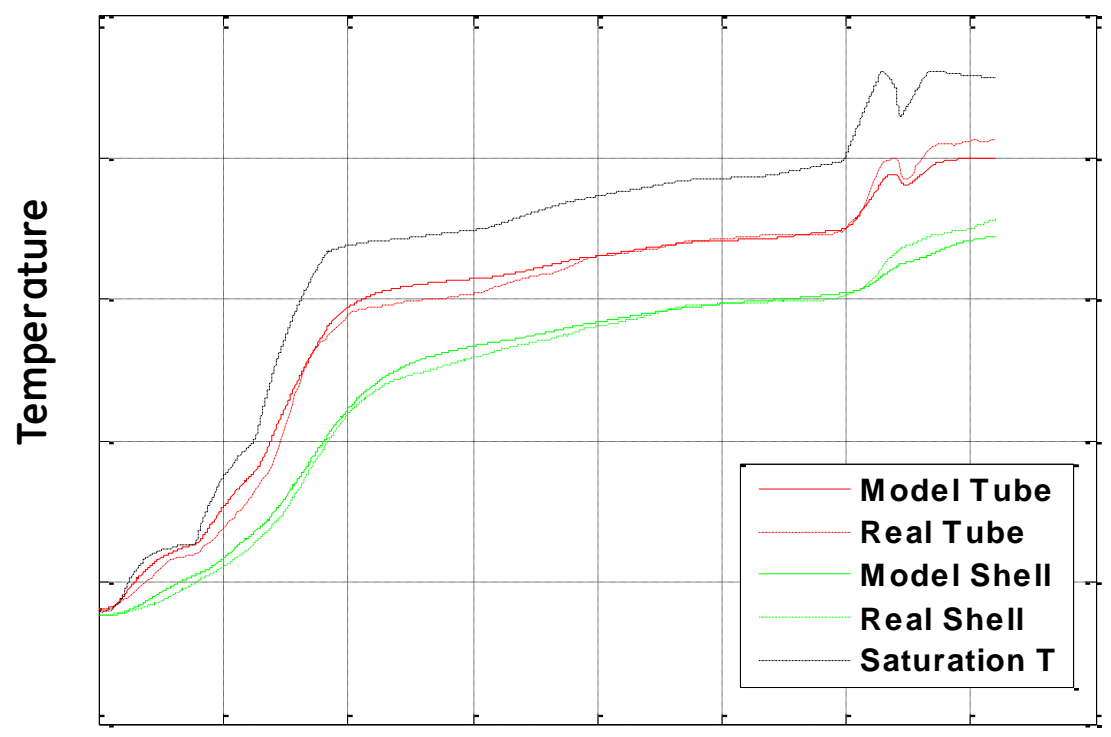

Time

Figure 15: Pre-heating temperature profiles in RSC - comparison of model outputs and TECO IGCC plant startup operation data.

\subsubsection{Gasifier Pre-heating}

Analogous to the RSC pre-heating, the gasifier refractory lining is also pre-heated by using auxiliary burners in the gasifier. The direct pre-heating of the inside of the refractory lining, and the corresponding heat transfer through the refractory lining and the outside metal shell leads to a temperature gradient in the refractory lining, and thus, buildup of tensile and compressive stresses. The transient thermal model is obtained as 1-D (radial direction) conductive heat transfer from the inner surface of the innermost refractory layer, through the multiple refractory layers and the metal shell and subsequent convective heat loss to the ambient environment outside. The temperature is symmetric in the azimuth direction, while the variation in the axial direction is small and ignored. More specifically, the transient radial heat transfer equation

\section{Eq 1}

$$
\frac{\partial^{2} T}{\partial r^{2}}+\frac{\partial T}{r \partial r}=\frac{\rho c_{p}}{K} \frac{\partial T}{\partial t}
$$

is discretized using finite difference with a specified set of boundary conditions - inner surface temperature (at $r=r_{0}$ ) and outside ambient air temperature for heat loss through convective heat transfer from the metal shell. Also, temperature dependent material 
properties (e.g. thermal conductivity and specific heat capacity) are used for respective refractory layers and the metal shell.
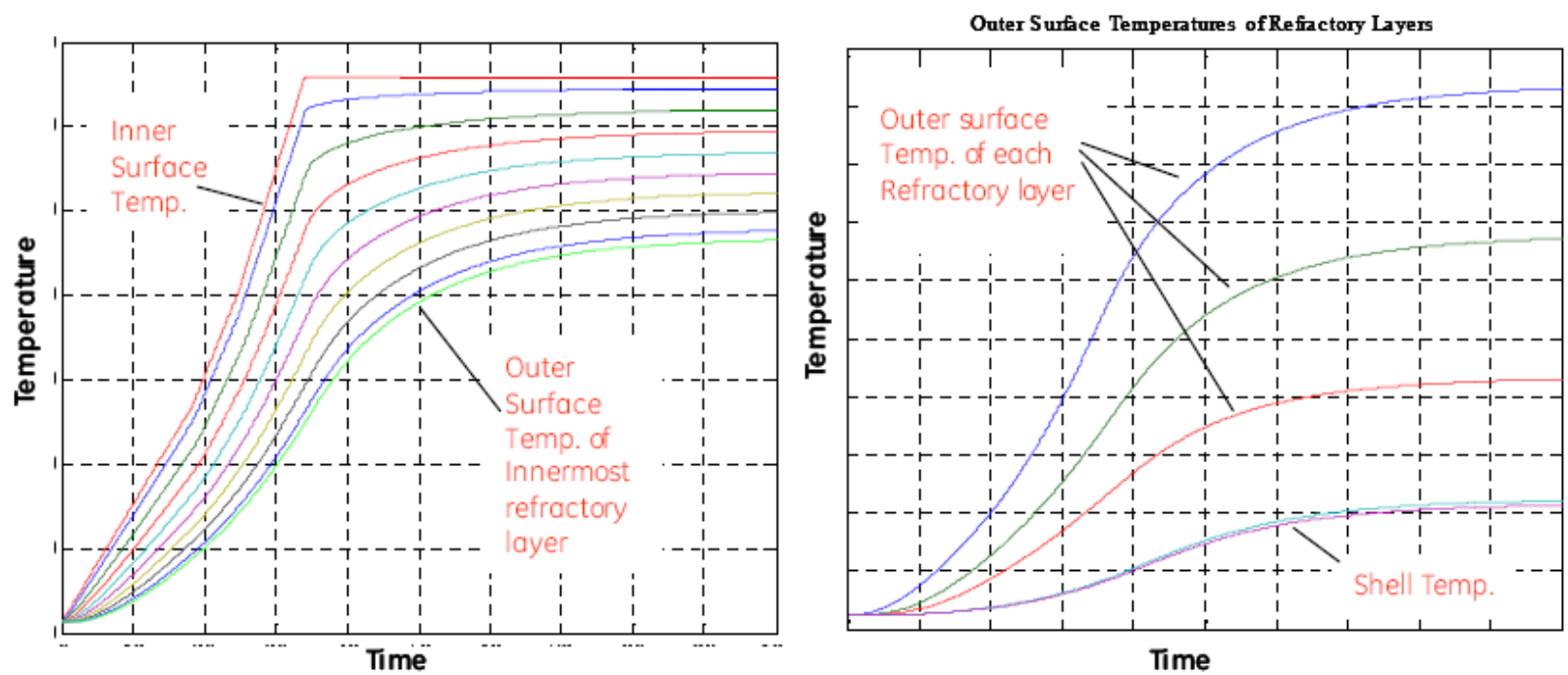

Figure 16: Transient temperature profiles in the gasifier refractory layers and metal shell during pre-heating obtained by transient thermal model.

Figure 16 shows the relative transient temperature profiles across the multiple refractory layers and the outside shell for the baseline pre-heating temperature profile on the inner (hot) surface of the innermost refractory layer. The radial temperature gradient in the refractory layers, is a function of the pre-heating temperature profile, and leads to corresponding tensile and compressive stresses. The thermal model is implemented in Matlab/Simulink $\circledast$ to calculate the transient thermal profile across the refractory layers and the metal shell. Owing to the complicated geometry of the refractory layers, an ANSYS ${ }^{8}$ model was implemented to calculate the tensile and compressive stresses in the innermost refractory layer (with the maximum temperature gradients and thus with maximum stresses) for a given temperature profile. This ANSYS $\circledast$ model was coupled to the Matlab/Simulink® thermal model to obtain the overall transient thermal and stress model for the gasifier pre-heating. Figure 17 shows the transient tensile and compressive stresses in the innermost refractory layer for the baseline pre-heating sequence for a design that allows contact between the refractory bricks and compressive hoop stresses to build up during pre-heating. In particular, initially the hoop stresses are very small due to gaps between the refractory bricks at low temperatures. However, with heating and expansion these gaps close and thereafter the compressive hoop stress builds up while the tensile stress decreases. This simulation run shows that the compressive stress reaches approximately $70 \%$ of the compressive strength and the tensile stress reaches a peak value of approximately $40 \%$ of tensile strength at the point when the gaps between the refractory bricks close up. Clearly, the temperature profile across the refractory layers and the corresponding thermal growths and tensile/compressive stresses are a function of the pre-heating temperature profile followed for the inner (hot) surface. This pre-heating profile is optimized under Task 3 to minimize the total pre-heating time needed to reach the final desired refractory temperature while not exceeding the peak compressive and tensile stresses observed for the baseline pre-heating profile. 


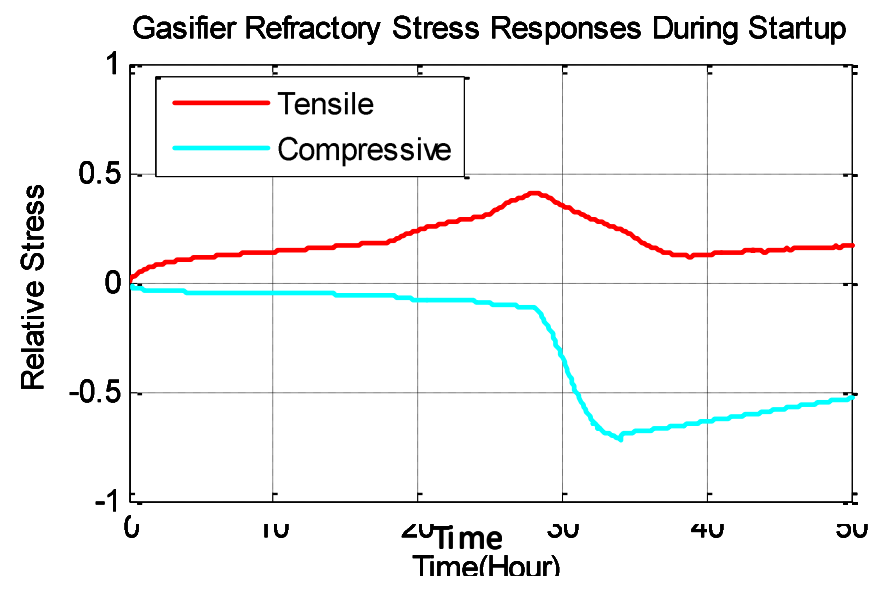

Figure 17: Transient profiles for tensile and compressive stresses as fraction of respective strengths in the innermost refractory layer obtained by the ANSYS $₫$ model.

\subsection{Sensor Implementation in TECO IGCC Plant}

In this sub-task (Task 1.5), the objective was to design and implement sensors in the RSC in the TECO Polk Power Station IGCC plant to obtain plant operation data that could be used to validate the models for RSC. Initially, three potential candidates for sensor implementation were identified for installation in the RSC:

- Radial temperature profile measurement inside RSC at levels 7 and 10 using a combination of thermocouples and fiber optic sensors. The key challenge for this option was survivability of the sensor and packaging for the harsh environment temperatures up to 2000 or more was expected.

- Axial temperature profile measurement inside RSC between levels 7 and 10 and using fiber optic sensors and thermocouples. In addition to harsh environment and survivability challenges, an additional challenge was the mechanical design and installation of the sensing package due to the very limited access inside the RSC.

- Strain measurement outside the hot gas path using fiber optic strain sensors. Key challenges for this were sensor bonding to the strain surface and sensor survivability at temperatures up to $700 \mathrm{~F}$ and corresponding large thermal strains $(\sim 5000-7000 \mu \varepsilon)$, while accurately measuring a small amount of slowly varying mechanical strain due to slow fouling buildup, estimated to be approximately $50 \mu \varepsilon$ over two weeks.

Based on extensive lab testing of the fiber sensors, as well as the Inconel tube for packaging for the axial temperature measurement, it was decided that axial temperature measurement had too high risks in terms of packaging and sensor failure and potentially adversely impacting plant operation, and was thus dropped from the sensors being pursued. Extensive lab testing for FBG temperature measurement for radial temperature profile, as well as strain measurement outside the hot gas path using fiber optic sensors showed promising results. These lab-testing results are summarized in Section 4.2.1. These two sensing options were pursued for final package fabrication and installation in TECO IGCC plant in February-March 2009. The package design, installation and data collection is summarized in Section 4.2.2. 


\subsubsection{Lab Testing for Temperature and Strain Sensing using Fiber Optic Sensors}

For the two sensing options (i) radial temperature profile measurement in the RSC at levels 7 and 10 using a combination of thermocouples and fiber optic sensors, and (ii) strain measurement in top section of the RSC outside the hot gas path, extensive lab tests were performed with fiber optic sensors and packaging options to refine the options and validate the performance before fabricating the final sensor packages for installation in the plant. This section provides a brief summary of these lab-testing results.

\subsubsection{Lab Tests for Fiber Optic Temperature Sensing}

The objective of the radial temperature sensor probe was to obtain a radial temperature profile at two different levels in the RSC (levels $7 \& 10$ ) over a period of roughly 30 days of operation after startup, survive at estimated operation temperatures of $\sim 2000 \mathrm{~F}$, and withstand thermal shocks from intermittent shutdown/startup cycles over this period. To meet these objectives, a lab oven setup was put together to systematically test multiple fiber optic FBG sensors and down-select the final option to be implemented in the plant. Lab tests were performed with the FBG sensors in a temperature controlled oven at temperatures up to $1100 \mathrm{C}(\sim 2000 \mathrm{~F})$ to characterize both the short-term and longer-term performances. These tests demonstrated the short-term measurement accuracy and repeatability in the range of $10-25 \mathrm{~F}$ (comparable to type-K and type-S TCs being used as reference) and excellent survivability with temperature variations up to 2000F for more than two weeks for one of the FBG sensor prototypes. Figure 18 shows the lab setup used for short-term and long-term testing of the fiber sensor for temperature measurement.

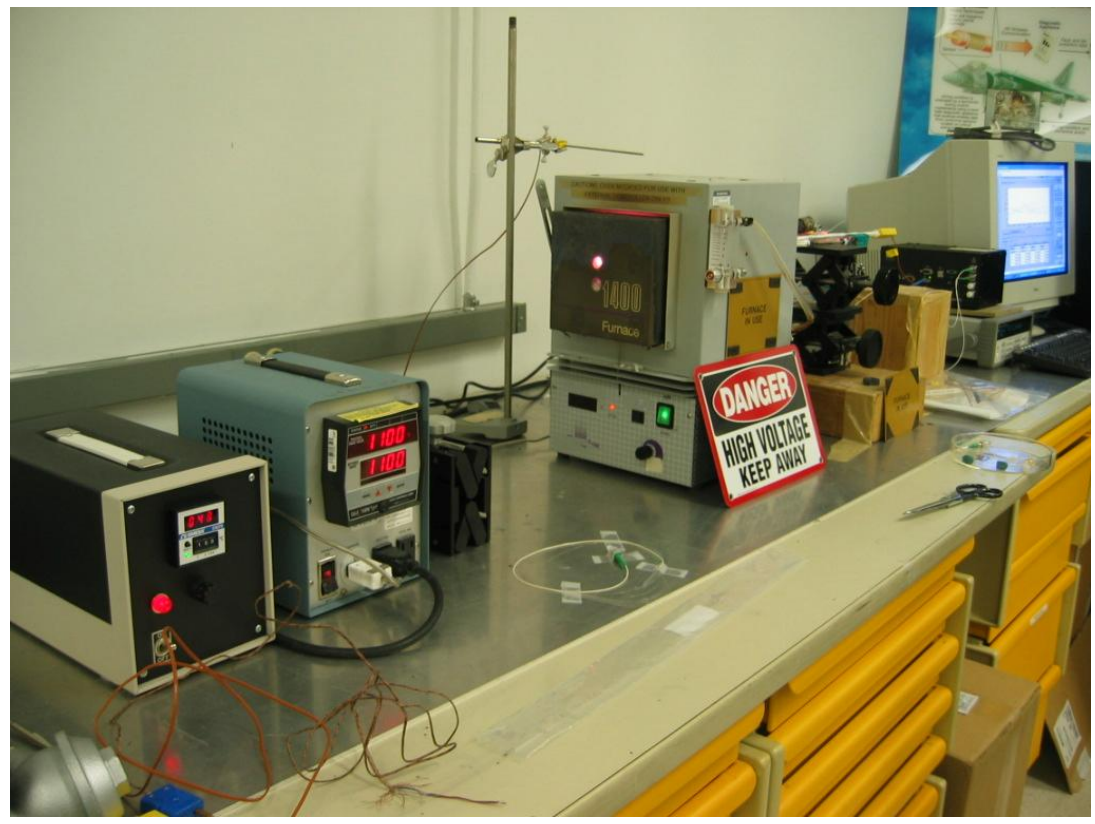

Figure 18: Lab setup for fiber optic temperature sensor short-term \& long-term testing.

Given the high risk and emphasis on survivability of the fiber sensor at these high temperatures two separate FBG sensor prototypes were studied in parallel (i) Prototype I using a fused silica, doped fiber with thermal pre-treatment/annealing, and (ii) Prototype II 
using a femto-second laser generated FBG on a non-doped quartz fiber. Initial lab tests focused on characterizing the signal behavior from both prototypes. As shown in Figure 19 , both prototypes had pretty good peak signal across the desired operating temperature range, with prototype I showing slightly better signal to noise ratio.
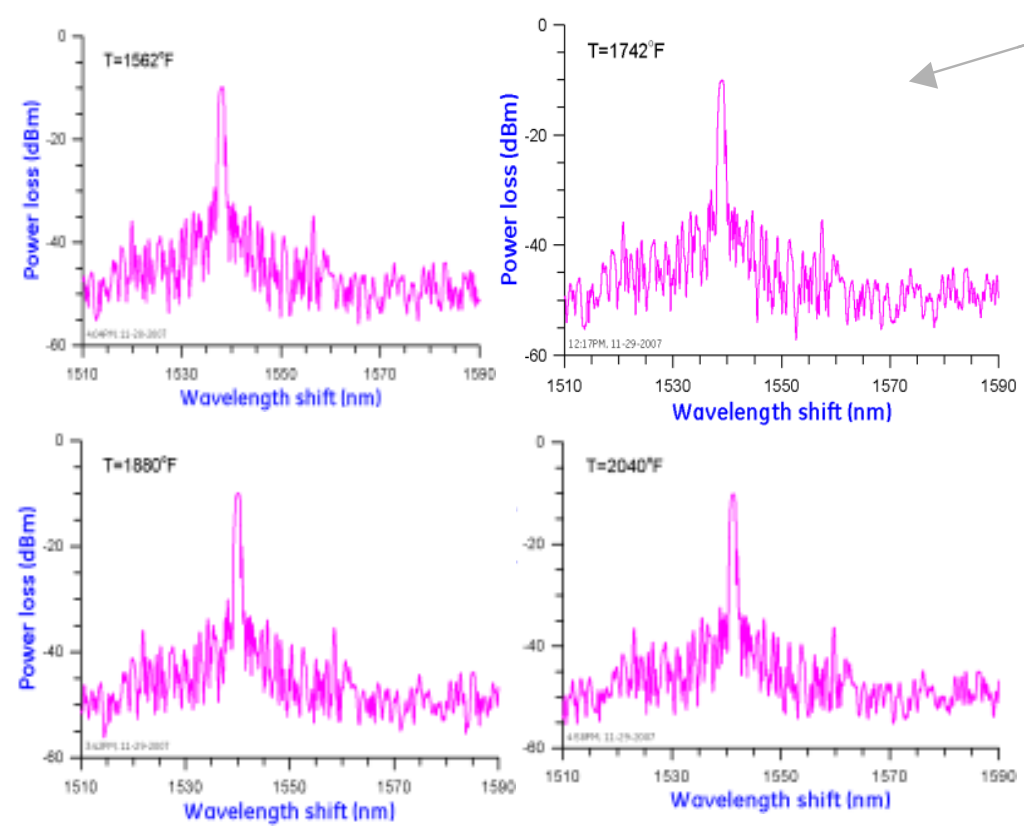

- Prototype I-Cheaper, fabricated at GRC with doped silica fiber

- Prototype II - More expensive, femtosecond laser fabrication at vendor, non-doped quartz fiber

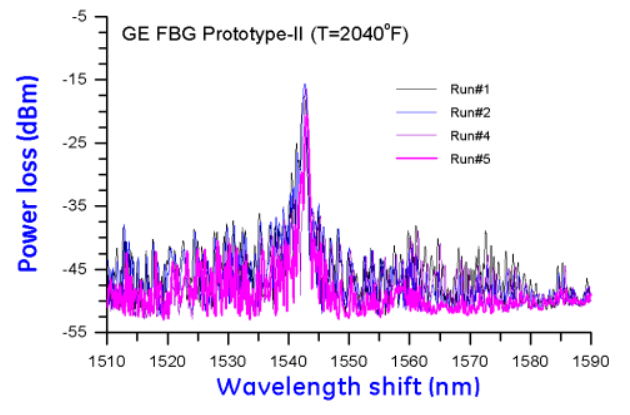

Figure 19: Signal characteristics from two prototype FBG temperature sensors
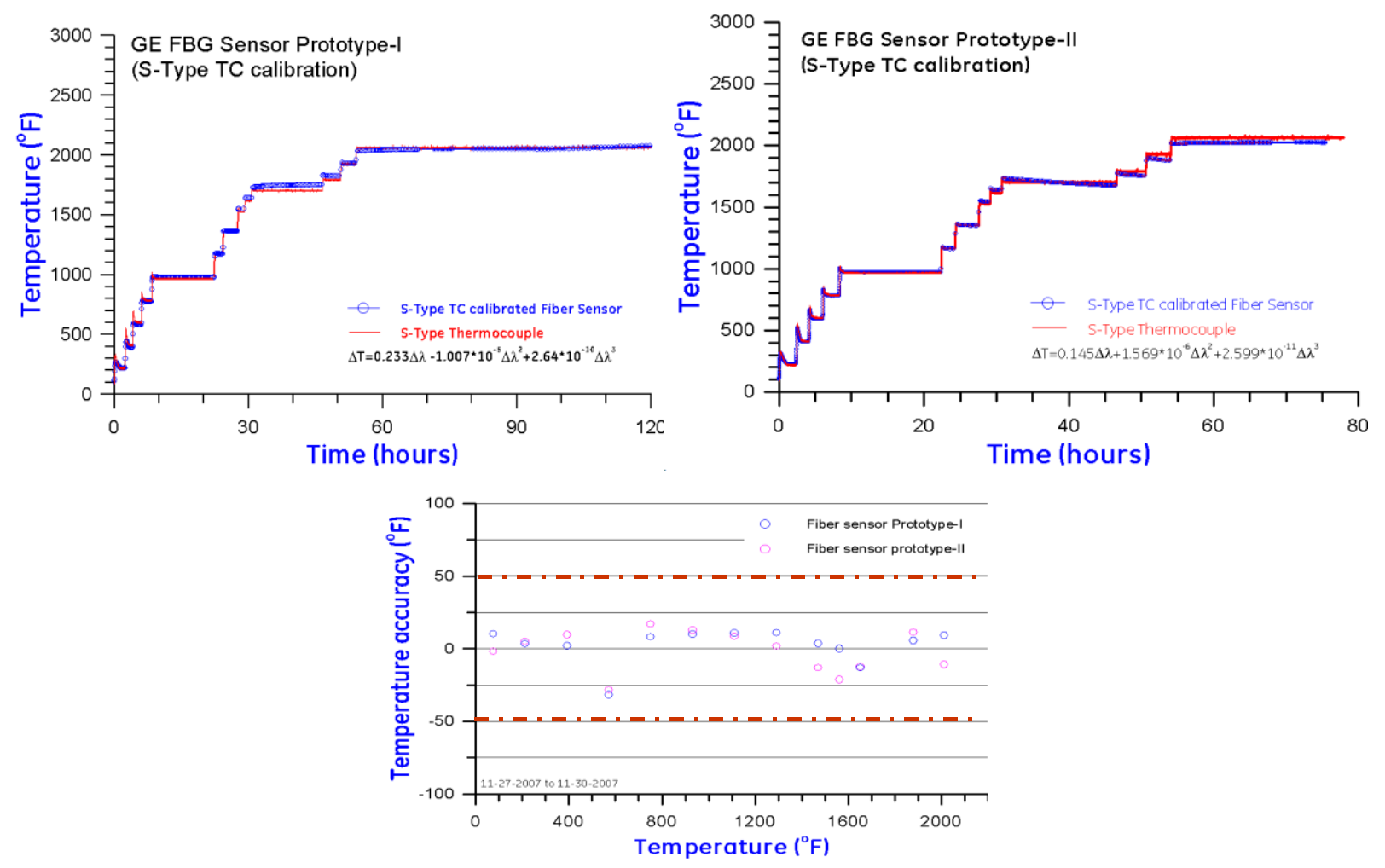

Figure 20: Short term temperature measurement errors for two FBG sensor prototypes. 
Figure 20 shows the lab test results from runs for short-term performance, where the signals from the two sensor prototypes were calibrated over the entire temperature range against a type K TC in the oven. The corresponding calibration errors are shown in the bottom plot, which shows error in the range of 20-25F. Figure 21 shows the performance of the two sensor prototypes, where the oven temperature was cycled eight times in the range 1000-2000F. A key challenge was to understand if the sensor would first survive this kind of temperature cycling, and second how repeatable the temperature measurements are across the multiple cycles. Both sensors survived the cycles well. While prototype I has smaller variation (the plot at the bottom shows 1 -sigma variation of $\sim 7 \mathrm{~F}$ ), prototype II has smaller variation at the low temperature end and higher variation at the high temperature end.
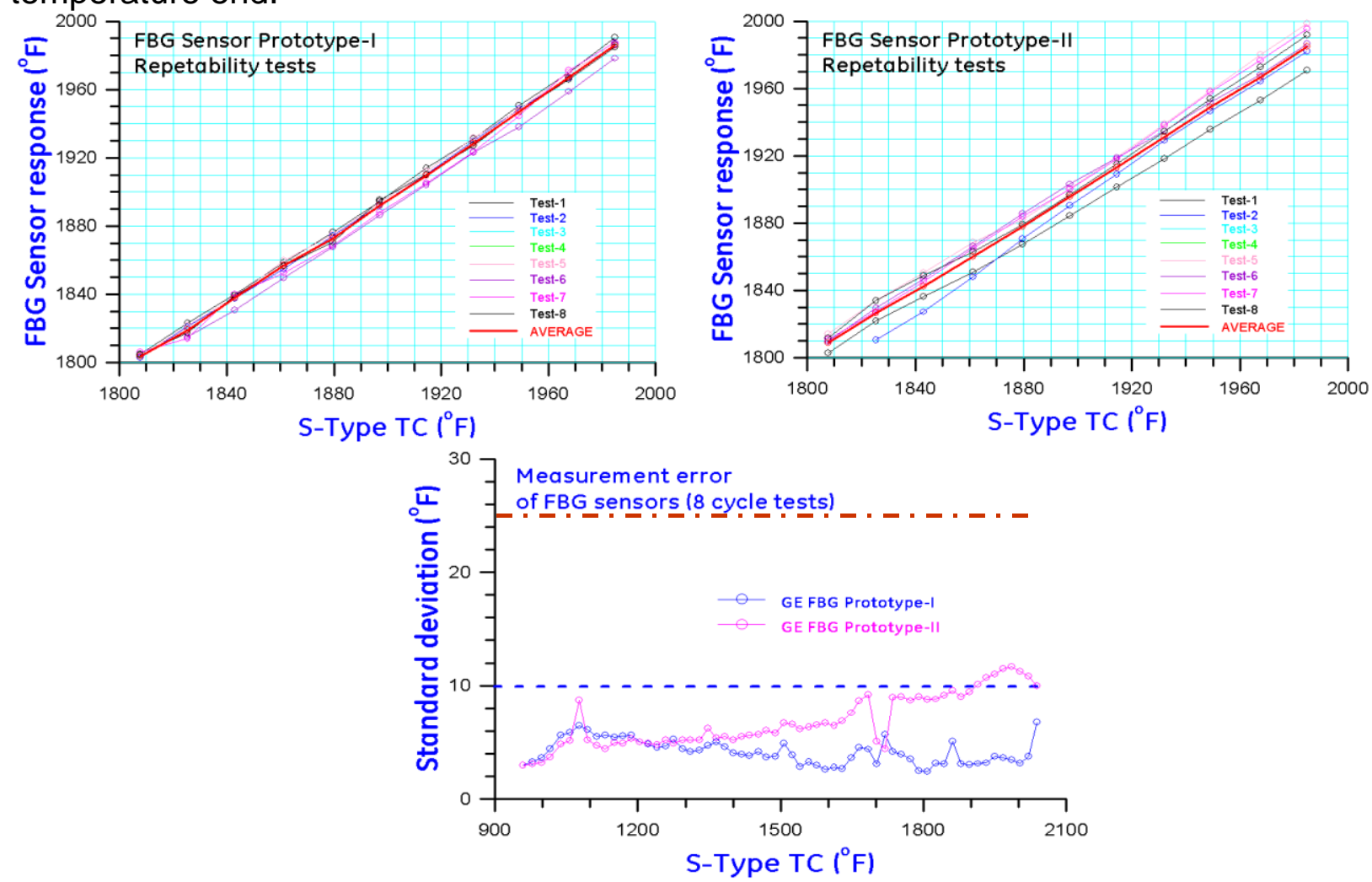

Figure 21: Short-term performance for repeatability of temperature measurement for two FBG sensor prototypes.

A critical challenge for the FBG sensor is the longer-term survivability of the sensor and stability of temperature measurement, especially at the high temperature end - for this particular application it was desired to have operation data for 30 days after startup. In particular, lab tests showed limited life of the prototype II sensor where the sensor signal was lost gradually in $2-5$ days at $2000 \mathrm{~F}$. This is likely because the entire fiber becomes crystalline and the FBG grating that relies on alternate crystalline \& amorphous layers is eventually lost. Prototype I, in contrast, showed good longer-term performance. Figure 22 shows the performance of the prototype $1 \mathrm{FBG}$ sensor when left at 2000F for 5 days continuously. The top set of plots show the first run with these sensors. In particular, the top right plot shows a zoom-in of the FBG sensor at 2000F for more than 5 days from $\mathrm{t}=120 \mathrm{hr}$ to $\mathrm{t}=250 \mathrm{hr}$. The FBG sensor shows some transient lag taking about $30 \mathrm{hrs}$ to rise from $t=120 \mathrm{hr}$ to $\mathrm{t}=150 \mathrm{hr}$ from $1980 \mathrm{~F}$ to $2000 \mathrm{~F}$. After that lag, the temperature reading 
looks quite stable. The bottom set of plots show a similar test zooming in at $2000 \mathrm{~F}$ for $24 \mathrm{hr}$ using the same sensor after repeated cycles. Again, the bottom right plot shows a stable measurement from the sensor for $24 \mathrm{hrs}$ at $2000 \mathrm{~F}$. However, this time, there is no significant lag in the sensor response. The lag in the first run indicates a possible slow phase change in the fused silica fiber during the first time at 2000F, and subsequent runs at $2000 \mathrm{~F}$ show a faster transient response since there is no additional phase change.
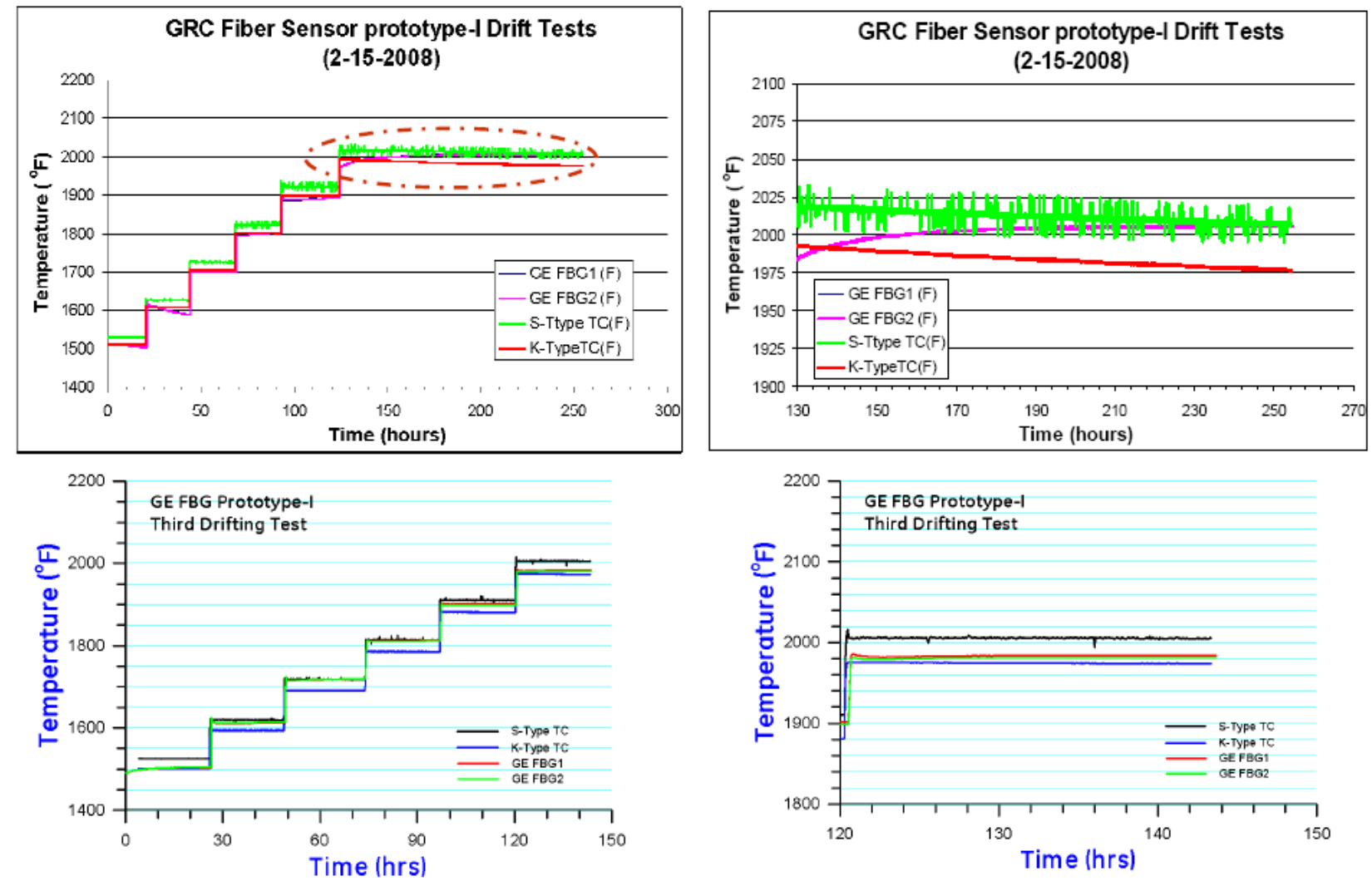

Figure 22: Longer term testing for survivability and stability of temperature measurement using FBG sensor prototype I

In summary, both prototypes I and II worked well in lab tests for short-term performances in terms of accuracy and repeatability. However, prototype II showed limited life at high temperatures (2000F) - with the sensor signal being lost over 2-5 days. On the other hand, prototype I was very promising with stable temperature measurement in lab tests for one week at a time. Moreover, repeated testing continued to show good performance over more than two weeks of accumulated testing. Based on these results, prototype I was pursued for radial temperature measurement.

\subsubsection{Lab Tests for Fiber Optic Strain Sensing}

For the strain sensing application in the RSC dome, the aim was to monitor the slow variation in the mechanical strain in the structure corresponding to gradual buildup of fouling and increasing weight of the heat transfer tubes. The key challenges were in terms of working at fairly high temperatures (nominally 600-700F but potentially up to $800 \mathrm{~F}$ ), withstanding the resulting large thermal strain (estimated to be up to $\sim 6000-7000 \mu \varepsilon$ from thermal expansion), and measuring a very small amount of mechanical strain (estimated to be less than $50 \mu \varepsilon$ ) over several weeks of operation as the fouling built up. To address 
these challenges, a fiber optic strain testing setup was used in the lab, as shown in Figure 23. The setup includes an Inconel rod heated in a temperature-controlled oven, with fiber sensors installed on the rod in the hot zone inside the oven and a commercial resistive strain gauge installed on the cold end of the rod outside the oven. The resistive sensor would not survive the desired high temperature conditions, but was used as a reference for strain measurement on the same rod. At one end the rod was fixed to a mechanical structure, while at the other end a pulley mechanism was used to implement a desired amount of mechanical strain through applied force and vary it in a desired manner.

For strain measurement with the fiber sensor, initially two options were explored in parallel, as shown in Figure 24. In particular, a big risk was the survivability of the fiber at temperatures up to 800F and corresponding large thermal strain (from thermal expansion of the rod, estimated to be about $7000 \mu \varepsilon$ ). Motivated by this, the first option shown on the left used a bi-metallic tube package designed to compensate for the thermal expansion of the rod and thus reduce the thermal strain seen by the fiber, while on other hand maintaining good contact and high sensitivity for mechanical strain. The fibers (one for strain measurement and one for temperature measurement to be used for temperature compensation) were mounted inside the tube package. Another key challenge was the bonding material to attach the fibers to the packaging for good strength and performance at these high temperatures. Three different bonding options were tested (i) high temperature solder glass, (ii) glass capillary, and (iii) high temperature ceramic adhesive. Lab tests on bonding strength and creep showed only the ceramic adhesive worked very well up to 500C without any significant creep and failure.

The second sensor option used a metalized fiber sensor directly mounted on the rod using the ceramic adhesive, as shown on the right side of Figure 24. While this direct mount option was a much simpler option, it had high risks for survivability of the fiber under high thermal strains expected.

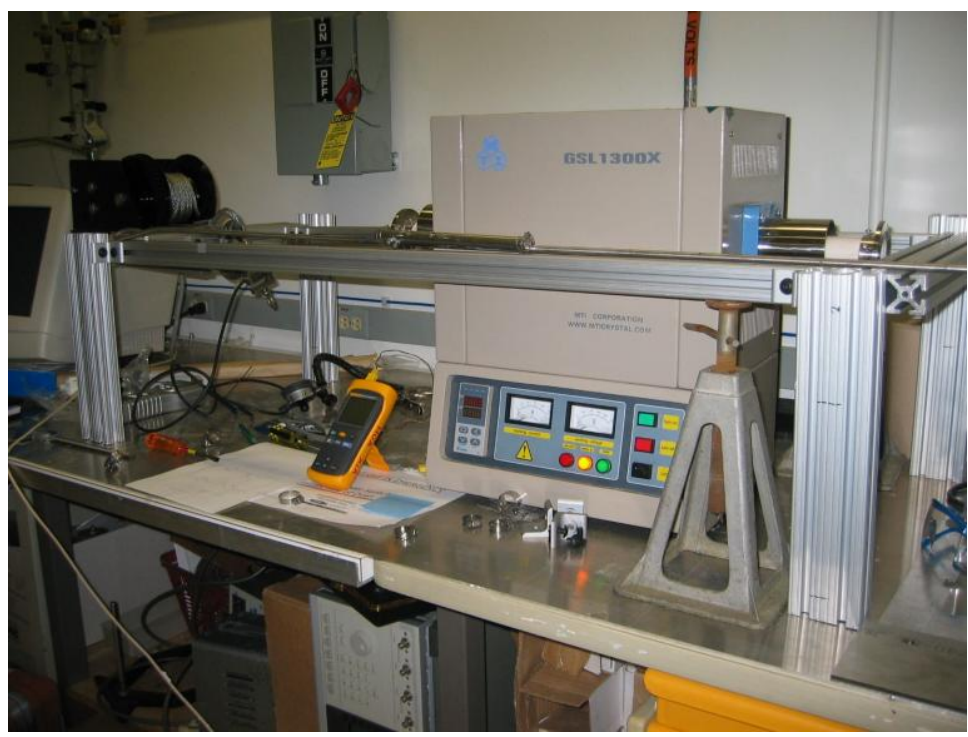

Figure 23: Lab setup for high-temperature fiber-optic strain sensor.

Initial lab tests with the bi-metallic package option showed very low signal-to-noise ratio, perhaps due to incomplete curing of the ceramic adhesive inside a confined tubular 
package. Also, repeated testing with applied mechanical strain at various operating temperatures showed inconsistent results, perhaps due to nonlinear behavior of the bimetallic package due to varying CTEs of the two metals used. Given these initial performance limitations, this option was discarded and only the direct mounted sensor option was pursued.

For the direct mounted fiber sensor, survivability of the fiber itself under the expected high thermal strain ( 6000-7000 $\mu \varepsilon)$ as the rod was heated up to 800F was a key risk.

Motivated by this, a vendor supplying a copper-coated fiber FBG sensor was identified. Another challenge was the method of ceramic adhesive based bonding of the fiber sensor to the substrate rod. In one option, it was desired to use only spot bonding using the ceramic adhesive on either side of the FBG sensor. While this option seems intuitively more reasonable to get clean strain measurements, it always led to a broken fiber during curing of the ceramic adhesive and was thus deemed risky. The other option was to encapsulate the entire FBG sensor inside the ceramic adhesive attached to the substrate rod. This method proved to be successful in lab tests for strain measurement at high temperatures.
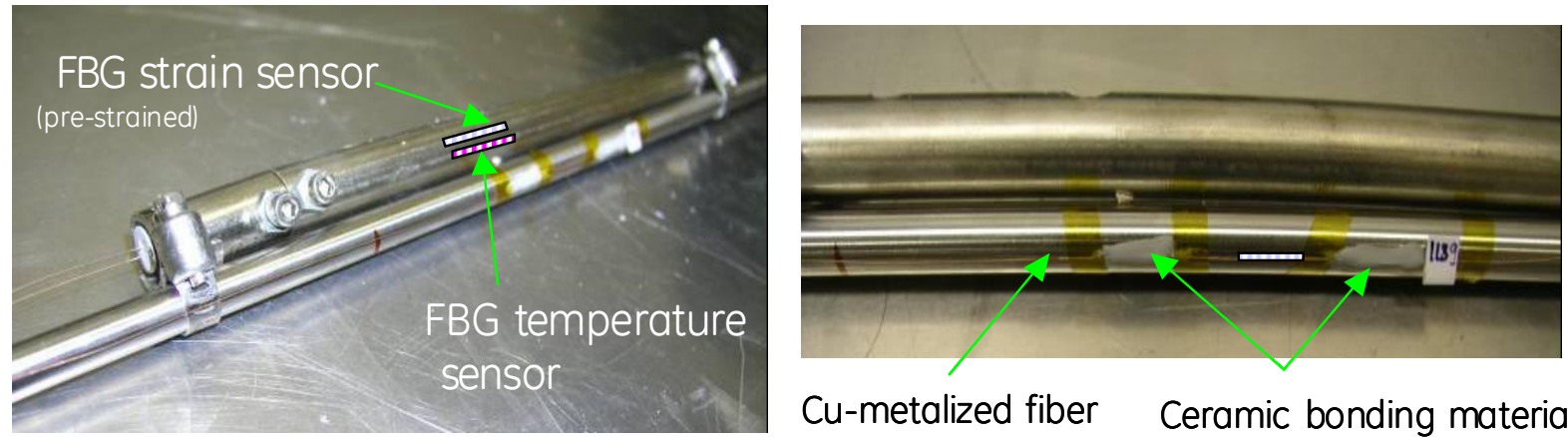

Cu-metalized fiber Ceramic bonding material

Figure 24: FBG strain sensor options (a) metalized fibers inside a bi-metallic tube packaging, and (b) metalized fiber directly mounted to substrate (rod).

Figure 25 shows strain measurement results using the metalized (copper coated) fiber FBG sensor mounted directly on the Inconel rod in the oven at different temperatures up to $700 \mathrm{~F}$, compared to the resistive strain gauge mounted on the cold end. In each case, at temperatures of 100,300, 500 and 700F, the mechanical strain was increased/decreased incrementally in steps of $15 \mu \varepsilon$, as well as large changes of $\sim 130 \mu \varepsilon$. The calibrated FBG sensor strain measurements are compared against the resistive strain gauge on the cold end, showing excellent match across these temperature conditions with a 1- $\sigma$ error of $\sim 2.2-2.8 \mu \varepsilon$, which is quite small compared to the desired mechanical strain measurement range (up to $50 \mu \varepsilon$ ) and the accuracy of the lab setup. Similar tests were performed at higher temperatures up to 1000F, to understand the limitations of this FBG strain sensing technique. The results shown in Figure 26 indicate performance deterioration, especially at 900 \& 1000F, with increased mismatch from the resistive sensor as well as increased variability in the FBG strain measurement. This is likely due to degradation in the ceramic adhesive performance due to CTE mismatch with the Inconel rod. Given that the actual temperatures in the plant were expected to be in the 600-700F range and potentially up to 800F for short durations, the copper-coated FBG sensor directly mounted onto the rod proved to be a good solution. However, the vendor supplying the copper-coated fiber went 
out of business, necessitating the search for an alternative solution. It was decided to develop a solution in-house.
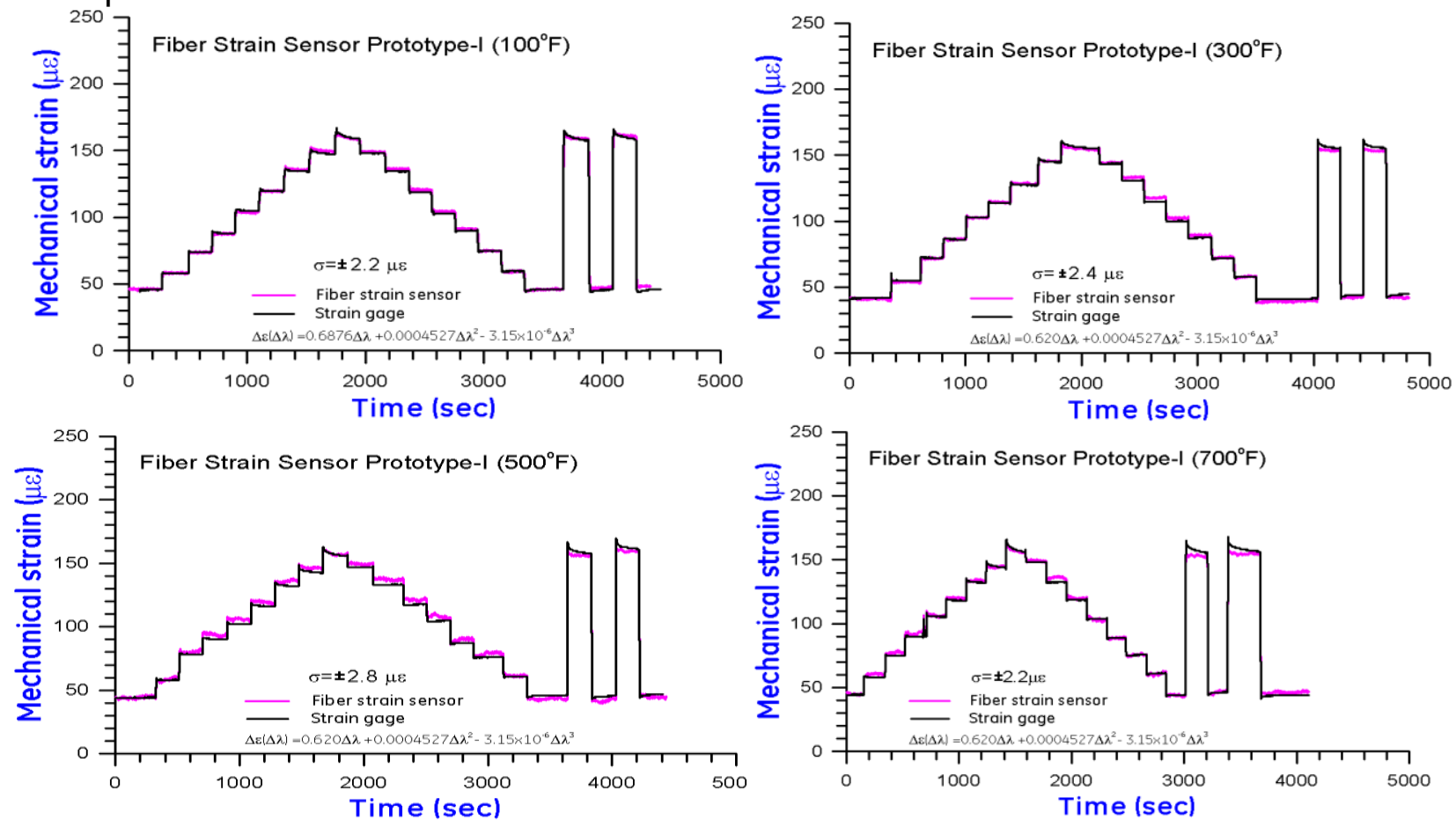

Figure 25: Performance of metalized fiber FBG strain sensor directly mounted on the rod in the hot section at temperatures up to $700 \mathrm{~F}$, compared to a resistive strain sensor mounted on the cold end of the rod.
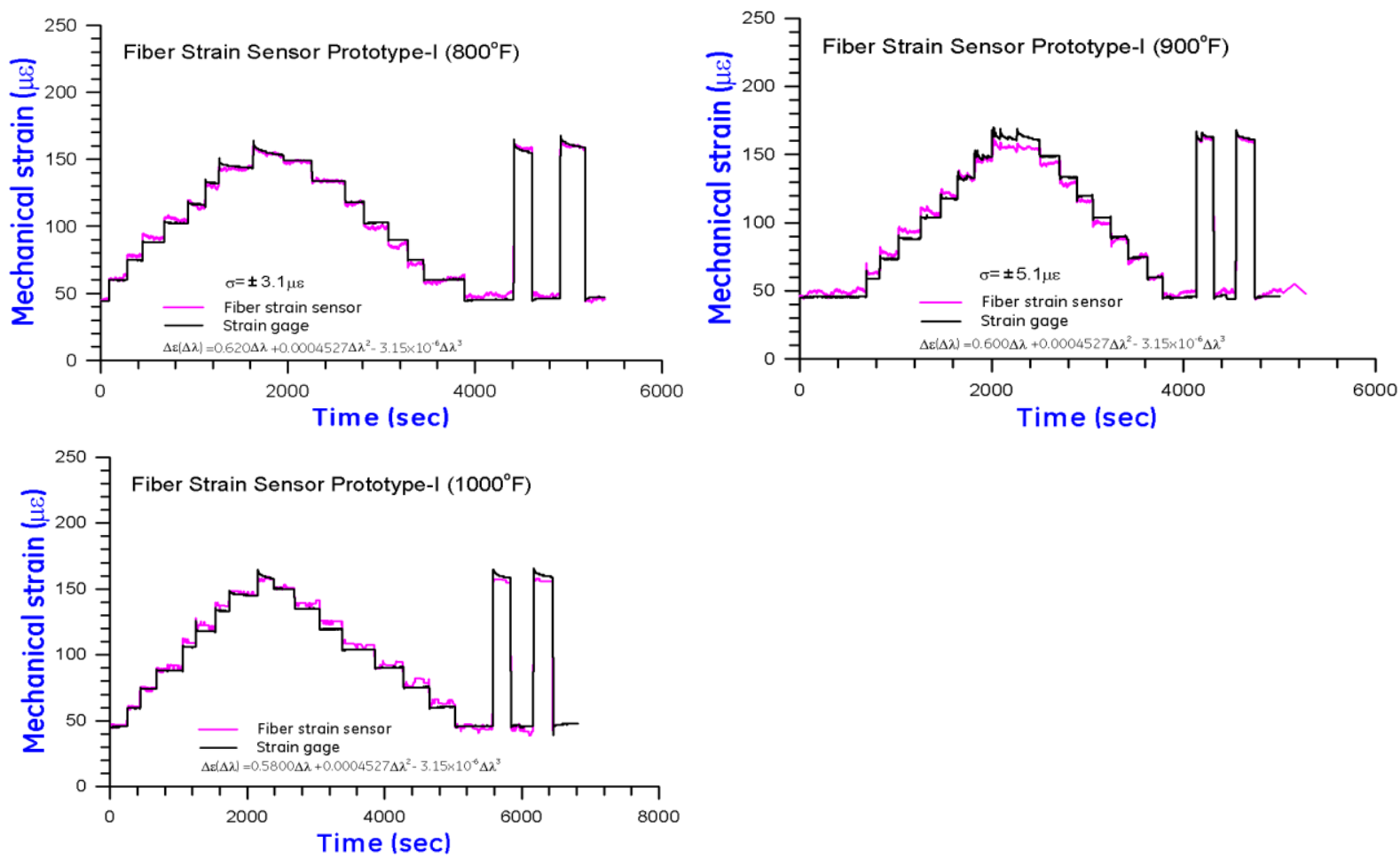

Figure 26: Performance of metalized fiber FBG strain sensor directly mounted on the rod in the hot section at temperatures between $800 \mathrm{~F} \& 1000 \mathrm{~F}$, compared to a resistive strain sensor mounted on the cold end of the rod. 
Through successive iterations, the final solution involved electroplating a thin layer of Nickel $(\sim 10 \mu \mathrm{m})$ on a regular FBG sensor, encapsulating this Ni-plated sensor within a thin stainless steel tubing, bonding both ends of the fiber to this tubing using a ceramic adhesive, and then bonding this sensor package to the rod using the ceramic adhesive.

The stainless steel tubing provided added mechanical protection as well as mitigated the interference from the curing process of the ceramic adhesive - in tests without the steel tubing, the ceramic adhesive curing led to deterioration in the FBG sensor peak signal. This final package yielded a clean FBG peak signal across the temperature range from ambient to $800 \mathrm{~F}$. This package was mounted on the same Inconel rod in the lab test setup and tested against the resistive strain gauge at temperatures up to $800 \mathrm{~F}$. Figure 27 shows the results from the lab experiments for measuring mechanical strain at $800 \mathrm{~F}$ and $600 \mathrm{~F}$. The data from 800F (shown in top plot in (a)) was used to calibrate the fiber strain sensor and the same calibration was used at $600 \mathrm{~F}$ to verify its performance against the resistive strain gauge mounted on the cold end of the rod. The comparison at $600 \mathrm{~F}$ was excellent showing consistency in fiber sensor performance at these two different temperatures.

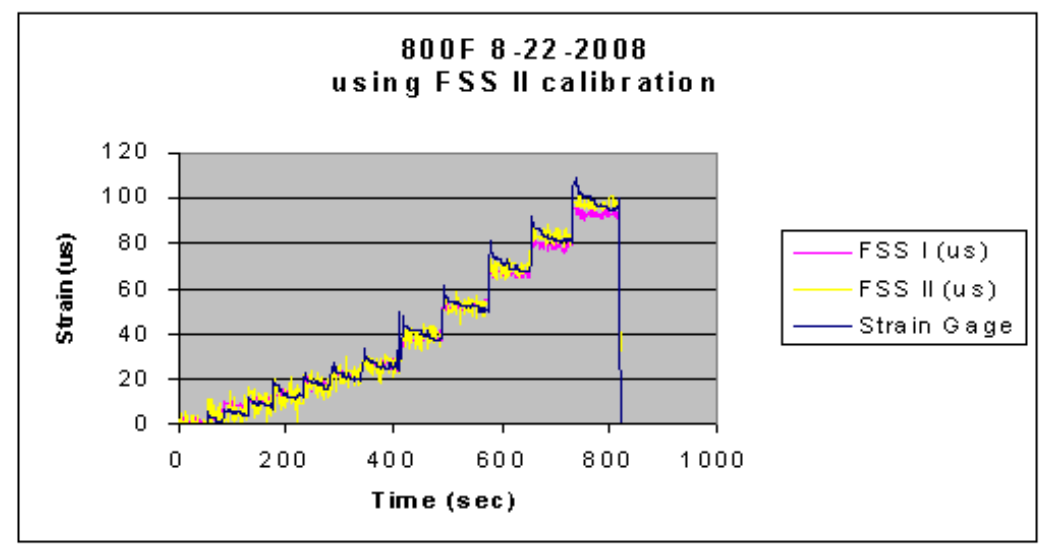

(a)

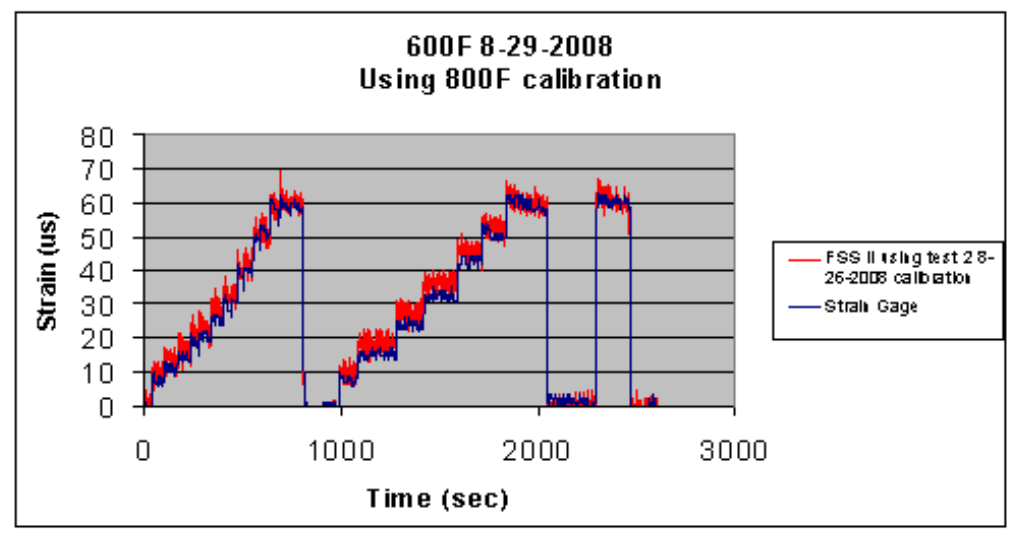

(b)

Figure 27: Lab test results with Ni-plated fiber (including FBG area) in SS tubing. Mechanical strain measurement at (a) $800 \mathrm{~F}$ and (b) $600 \mathrm{~F}$ using calibration obtained at $800 \mathrm{~F}$. 


\subsubsection{Temperature \& Strain Sensor Packaging and Installation}

For the radial temperature profile, a sensor package was designed using Hexoloy ( $\mathrm{SiC}$ ) packaging material and integrating two Type $B$ thermocouples and a fiber optic cable with ten FBG sensors distributed along the length of the probe. Figure 28 shows the overall radial package in the top with the hexoloy probe containing the two Type $B$ thermocouples and a fiber optic cable with ten FBG sensors along the length of the probe. The hexoloy probe, which was about 16" long, was integrated to the standard water-cooled soot blower lance (SBL) used in the RSC at available ports on levels 7 and 10 . There were altogether four SBL ports available, two on each level (7 and 10) - the two ports on each level are on diagonally opposite sides labeled as east and west side. The integrated TC and FBG sensor probes were installed on the east side on levels 7 and 10. In order to verify the symmetry of temperature profiles between east and west sides, another set of two hexoloy probes with a single Type $B$ thermocouple at the tip was installed on the west side on levels $7 \& 10$.
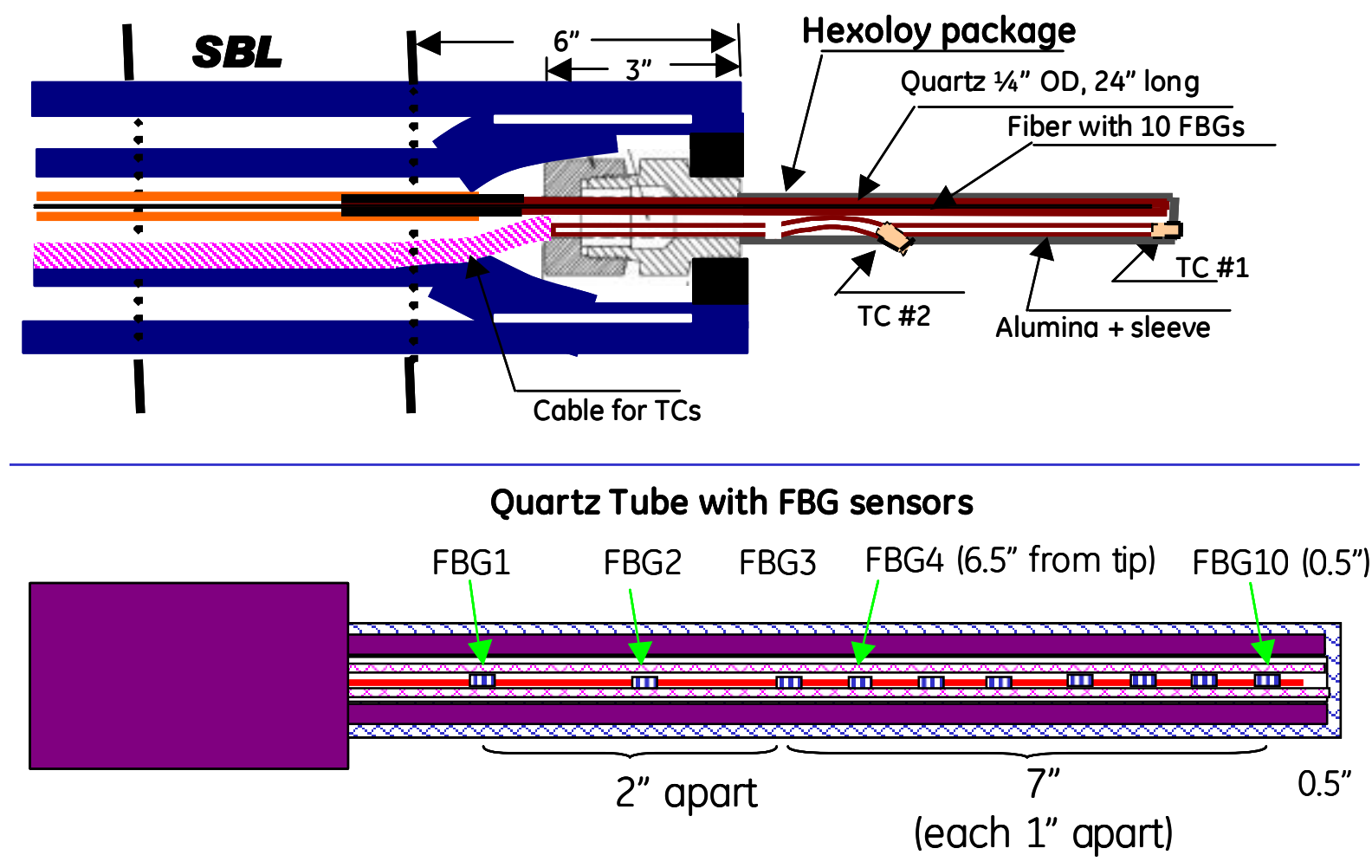

Figure 28: Radial temperature sensor hexoloy package with integrated Type B thermocouples and FBG sensors used at levels 7 and 10 in RSC. 


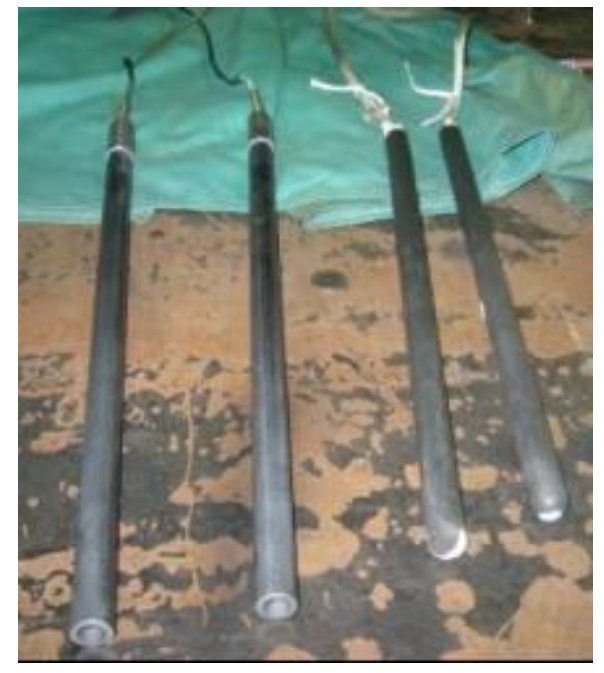

Figure 29: Four hexoloy radial temperature probes with integrated Type B TCs and FBG sensors (two probes on right) and single Type B TCs (two probes on left).

Figure 29 shows the four radial temperature probes - two probes with single Type B TCs at the tip and two probes with two Type B TCs and ten FBG sensors each. Figure 30 shows a picture of the completed sensor packages with TCs and fiber optic temperature sensors installed in a soot blower lance.

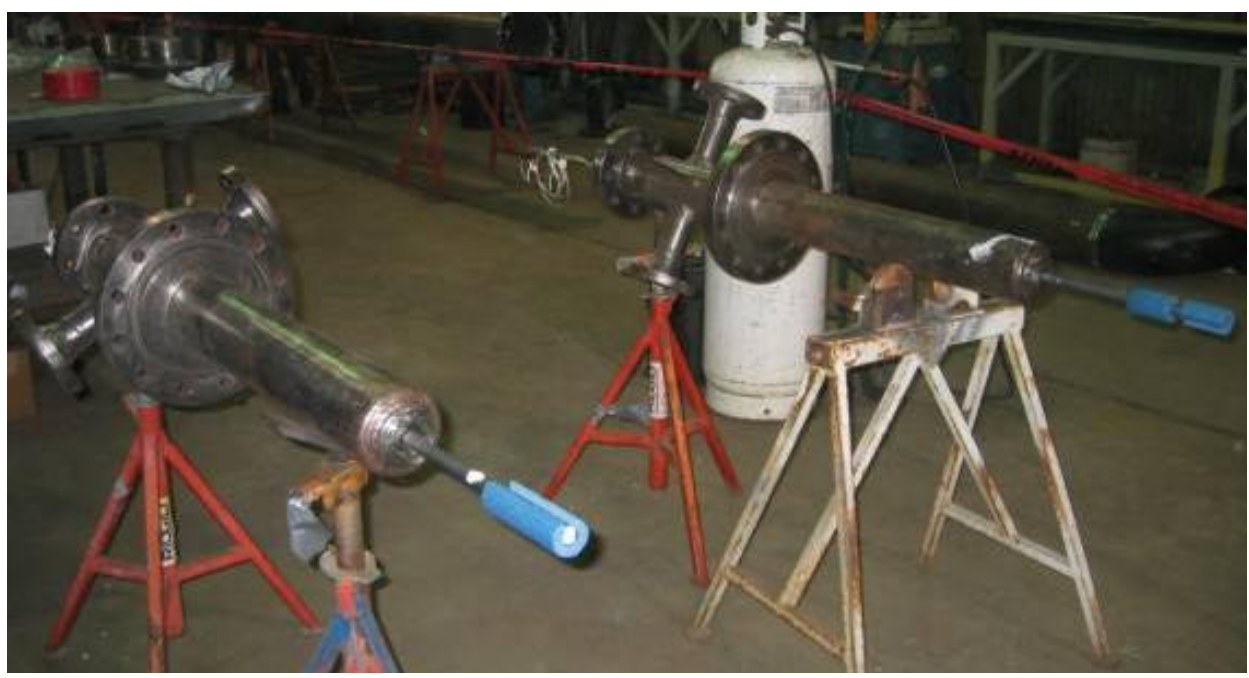

Figure 30: Soot-blower lances with temperature sensor package installed in TECO IGCC RSC.

In addition to the temperature sensing probes at levels 7 and 10 in the RSC, fiber optic strain sensors were packaged and installed on the piping support structure in the RSC dome. In particular, the final design with Ni-plated FBG sensors encapsulated in the stainless steel tubing was used and bonded to the structure with ceramic adhesive. Since the fiber FBG sensor responds to both variations in temperature as well as strain, separate Type $\mathrm{K}$ thermocouples were also installed to independently measure temperature to allow for correction of temperature variations. 


\subsubsection{RSC Data Collection}

This section presents the data collected from the temperature and strain sensors in the $\mathrm{RSC}$ - the plots included in this section show the temperature and strain variables in normalized units to protect proprietary information.
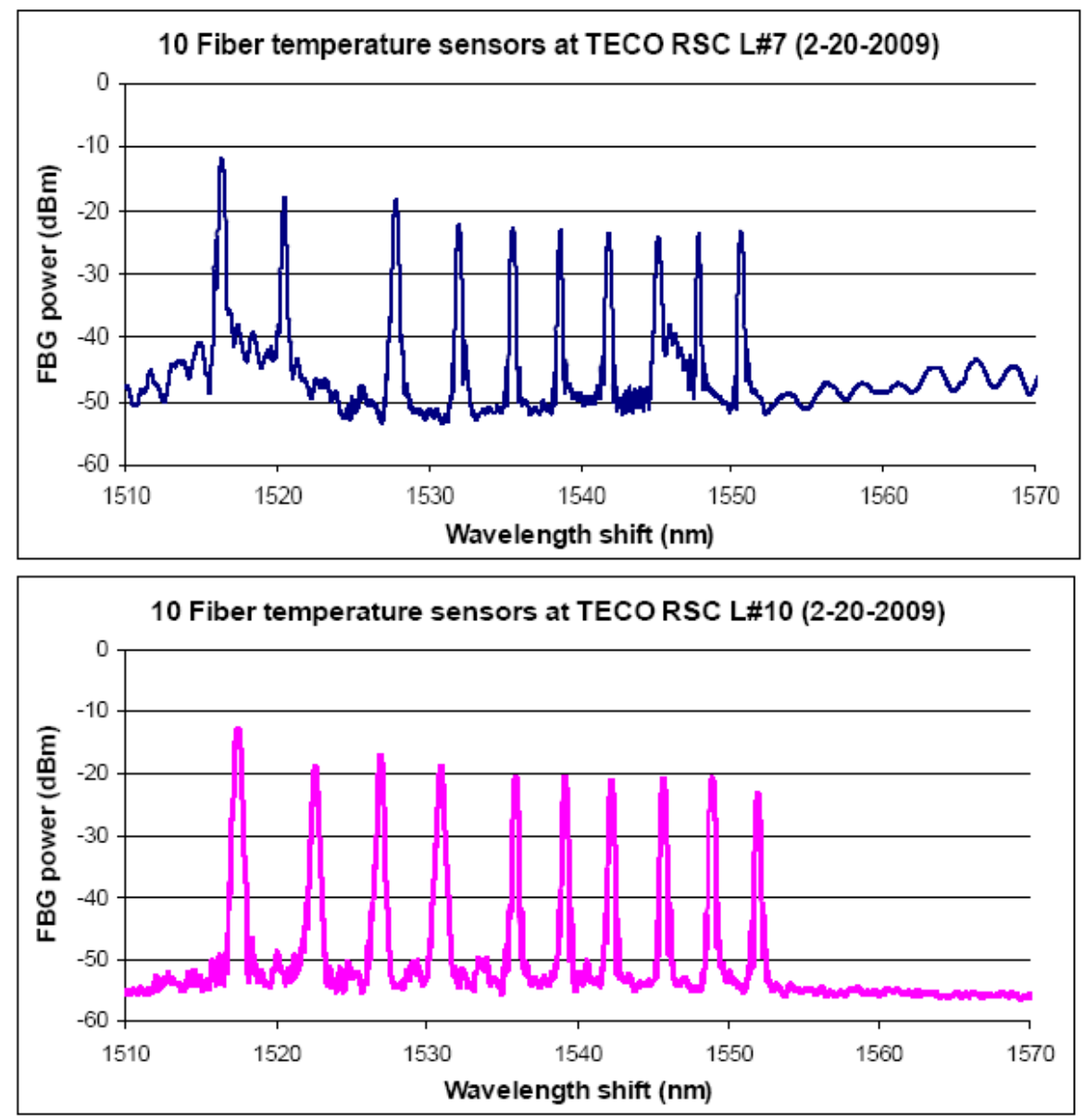

Figure 31: Spectrum data from fiber optic sensors with 10 FBG sensors for each soot blower lance installed in the TECO IGCC RSC.

Figure 31 shows the spectrum data from the 10 FBG sensors on the fiber optic cable in each sensor package for SBLs at level 7 and level 10. The two fiber optic sensor cables showed excellent signal-to-noise characteristics with a significant peak power in the range between $-15 \mathrm{~dB}$ to $-25 \mathrm{~dB}$ compared to the baseline noise of $-50 \mathrm{~dB}$, allowing for easy peak tracking as these peaks from individual FBG sensors shifted due to temperature changes. Figure 32 shows the transient profile for the 10 FBG sensors from the sensor package installed in the SBL at level 7. The bottom plot shows the calculated (normalized) temperature profile over a period of approximately five days after startup. The plots show measured temperature profile over the length of the probe as the plant operation was varied during this period. Unfortunately, on the $5^{\text {th }}$ day, the sensor probe was lost abruptly despite working very well until then - it is likely that the hot gas and slag flow from the gasifier impinged directly on the probe leading to breakage of the ceramic packaging. Temperature measurements on level 10 showed transient asymmetries between the east and west sides (see Figure 34) indicating likely acentric flow of the hot gas, increasing the likelihood of impingement on the probes at level 7 , which is approximately half way down 
the length of the RSC. As seen from the plots on the top, the peak intensities from all ten FBG sensors were very stable until the abrupt mechanical failure occurred, indicating that all the FBG sensors were performing without any degradation. The FBG sensors labeled with numbers 1 (at the probe base, i.e. the cold end) and 10 (at the probe tip, i.e. the hot end), show a significant temperature gradient along the length of the probe.

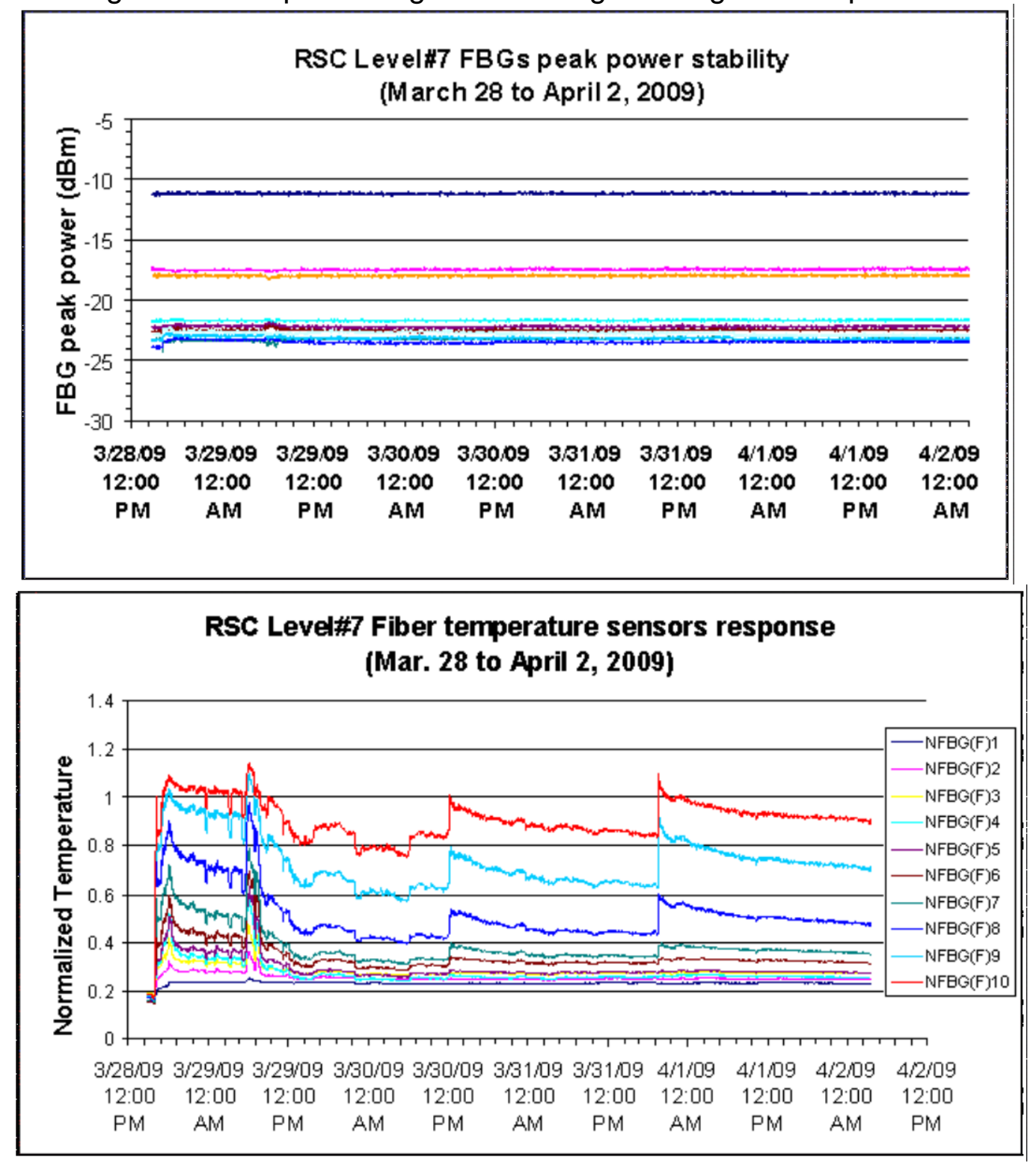

Figure 32: Peak intensity and normalized temperature from 10 FBG sensors at SBL level 7.

Figure 33 shows the peak intensity and calculated normalized temperature profiles from the FBG sensors at level 10. At this level, during ignition at startup, the transient temperatures experienced by the FBG sensors at the tip of the probe exceeded significantly well beyond the FBG design targets of $2100 \mathrm{~F}$, as tested in lab ovens, leading to failure of the FBG sensors 9 and 10, within 1.5 inches of the probe tip. FBG sensors 1 8 , where sensor 8 is 2.5 inches from the probe tip performed well and continued to measure temperature data after multiple weeks of operation. The top plot shows the peak 
intensity of the FBG sensors 1-8. Note that for about two days between April 5 and April 7, the data was lost due to a power failure in the desktop computers recording the data.
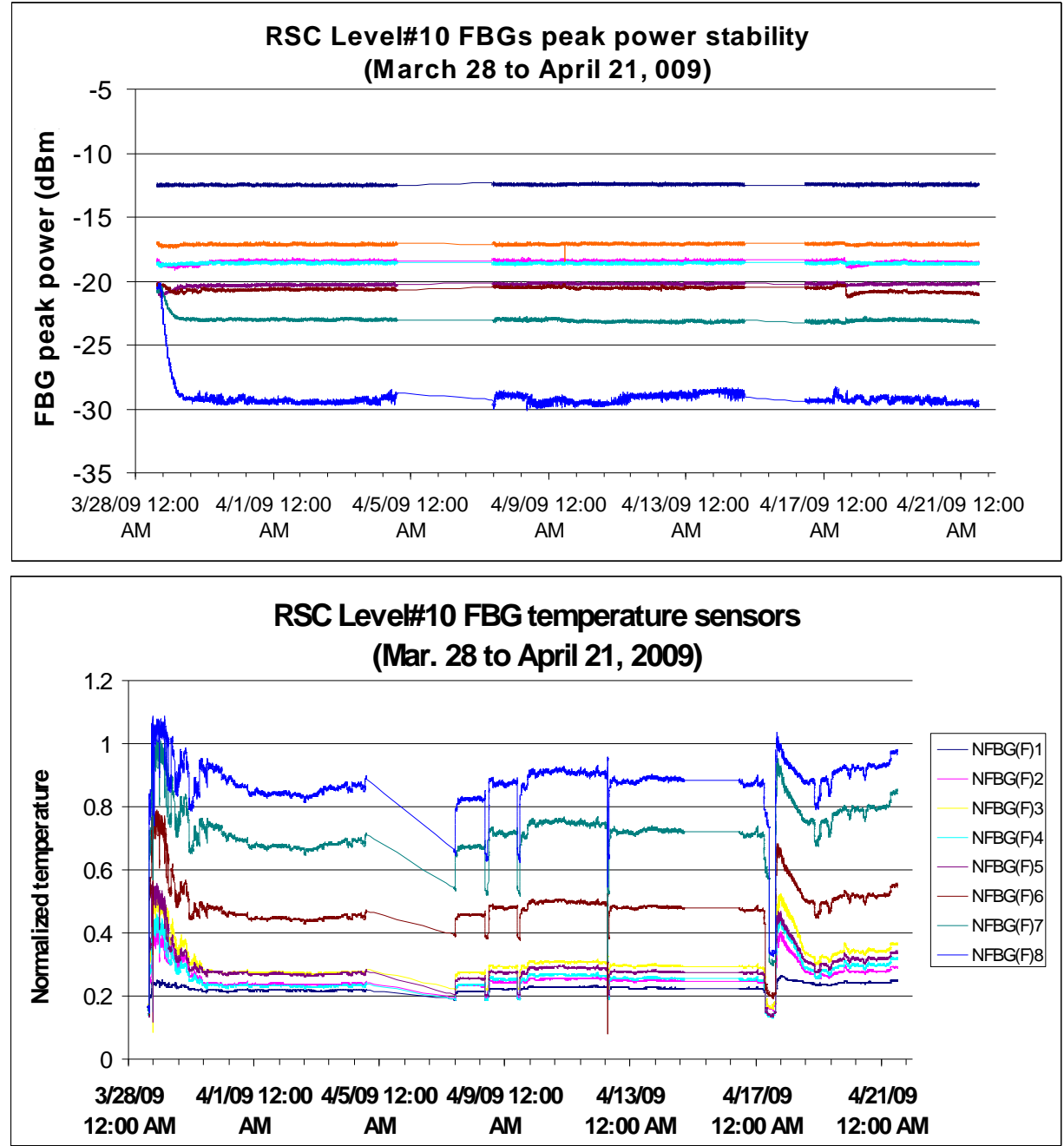

Figure 33: Peak intensity and normalized temperatures from FBG Sensors at level 10.

Figure 34 shows the plot for measured (normalized) temperature by the TCs as well as the FBGs in the SBLs at level 10. Note that the temperatures measured by the TCs at the tips on both the east (probe with TC and FBG sensors) and west (probe with single TC at tip) sides are quite close, especially towards the later period when the plant operation stabilized. However, there were periods in the beginning of operation where the two sides yielded temperature changes in opposite directions, e.g. around March 30, the TC on west side showed a temperature increase while the TC (and FBG sensors) on east side showed a temperature decrease, indicating a possible asymmetric flow of the hot syngas in the RSC. Note also that the temperature profiles measured from the FBG sensors are consistent with the TC temperatures on the east-side probe, except for right after startup when the FBG sensors experienced higher temperatures than the TC at the probe tip. 

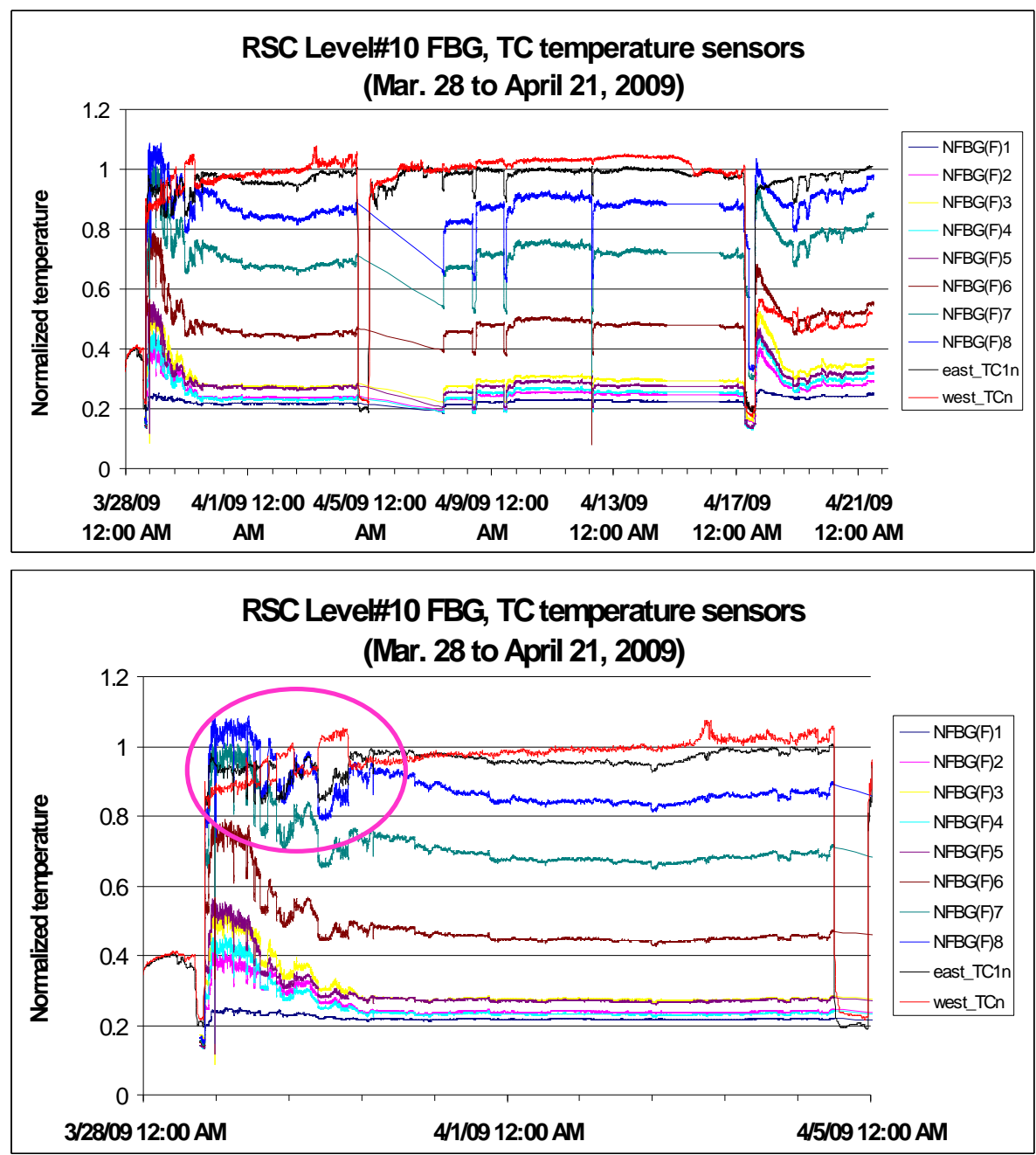

Figure 34: Normalized temperature from TCs and FBG sensors at level 10.

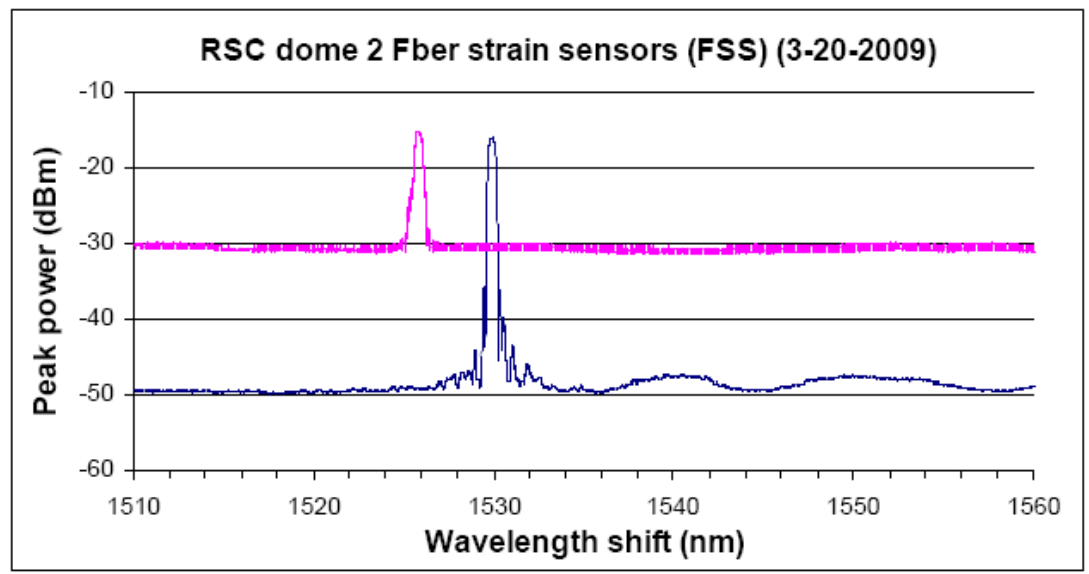

Figure 35: Spectrum data from two fiber optic FBG sensors used for strain sensing.

In addition to the temperature sensor probes installed in the RSC, fiber optic strain sensors were also installed outside the hot gas path to monitor slow evolution of small mechanical strain on key components due to gradual fouling buildup. Figure 35 shows the nominal frequency spectrum data from two fiber optic FBG sensors mounted at the same 
location. Since the peak wavelength shift from the FBG sensors corresponds to both temperature variation as well as strain variation, type $\mathrm{K}$ thermocouples were also installed to monitor the temperature in order to deduct the effect of temperature variation to calculate mechanical strain. During the initial sharp temperature transients at startup, the measured wavelength shift by the FBG is assumed to be due to temperature effects alone (temperature + thermal strain) and is used to calibrate for temperature effect on the measured strain from the FBG sensor. Thereafter, as the temperature stabilized over the subsequent period, this calibration was used to compensate for small temperature fluctuation and obtain the mechanical strain. Figure 36 shows the normalized temperature measured by the Type $\mathrm{K}$ thermocouple for approximately six weeks of operation after startup. There is a sharp rise in the temperature during initial startup, and thereafter the temperature is fairly stable, except for three intermittent changes during temporary shutdowns. Figure 37 shows the calculated mechanical strain evolution during the same six-week period after March 29, when the temperature was relatively stable. There was a short plant shutdown and loss of data collection in the middle of this period. The calculated mechanical strain from the FBG measurement shows a gradual increase in mechanical strain over the six-week operation to about $200 \mu \varepsilon$. This is consistent with the previously estimated $50 \mu \varepsilon$ from fouling over two weeks. Note that during the intermittent shutdowns, the calculated mechanical strain is incorrect due to the sharp excursions in the temperature, but the mechanical strain profile is fairly continuous across those shutdowns. Also, the FBG sensor data collection was lost for two days around April 4, due to loss of power to the data acquisition computers. Overall, the measured mechanical strain provides a direct estimate of the fouling buildup on the heat transfer area, and is consistent with previously estimated fouling and mechanical strain buildup.

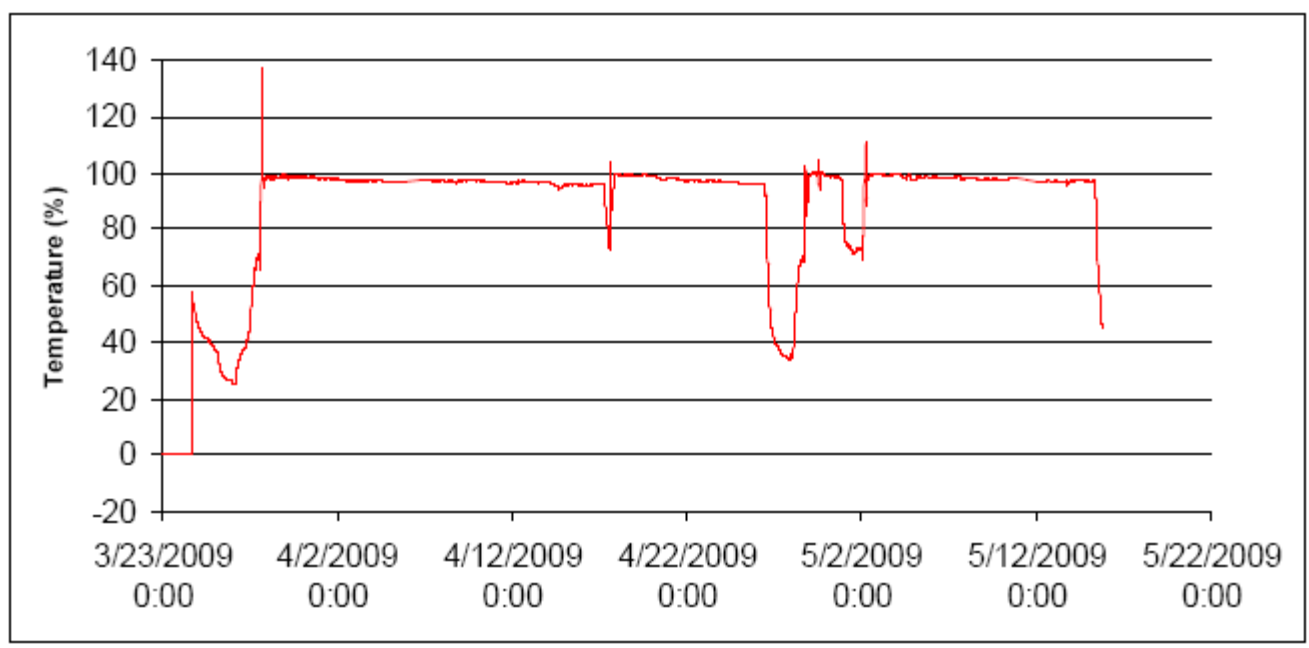

Figure 36: Measured (normalized) temperature in the RSC dome. 


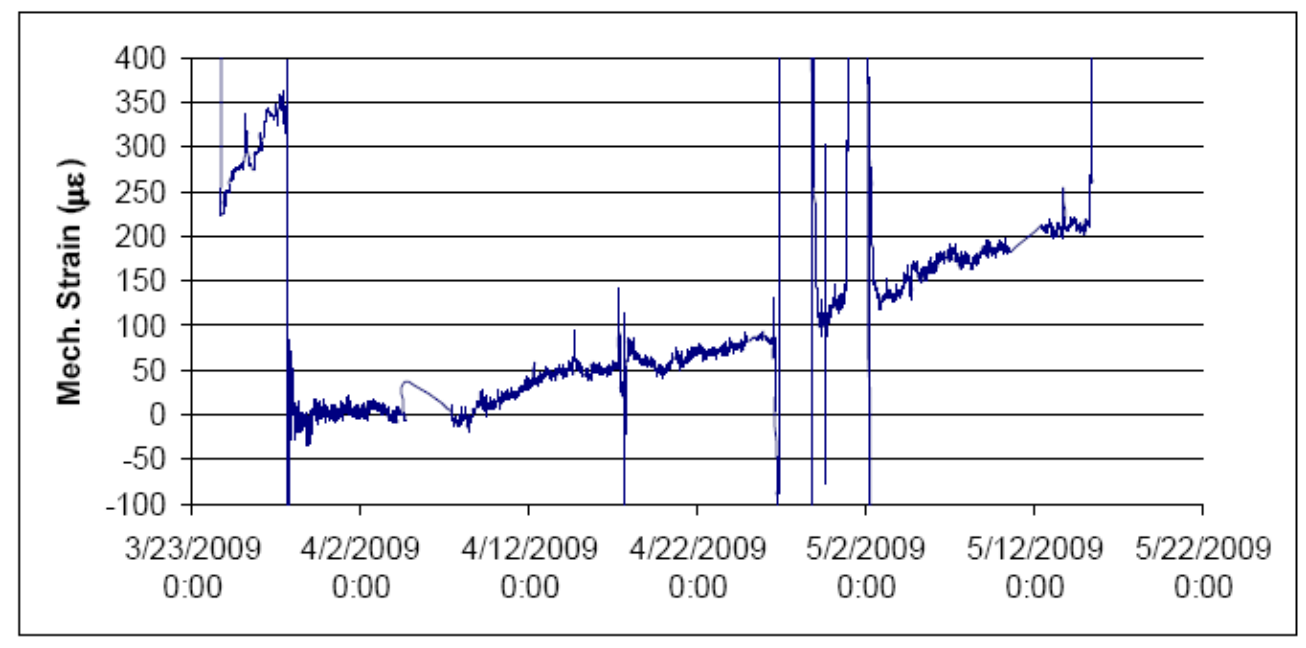

Figure 37: Calculated mechanical strain from FBG sensor, during stable thermal operation.

\subsection{Model Validation with TECO IGCC Plant Data for Nominal Operation}

The RSC model had been validated using available startup preheating data from TECO IGCC plant (see Figure 15) - the thermal profiles calculated by the RSC preheating model match very well with the tube and shell temperature measurements in the plant. For nominal plant operation at baseload, additional plant operation data, e.g. gasifier operation conditions to enable simulating and comparing the RSC model output with measured data, were obtained in 2009 Q3. In 2009 Q4, the developed gasification section model was modified for simulation with the TECO IGCC plant configuration and operation conditions to validate the model against available measurements on temperatures in the RSC as well as syngas composition.

More specifically, in October 2009, TECO plant operation data and temperature measured by the installed thermocouples and FBG temperature sensors for tests in March and April 2009 were obtained through GE Energy Gasification team. The plant operation data included mainly the flow rates, temperatures, pressures, water levels and syngas composition in the gasification section, i.e., gasifier, RSC, convective-syngas cooler (CSC) and scrubber (a sampling data every 15 minutes). The recorded data for the installed thermocouples at Level 10 is longer than those at Level 7, as mentioned in the earlier section.

Table 1: Data from TECO Plant Tests in March and April 2009

\begin{tabular}{|l|l|l|l|l|}
\hline Data type & Location & Start Time & End time & Data Frequency \\
\hline Operation & Gasification Section & $8: 00,3 / 20$ & $8: 00,4 / 30$ & Every 15 minutes \\
\hline & & & & \\
Installed TC & Level 10 and level 7 & $16: 01,3 / 26$ & $1: 53,4 / 10$ & Every 10 seconds \\
\hline & & & & \\
Installed FBG & Level 10 and level 7 & $14: 49,3 / 28$ & $14: 27,4 / 9$ & Every second \\
\hline
\end{tabular}

Based on data availability, process transition and the objective of steady state validation, two time periods are picked on preliminary data analysis: April 1, 12:00 24:00, which has 
both Level 10 and Level 7 measurement data, and April 9, 2:00 8:00, where only Level 10 measurement was available. Due to the limitation in operation data, the feed water and steam flow information for CSC is not available, only the total steam flow from the HP drum, i.e. the combined steam generation from RSC and CSC is available. The CSC is unique to the TECO plant configuration and is not included in the general gasification plant configuration modeled, and hence is excluded from the model comparison. The two identified steady-state operation periods mentioned above are similar in general. However, the syngas composition information for April 1 time period were out of range, and thus, this data was not picked for model validation purpose. Process condition on April 9 was chosen for model validation purpose. For this period, the measured gas turbine power output indicates operation at full baseload condition.

The gasification section model was modified to run the TECO configuration. More specifically, for the gasifier inputs, the coal flow, water flow in slurry, oxygen flow and feed temperature were specified based on measurements, and the $\mathrm{CO}_{2}$ flow was eliminated (no $\mathrm{CO}_{2}$ recycle in TECO plant). Modifications on RSC model included specifying the RSC diameter and length for TECO plant design. Main changes for RSC HP drum and the scrubber models were their operation pressures, where the measured pressures were used as the setpoints for PID controllers in the model. These PID controllers ensured that the scrubber and HP drum pressures in the model matched the measured data from the plant. Initially, the model operating conditions were ramped from high throughput and pressure conditions used for a general larger scale reference plant to the baseload conditions for TECO IGCC plant to obtain initial conditions for the model consistent with TECO IGCC plant conditions. The preliminary results from the model showed a good match for the gasifier temperature, about $70^{\circ} \mathrm{C}$ lower RSC syngas exit temperature compared to measurement, and the scrubber syngas compositions matching reasonably well to the measured ones (dry basis), $\mathrm{CO}$ about $4.5 \%$ lower, $\mathrm{H}_{2}$ about $1.8 \%$ higher, $\mathrm{CO}_{2}$ about 3\% higher and $\mathrm{CH}_{4}$ about 90 ppm lower.

These preliminary results indicated that the gasifier model matched well with the operation data - this is not surprising since the original gasifier model from which the reduced-order model was derived had been validated against previous TECO IGCC plant operation data. On the other hand, the lower RSC exit temperature predicted by the model indicated a higher heat transfer from the syngas to water/steam in the RSC model compared to the plant. Finally, the extent of the WGS (water gas shift) reaction modeled in the RSC was too high, leading to lower $\mathrm{CO}$ and higher $\mathrm{CO}_{2}$, while the methanation reaction rate (CO and $\mathrm{H}_{2}$ converts to $\mathrm{CH}_{4}$ and water) was not enough. It should be mentioned that the TECO plant configuration is unique and a little different from a general reference plant gasification section. In particular, the TECO plant has no quench after the RSC; rather a CSC is used to extract additional heat from syngas as HP steam. Thus, the temperature and flow measurements downstream of the RSC are not directly comparable with the model (the flow measurement includes moisture which depends on the temperature, pressure and corresponding saturation conditions). On the other hand, the syngas composition on dry basis for key components like $\mathrm{CO}, \mathrm{CO}_{2}, \mathrm{H}_{2}$ and $\mathrm{CH}_{4}$ are unchanged between the RSC and the scrubber exit, and these can be compared with the model.

To match the RSC exit temperature, the fouling factor in the model was increased iteratively until the increased fouling led to sufficiently reduced heat extraction in the RSC 
and thereby a matched RSC exit temperature. Once the RSC exit temperature was matched, i.e. the RSC temperature variation from inlet to outlet was matched, the scale factors for the WGS and methanation reactions were adjusted to enable matching the syngas composition measurement for $\mathrm{CO}, \mathrm{CO}_{2}, \mathrm{H}_{2}$ and $\mathrm{CH}_{4}$ (on dry basis) very closely. Table 2 shows a summary of the validation results, showing the percent error between the model prediction and measurements at the baseload condition. From these validation results, it is clear that the gasifier model matches pretty well with the TECO IGCC plant operation data, and after a few parameter adjustments, the RSC and scrubber models also match close to the TECO plant operation data.

\section{Table 2: Validation Results}

\begin{tabular}{|c|c|c|}
\hline & \begin{tabular}{|c|} 
Difference \\
between model \\
and \\
measurement
\end{tabular} & Comments \\
\hline \multicolumn{3}{|l|}{ Gasifier } \\
\hline $\mathrm{T}(\mathrm{C})$ & $-1.09 \%$ & \\
\hline $\mathrm{P}$ (atm, abs) & $5.79 \%$ & \\
\hline \multicolumn{3}{|l|}{ RSC } \\
\hline $\begin{array}{l}\text { T at level } 10 \\
\text { (C) }\end{array}$ & & $\begin{array}{l}\text { point mesurement vs. } \\
\text { average from 1-D } \\
\text { model }\end{array}$ \\
\hline T at exit ( $\mathrm{C}$ ) & $-0.51 \%$ & \\
\hline $\begin{array}{l}P \text { at exit (atm, } \\
\text { abs) }\end{array}$ & $1.65 \%$ & \\
\hline \multicolumn{3}{|l|}{ Scrubber } \\
\hline $\begin{array}{l}\text { Syngas flow } \\
\text { (mol/sec, dry) }\end{array}$ & $-7.05 \%$ & \\
\hline \multicolumn{3}{|l|}{$\begin{array}{l}\text { Syngas } \\
\text { composition } \\
\text { (dry) }\end{array}$} \\
\hline $\mathrm{CO}$ & $-0.61 \%$ & \\
\hline $\mathrm{CO} 2$ & $4.23 \%$ & \\
\hline $\mathrm{H} 2$ & $-0.72 \%$ & \\
\hline $\mathrm{CH} 4$ & $-5.05 \%$ & \\
\hline
\end{tabular}

In addition to the RSC exit temperature, temperature in the RSC at level 10 was also available from the installed thermocouple/FBG sensors. It should be pointed out that the temperature data from level 10 couldn't be directly compared with the model calculation result since the model is a 1-D (axial variation) model that does not include any variations in the radial direction. The 1-D model only provides an average (i.e. a mass weighted cross-sectional average) temperature along the length of the RSC. A 3-D model that describes flow and temperature variation in the RSC could be used to directly compare with the measured temperature at level 10. However, this is outside the scope of this program. 

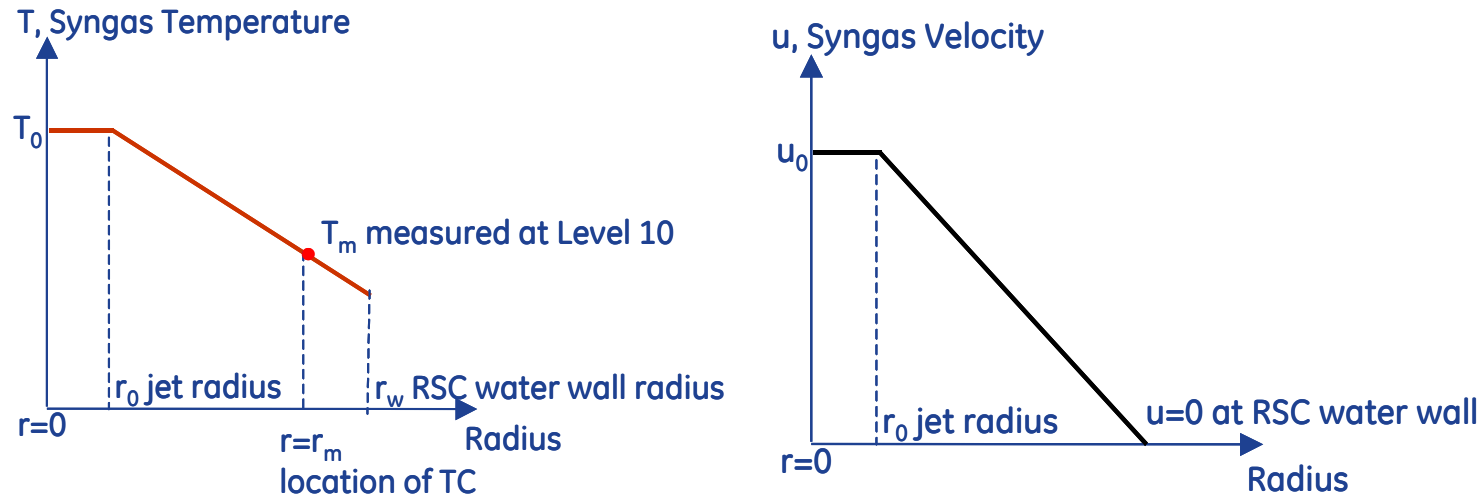

Figure 38: Radial distribution for temperature (left) and syngas axial velocity (right) assumed at Level 10.

To validate the 1-D model, the measured temperature at level 10 was used to approximate the average temperature at level 10 and compared with model prediction. In particular, a simplified linear radial profile for temperature and syngas flow velocity, as shown in Figure 38 , was assumed. In particular, for the linear temperature profile, the core temperature $T_{\text {o }}$ is the same as the gasifier exit temperature while the TC/FBG measurement provides $T_{m}$ at the corresponding radial location $\mathrm{r}_{\mathrm{m}}$. The assumed linear flow and temperature profiles are used to estimate the corresponding mass-weighted average temperature at level 10. The mismatch between the calculated average temperature and the 1-D model predicted value was about $6 \%$, which is acceptable given the measurement accuracies and simplified estimate of average temperature from point measurements. In reality, the velocity and temperature profiles are expected to be a bit different from the assumed linear profiles.

\section{Task 2 - Sensing System Design}

In Task 2, the key objective was to design a comprehensive sensing system for the gasification section to provide online, real-time information about key process variables for controls relating to performance objectives as well has key operational constraints, e.g. syngas composition, carbon conversion, slag viscosity, etc. It is very challenging and often not practically feasible to measure all these variables directly for monitoring \& control purposes through online sensors, especially due to the very harsh environment in the gasification section. Motivated by this, in this task, a systematic model-based analysis and design approach was developed for a comprehensive sensing system as shown in Figure 39. More specifically, an optimal combination of critical online sensors is complemented by online model-based estimation (or virtual sensing) to provide direct or inferred measurements of all critical variables. A key initial step in this direction was to use systematic model-based analysis, using the model from Task 1, to identify the optimal combination of online sensors in this architecture.

In phase II of the program, initially the nonlinear transient model of the gasification section was used as the basis to analyze the sensing system design and performance. More specifically, the nonlinear model was linearized at baseload nominal operating condition. The linear model was then used in a Kalman filter analysis to study the performance of the sensing system in terms of expected accuracy of estimation of key unmeasured process 
variables, as function of key sources of errors/uncertainties in the model as well as the online sensors. Kalman filter is an established model-based estimation technology that provides the best estimate of unknown "state" variables in a dynamic system using online measurements of system outputs, while systematically accounting for limitations on the model accuracy and sensor measurement errors.

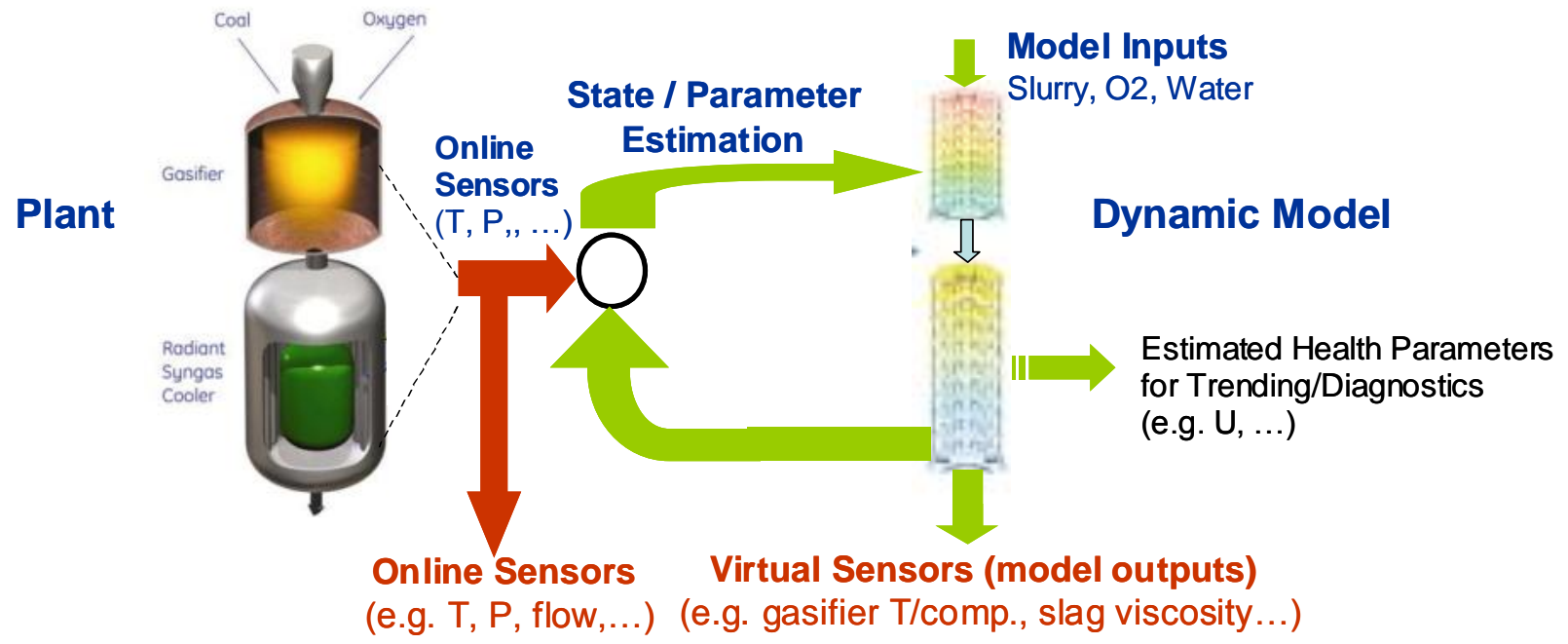

Figure 39: Advanced sensing system for gasification section.

\subsection{Model-based analysis for sensing and estimation}

Consider the state-space model of the gasification section as an ODE system of the form:

Eq 2

$$
\dot{x}=f(x, u, p)
$$

$$
\begin{aligned}
y_{m} & =h_{m}(x, u, p) \\
y & =h(x, u, p)
\end{aligned}
$$

where $x$ is the vector of states, $u$ is the vector of process inputs (control inputs and disturbance inputs, e.g. slurry flow rate and temperature), $p$ is the vector of process model parameters (e.g. gasifier kinetics, fouling factor), $y_{m}$ is the vector of measured outputs (online sensors), and $y$ is the vector of outputs needed for monitoring and controlled (including some that may be measured and some that are not). The corresponding linearized model state-space representation is:

\section{Eq 3}

$$
\dot{x}=A x+B_{u} u+B_{p} p
$$

$$
\begin{aligned}
y_{m} & =C^{m} x+D_{u}^{m} u+D_{p}^{m} p \\
y & =C x+D_{u} u+D_{p} p
\end{aligned}
$$

Discretizing the above linear state-space model and including models for process uncertainties, the linear discrete-time model for the system has the form: 


$$
\begin{aligned}
x_{k+1} & =A x_{k}+B_{u} u_{k}+B_{p} p_{k}+w_{x} \\
u_{k+1} & =u_{k}+w_{u} \\
p_{k+1} & =p_{k}+w_{p} \\
b_{y, k+1} & =b_{y, k}+w_{b y} \\
b_{u, k+1} & =b_{u, k}+w_{b u} \\
y_{m, k} & =C^{m} x_{k}+D_{u}^{m} u_{k}+D_{p}^{m} p_{k}+b_{y, k}+v_{y} \\
u_{m, k} & =u_{k}-b_{u, k}+v_{u} \\
y_{k} & =C x_{k}+D_{u} u_{k}+D_{p} p_{k}
\end{aligned}
$$

In the above discrete-time representation, with a slight abuse of notation, the state-space matrices are denoted by the same matrices $A, B_{u}, B_{p}$ as in continuous-time system to keep notation simplicity, although strictly speaking, these are not the same matrices. Also, the process inputs $u_{k}$ and model parameters $p_{k}$ are included in an extended state vector with a random-walk model. The parameters $p_{k}$ are unknown and constant or slowly varying, while the true inputs $u_{k}$ are also not known perfectly due to measurement errors. The variables $w_{x}, w_{u}, w_{p}$ denote the process noise (uncertainty in the model dynamics) assumed to be stationary and white-noise with specified covariances $Q_{x}, Q_{u}, Q_{p}$, respectively. The online measurements for process outputs $y_{m, k}$ and inputs $u_{m, k}$ have unknown biases $b_{y, k}$ and $b_{u, k}$ as well as measurement noise $v_{y}$ and $v_{u}$, respectively. The measurement noise are assumed to be uncorrelated white noise with covariances $R_{y}$ and $R_{u}$, while the unknown constant/slowly varying measurement biases are modeled as random walk with process noise covariances $Q_{b y}$ and $Q_{b u}$, respectively. The above representation denotes a general description with modeling errors (uncertain parameters, uncertain modeling dynamics or process noise) as well as measurement errors (unknown biases and noise). The linear system in Eq 4 can be represented in a compact state-space form with the extended state vector $x^{e}=\left[x ; u ; p ; b_{y} ; b_{u}\right]$ and extended measurement vector $y_{m}{ }^{e}=\left[y_{m} ; u_{m}\right]$

$$
x_{k+1}^{e}=A^{e} x_{k}^{e}+w^{e}
$$

Eq $5 \quad y_{m, k}^{e}=C_{m}^{e} x_{k}^{e}+v_{y}^{e}$, where

$$
\begin{aligned}
& y_{k}^{e}=C^{e} x_{k}^{e} \\
& A^{e}=\left[\begin{array}{ccccc}
A & B_{u} & B_{p} & 0 & 0 \\
0 & I & 0 & 0 & 0 \\
0 & 0 & I & 0 & 0 \\
0 & 0 & 0 & I & 0 \\
0 & 0 & 0 & 0 & I
\end{array}\right], C_{m}^{e}=\left[\begin{array}{ccccc}
C^{m} & D_{u}^{m} & D_{p}^{m} & I & 0 \\
0 & I & 0 & 0 & -I
\end{array}\right] \\
& C^{e}=\left[\begin{array}{lllll}
C & D_{u} & D_{p} & 0 & 0
\end{array}\right]
\end{aligned}
$$

with corresponding extended process and measurement noise covariances $Q^{e}$ and $R^{e}$, respectively.

Based on the linear extended system description in Eq 5, a Kalman filter analysis can be performed for a given sensor set $\left(y_{m}, u_{m}\right)$ with specified measurement errors to obtain the accuracy in estimated outputs $y$ that are important for monitoring and control. More specifically, the covariances in the estimated outputs are used to quantify the expected uncertainty in the estimated variables. Figure 40 shows the estimation error evolution over 
multiple samples of estimation for four key unmeasured variables that are critical for gasification section monitoring and control. In particular, for each variable, the red plots show the $1-\sigma$ uncertainty in the estimated variable in the presence of process model errors and measurement noise only, while the blue plots show the increased uncertainty due to the added unknown measurement biases in all measurements (the 1- $\sigma$ uncertainty is obtained as the square-root of the corresponding covariance, i.e. $\sqrt{C_{i}^{e} P_{k \mid k}\left(C_{i}^{e}\right)^{T}}$ for the $i^{\text {th }}$ output $y_{i}{ }_{i}$ ). Note that the inclusion of the sensor biases leads to a significant increase in the estimation inaccuracy for the four variables, i.e., increased variances, implying the importance of sensor biases in the overall sensing system performance. Moreover, a significant increase in estimation inaccuracy due to sensor biases indicates that the overall estimation performance can be improved by improving the accuracy of the online sensors - either by using more accurate (which often means more expensive) sensors or using multiple sensors and averaging to reduce the effect of unknown measurement biases. Often, multiple online sensors are already used for increased redundancy/reliability for critical variables.
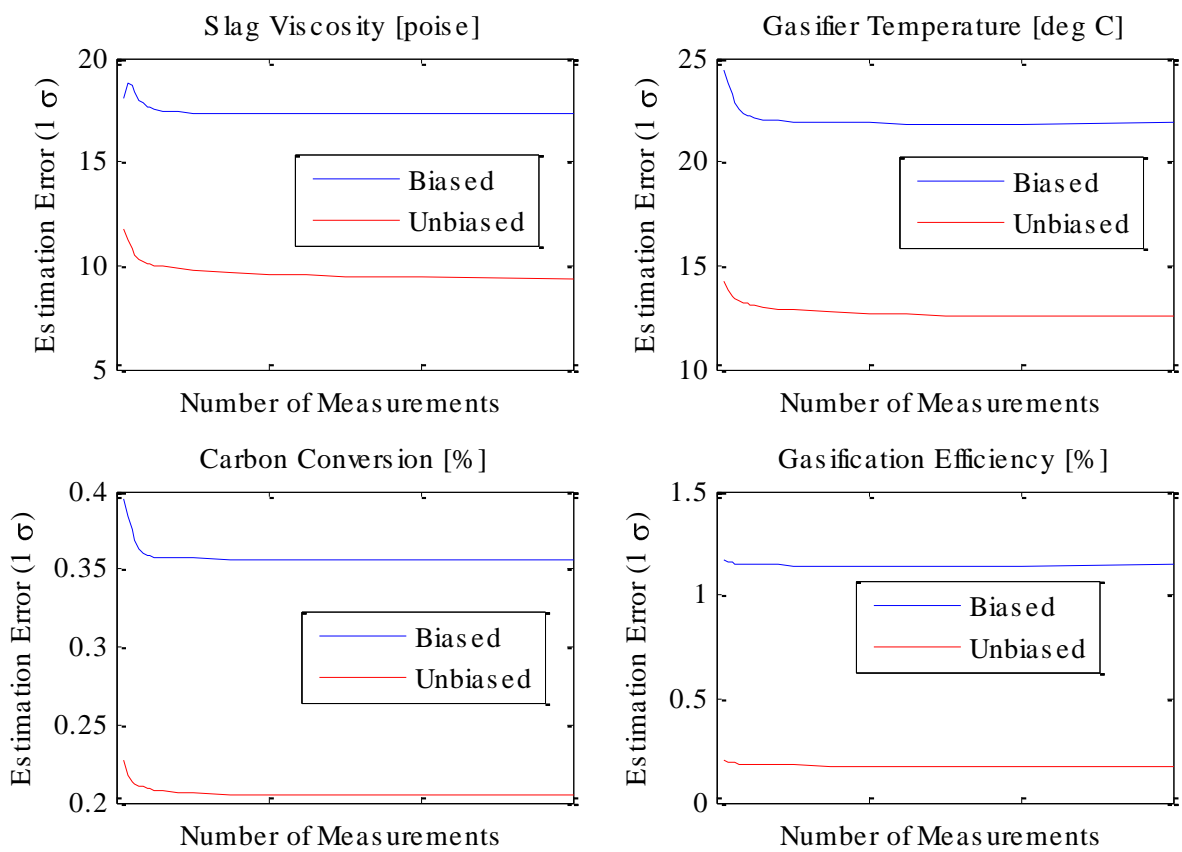

Figure 40: Estimation errors for important un-measured variables due to modeling error and with/without sensor measurement errors.

Figure 41 shows the estimation errors in the same variables due to modeling errors and sensor measurement errors in blue (same as in Figure 40), compared with estimation errors in the presence of only sensor measurement errors with a perfect model in red. Again, the difference between the red and blue plots shows the impact of modeling errors on the estimation accuracy. Comparing the plots in Figure 40 and Figure 41, it is evident in this case that sensor measurement errors - noise and bias are a more dominant source of estimation error. 

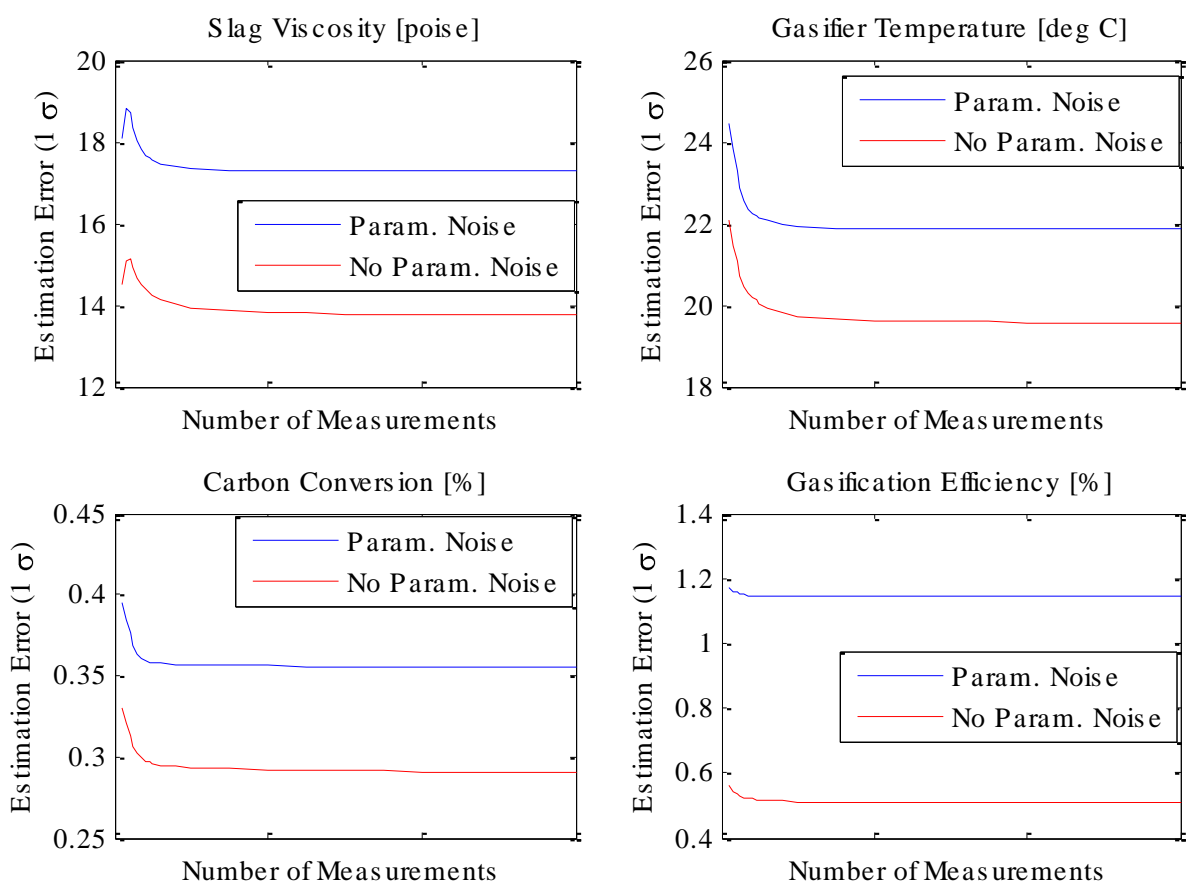

Figure 41: Estimation errors for important un-measured variables due to sensor measurement error and with/without modeling errors.

In 2009 Q1, the focus of the sensing design studies was identifying the most important biases and parameters that were responsible for most of the depreciation of the estimation performance. First, the sensitivity of estimation errors to sensor biases was analyzed by eliminating sensor biases, one sensor at a time, and observing the improvement in the estimation errors of slag viscosity, gasifier temperature, carbon conversion, and gasification efficiency. This sensitivity analysis allowed identifying the critical sensor biases to improve upon (either through improved technology or through sensor redundancy) for a given set of sensors. Similarly, a sensitivity analysis with respect to process model parameters was done by eliminating errors in one parameter at a time to identify key process model parameters whose uncertainty contributes significantly to the estimation inaccuracy of the key variables. In the following section, the results of the sensitivity studies for gasifier temperature estimation are presented as an example of how the analysis can be used to highlight the critical sensor biases and parameters in the model that should be estimated online to improve overall estimation performance. 
Gasifier Exit Syngas Temperature

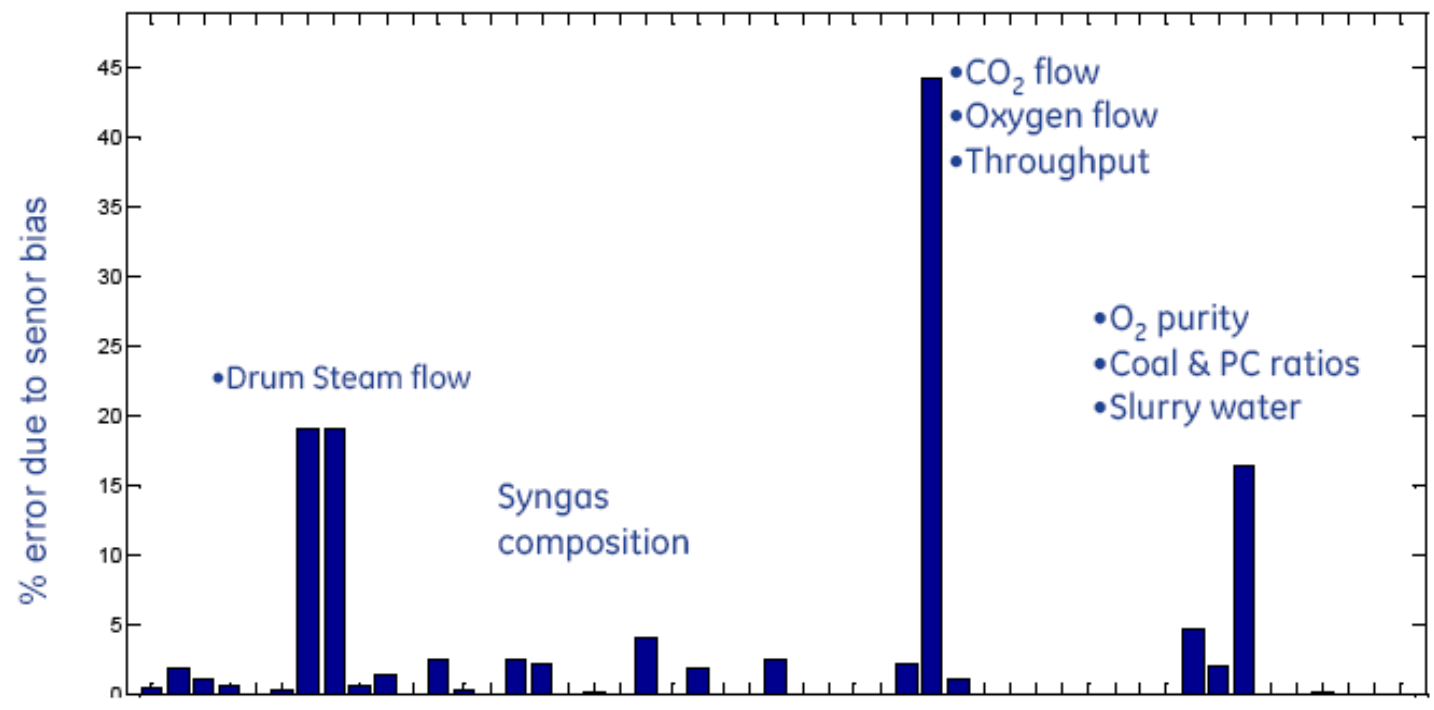

Figure 42: The sensitivity of the estimation error of gasifier exit syngas temperature to the biases of the baseline sensor set. The important biases are drum steam flow, oxygen flow, $\mathrm{CO}_{2}$ flow, throughput, $\mathrm{O}_{2}$ flow and purity, and fuel composition.

Figure 42 shows the sensitivity of estimation error in gasifier temperature to the biases in the IGCC baseline sensor set. The metric for sensitivity is the percent reduction of the estimation error when a specific sensor bias is removed (i.e. the associated sensor is improved to have zero bias). Clearly, the error in estimation of the syngas temperature at the exit of the gasifier is very sensitive to the sensor-biases of the manipulated variables that affect the $\mathrm{O} / \mathrm{C}$ ratio in the gasifier, e.g., oxygen flow, $\mathrm{CO}_{2}$ flow, slurry throughput, oxygen purity, fuel composition. As $\mathrm{O} / \mathrm{C}$ ratio has a direct effect on the gasifier temperature, the high sensitivities to these biases are expected. However, the sensitivity to the drum steam flow sensor-bias is not that apparent. The Kalman filter estimates the temperature of the syngas from the gasifier by utilizing the mass and energy balances around the syngas in RSC, quench, and the gasifier. The drum steam flow measurement is clearly important for the energy balance, and thus, the bias on drum steam flow measurement has a significant effect on the estimation error of the gasifier exit syngas temperature. 


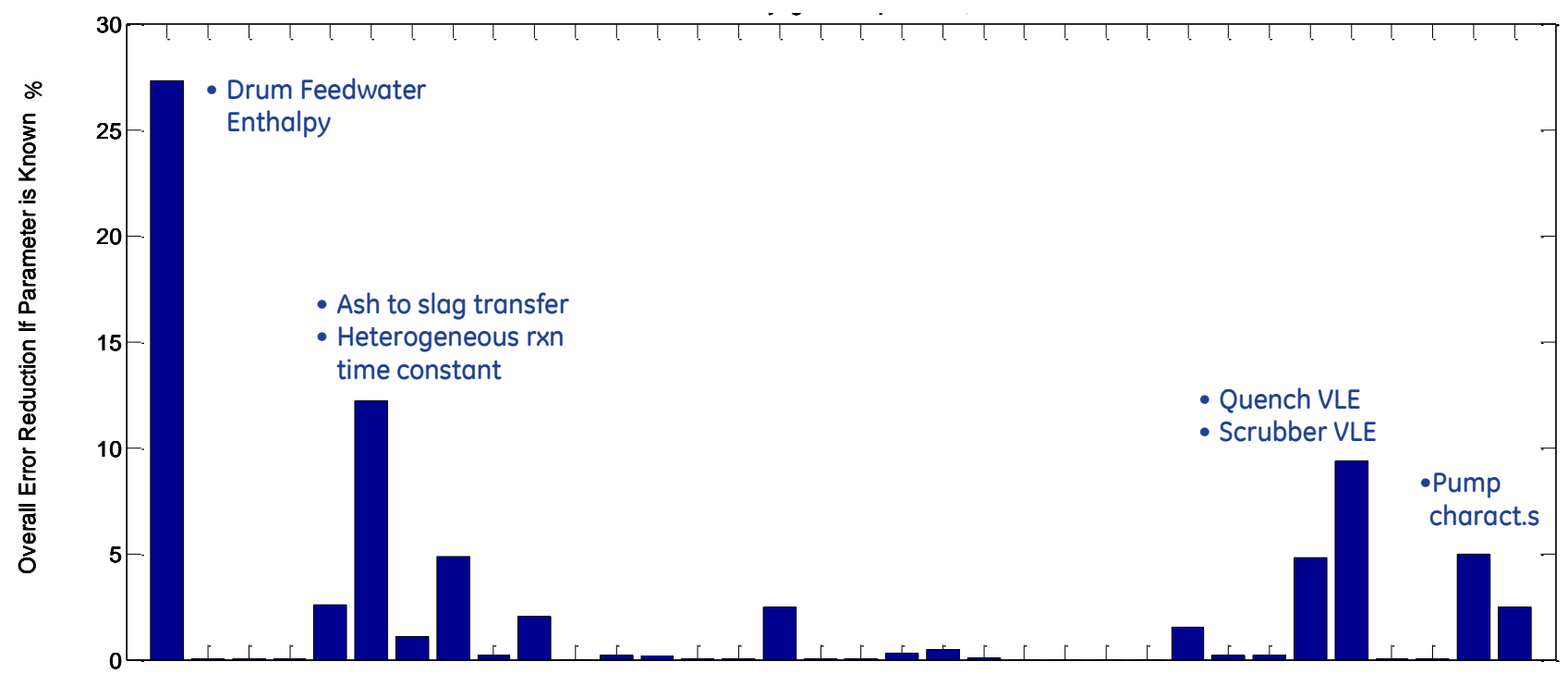

Figure 43: The sensitivity of the estimation error of gasifier exit syngas temperature to the model parameters. The important parameters are drum feed water enthalpy, ash-to-slag transfer rate, heterogeneous reaction time constant, quench and scrubber vapor-liquid equilibrium temperature mismatches, and drum circulation pump's head/flow characteristics.

Figure 43 shows the observed percent improvements in the estimation of the gasifier temperature when uncertainties in the model parameters are removed one at a time. It is evident that when certain parameters are known perfectly (i.e. there is no associated uncertainty for these parameters), the estimation error in the gasifier temperature (1- $\sigma$ error) improves significantly. As discussed in the analysis of the bias sensitivity study, the Kalman filter relies on the energy balance around the gasification section to infer the gasifier temperature. For this energy balance, the steam production inside the drum is important since it indicates the amount of heat removed from the syngas in the RSC. The parameter, drum feed water enthalpy, is important for the same reason - one can understand how much enthalpy was introduced to the drum with the feed water through this parameter. The ash to slag transfer parameter determines how much ash is transferred to the slag from the coal/syngas and therefore is an indicator of how much heat is lost through the slag. The heterogeneous reaction time constant dictates the extent of the endothermic/exothermic reactions inside the gasifier and therefore affects the overall mass/energy balance and the resulting estimation error significantly. The baseline quench and scrubber models assume that the vapor (syngas) and liquid (water) phases are in thermodynamic equilibrium, which is described through a non-ideal vapor liquid equilibrium (VLE). However, in the true plant the vapor and liquid phases may not necessarily be in equilibrium and/or the non-ideal VLE model may be inaccurate. These errors are incorporated in the model through deviation in the temperature used for VLE calculations, both in the quench and the scrubber. The last two parameters, to which the gasification temperature estimation is sensitive, are related to the pump characteristics scale factors. These scale factors determine the circulation of water between the drum and the RSC and consequently affect the energy balance of heat removal in RSC. Hence, they play an important role in the estimation of gasifier temperature from downstream measurements and the system model. 
The results of the above linear model-based Kalman filter analysis was used to design a nonlinear model-based estimation using Extended Kalman Filter (EKF) incorporating estimation of the key sensor biases and model parameters showing high sensitivity to estimation accuracy.

\subsection{Extended Kalman Filter Simulation Studies for Model-based Estimation}

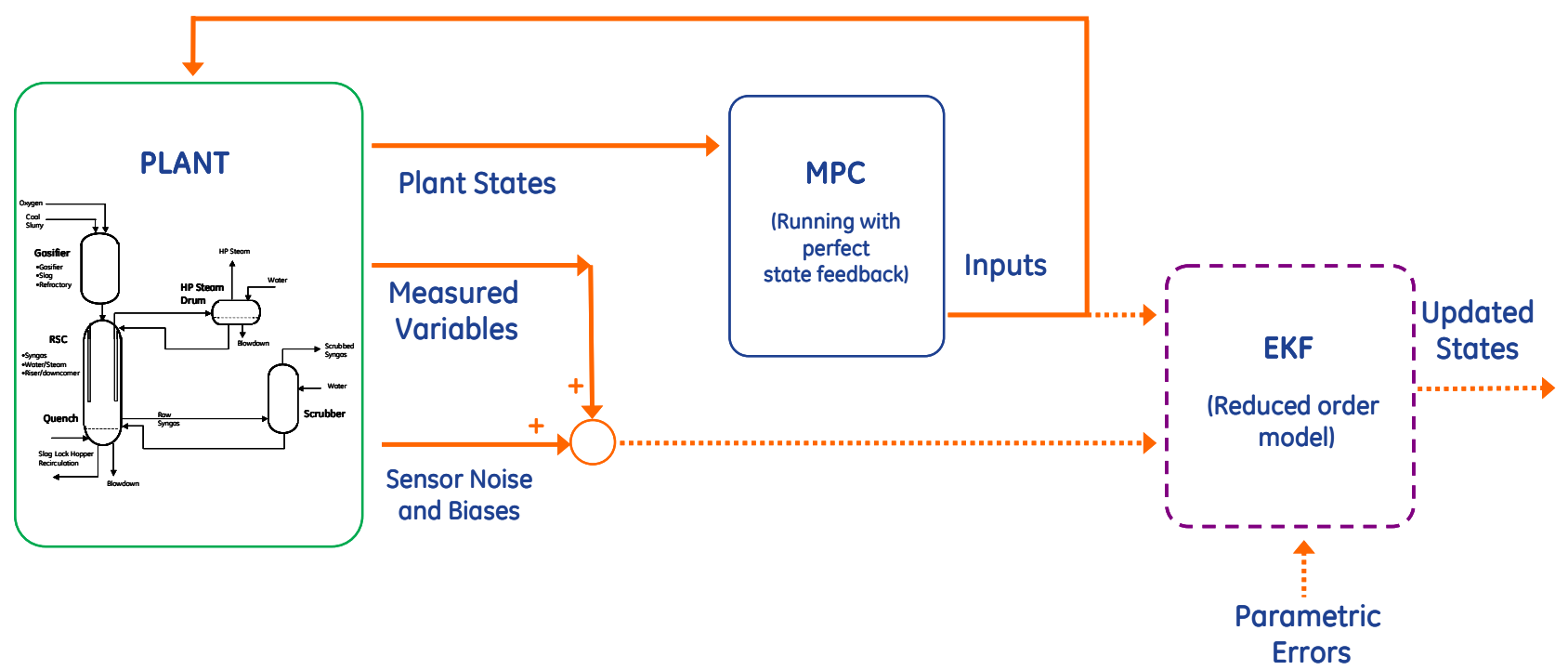

Figure 44: Open-loop implementation of the extended Kalman filter, where EKF is running in parallel to the MPC with state feedback from the plant.

Following up on the linear model-based Kalman filter analysis for sensing system performance in the presence of sensing and modeling errors, a nonlinear model-based estimation algorithm was implemented in Matlab/Simulink® to complement the online sensors and obtain the overall sensing system shown in Figure 39. More specifically, an Extended Kalman Filter (EKF) was implemented to perform the nonlinear model-based estimation. EKF is a standard model-based estimation algorithm that allows an "optimal least-squares" model-based estimation of unknown process model states utilizing available online measurements, while optimally trading off the errors in the dynamic model and online sensor measurements. In Phase II of the program, the EKF was initially developed and tested in open-loop configuration as shown in Figure 44, i.e., it was run in parallel to the MPC implementation. The developed EKF and MPC solutions were then coupled in Phase III of the program to derive the overall integrated sensing and control solution. Initially, the EKF was simulated with no sensor or modeling errors to verify its stability and performance. It should be mentioned that in all simulations, the full-order detailed model was used to simulate the plant. However, this model is stiff and forces the use of very small integration step size in a fixed step-size integration scheme. The EKF uses a reduced-order model as the embedded model during the propagation step, which is obtained by approximating fast dynamics related to pressure drop-flow relations for the syngas with corresponding quasi-steady-state conditions. This is the akin to the use of the reduced-order non-stiff model for the MPC, allowing the use of larger integration step-size, and thus, reduced computational cost during MPC prediction. Note that the linear model- 
based analysis was performed using the full-order stiff model, which includes the fast dynamics corresponding to syngas flow and pressure. Thus, the overall gasification section efficiency showed high estimation inaccuracies (Figure 40, Figure 41) since the efficiency depends on estimated syngas flow rate, which in turn, is very sensitive to small measurement errors in gasifier, quench and scrubber pressures. The reduced-order model in the EKF implementation overcomes this undue sensitivity and yields higher accuracy in efficiency as shown later in this section.

The EKF implementation for the nonlinear system with extended state vector $x^{e}$ is:

$$
\left.\begin{array}{c}
\hat{x}_{k+1 \mid k}^{e}=f\left(\hat{x}_{k \mid k}^{e}\right) \\
P_{k+1 \mid k}=A^{e} P_{k \mid k} A^{e^{T}}+Q^{e}
\end{array}\right\} \text { propagate, starting with } \hat{x}_{0}^{e}, P_{0 \mid 0}
$$

Eq 6

$$
\left.\begin{array}{c}
\hat{x}_{k+1 \mid k+1}^{e}=\hat{x}_{k+1 \mid k}^{e}+K_{k}\left(y_{m, k}^{e}-C_{m}^{e} \hat{x}_{k+1 \mid k}^{e}\right) \\
K_{k}=P_{k+1 \mid k} C_{m}^{e^{T}}\left(C_{m}^{e} P_{k+1 \mid k} C_{m}^{e^{T}}+R^{e}\right)^{-1} \\
P_{k+1 \mid k+1}=\left(I-K_{k} C_{m}^{e}\right) P_{k+1 \mid k}\left(I-K_{k} C_{m}^{e}\right)^{T}+K_{k} R^{e} K_{k}^{T}
\end{array}\right\} \text { measurement update }
$$

The first step (propagate) is the simulation of the process model forward in time to update the state estimates from one time sample $k$ to the next sample $k+1$, and the corresponding covariance update with the linearized model. The second step is the measurement update, where the error between the measured outputs $y_{m, k}^{e}$ and the predicted output values $\hat{y}_{m, k}^{e}=C_{m}^{e} \hat{x}_{k+1 \mid k}^{e}$ is used to correct the state estimate through the Kalman filter gain $K_{k}$. The gain $K_{k}$ is calculated recursively at each time step $k$, as the solution to the leastsquares estimation in the presence of the variances $Q^{e}$ for the process model uncertainty and $R^{e}$ for the measurement errors. One issue with the standard EKF is that there is no means to enforce physical limits/constraints on estimated state variables. For instance, the state vector $\hat{x}_{k \mid k}^{e}$ includes mole fractions that should be in the range $0-1$ to be meaningful. Similarly, model parameters being estimated include RSC fouling that should also be in the range 0-1 ( 0 being new RSC and 1 being fully fouled RSC). The standard EKF can violate these constraints, especially during the measurement update step if the estimator gain $K_{k}$ is high. A violation of these constraints leads to failure in exercising the physicsbased model.

To address this issue, the standard EKF implementation was modified to include provision for verifying any constraints that would be violated during a standard measurement update step, and thus should be made "active" or enforced at an active boundary. More specifically, considering a set of constraints on the extended state variables of the form

$$
\text { Eq } 7 \quad C^{c} x^{e} \leq D^{c} \text {, }
$$

the measurement update step is extended to detect for any "active" constraints and modify the state and covariance update. This was done by first identifying the set of "active" constraints that must be enforced as equality constraints, through a simple quadratic programming $(\mathrm{QP})$ formulation:

Eq 8

$$
\begin{aligned}
& \min _{\tilde{x}_{k+1 \mid k+1}}\left(\tilde{x}_{k+1 \mid k+1}^{e}-\hat{x}_{k+1 \mid k+1}^{e}\right)^{T} W\left(\tilde{x}_{k+1 \mid k+1}^{e}-\hat{x}_{k+1 \mid k+1}^{e}\right) \\
& C^{c} \tilde{x}_{k+1 \mid k+1}^{e} \leq D^{c}
\end{aligned}
$$


In the above QP problem, $W$ is a weighting matrix that was chosen to be the Identity matrix. Essentially, the QP problem was formulated to identify a corrected state estimate that is least distance away from the unconstrained state estimate, and enforces all the inequality constraints. In particular, the QP solution identifies the subset of the constraints that are active and should be enforced as equality constraints:

Eq $9 \quad C_{a}^{c} \tilde{x}_{k+1 \mid k+1}^{e}=D_{a}^{c}$

Once the set of active constraints is identified, a corrected state estimate is identified consistent with these constraints, and the corresponding state covariance is also updated:

$$
\tilde{x}_{k+1 \mid k+1}=K_{k}^{c}\left(D_{a}^{c}-C_{a}^{c} \tilde{x}_{k+1 \mid k+1}^{e}\right)
$$

Eq $10 \quad K_{k}^{c}=P_{k+1 \mid k+1} C_{a}^{c^{T}}\left(C_{a}^{c} P_{k+1 \mid k+1} C_{a}^{c^{T}}\right)^{-1}$

$$
\tilde{P}_{k+1 \mid k+1}=\left(I-K_{k}^{c} C_{a}^{c}\right) P_{k+1 \mid k+1}\left(I-K_{k}^{c} C_{a}^{c}\right)^{T}
$$

Note that the above correction step to enforce active constraints essentially is equivalent to performing a secondary measurement update with respect to the active constraints as "dummy" measured outputs, with a zero measurement noise. The updated state estimate $\tilde{x}_{k+1 \mid k+1}$ enforces the active constraints and is used as the starting point at next sample for the propagate step. Having a zero measurement noise on these "dummy" measurement outputs yields high gain $K_{k}^{c}$, which in turn, can often lead to issues with the estimation performance. To alleviate this, a small "dummy" measurement noise is added to soften the constraint a little. The inclusion of the state constraints on the standard EKF allows enforcing desired physical limits on the estimated state variables (including states, inputs, parameters and measurement biases). This updated EKF with constraints was implemented in Matlab/Simulink® and simulated for steady state and transient operation in the presence of parametric errors in the model. The process and measurement noise covariances were fine-tuned to obtain good transient estimation performance.

The constrained EKF implementation in Simulink was initially tested for its performance in terms of estimation of key important unmeasured variables, in the presence of modeling errors but no sensor biases. The modeling errors were introduced through unknown changes in model parameters like RSC fouling and gasifier reaction kinetics. More specifically, the gasifier kinetics scale factor was changed from nominal value of 1.0 to 0.7 (i.e. make the gasification reactions $30 \%$ slower) and the RSC fouling scale factor was changed from nominal value of 0 (new RSC) to 0.3 (30\% fouling). The model used for the EKF was initialized with the nominal value of these parameters, and the EKF was configured to simultaneously estimate all the unknown parameters and sensor biases while tracking the measurements from the online sensors. In these simulation runs, the sensors did not have any measurement noise or bias - nevertheless the EKF was still configured to estimate the biases since they are not known to be zero.

Figure 45 shows the comparison of four performance/constraint variables important for control that are not measured online, during a transient with changes in load between baseload and $50 \%$ load. The red plots show the true values from the plant simulation (with the modified parameters), the dashed black plots show the predicted outputs from the embedded model used in EKF with nominal values of the parameters (i.e., an open-loop model with no corrections), while the blue plots show the updated estimates as the EKF 
adapts the values of the unknown model parameters. Note that after an initial transient, the EKF provides an accurate estimate of all the variables matching the true plant values throughout the load transients. These four variables are only some of the key unmeasured variables that are important for monitoring and control. The EKF provides similarly accurate estimate for other important variables as well, e.g., syngas properties like LHV and modified Wobbe index (MWI) and oxygen-to-carbon ratio - plots for those are omitted for brevity.

Figure 46 shows a comparison of the true values (red) of the model parameters and EKF estimated values (blue) of these parameters during the load transient. Note that starting from the nominal values of the parameters, the EKF estimation correctly estimates the changed values of the RSC fouling and gasifier kinetics scale factor quite accurately. On the other hand, other parameters like water gas shift (WGS) reaction kinetics scale factor in the RSC are correctly maintained close to the nominal values - note that the WGS kinetics scale factor has a max limit at 1.0, which is maintained by the constrained EKF estimation at all times. This ability to track unknown or varying parameters in the process (e.g. RSC fouling) allows continuously adapting the model to match the online measurements, and thus, providing an accurate estimate of unmeasured variables. Also, the ability to track varying parameters like RSC fouling provides a direct means of online monitoring for equipment health degradation.
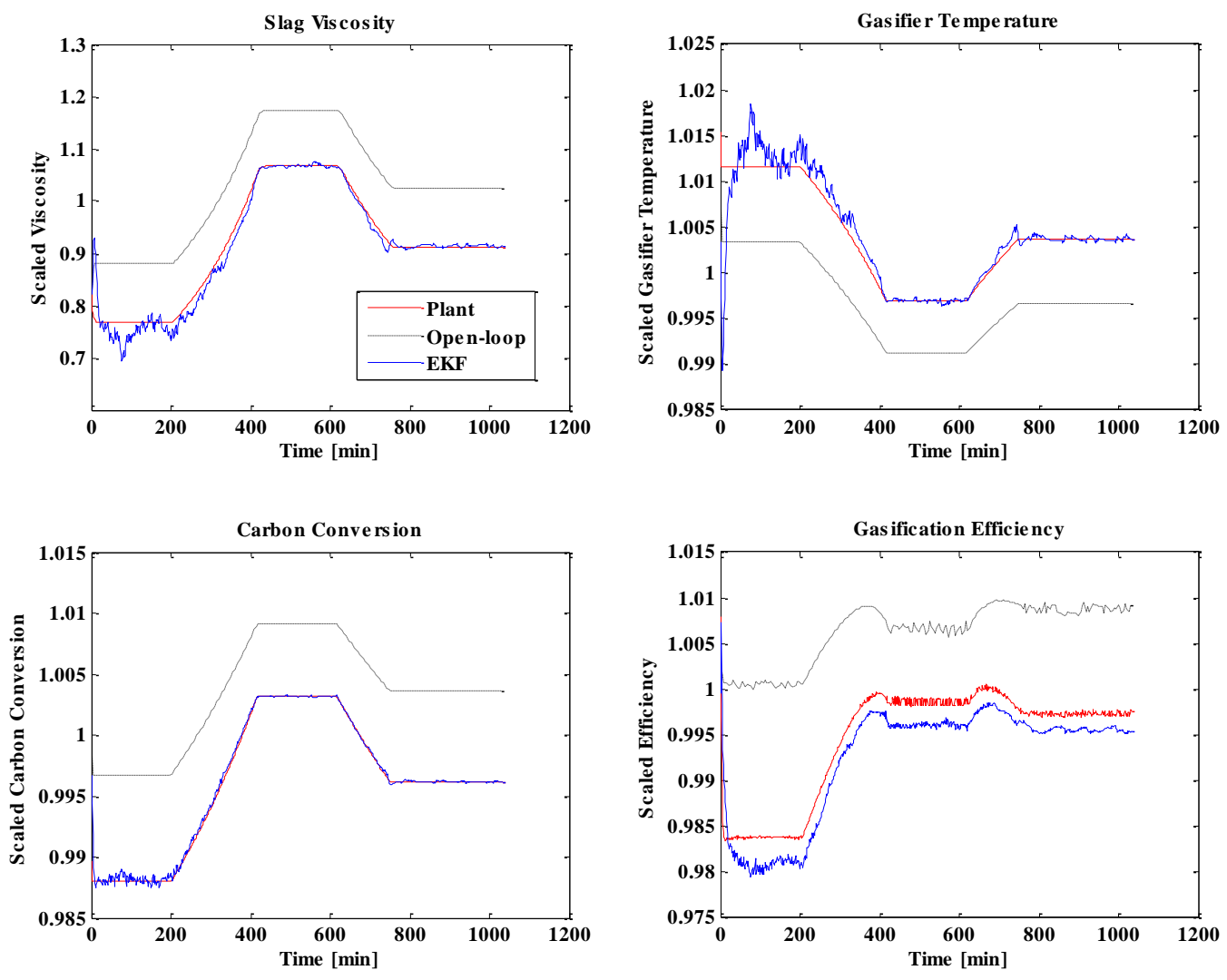

Figure 45: Estimation of key unmeasured process variables during load changes. 

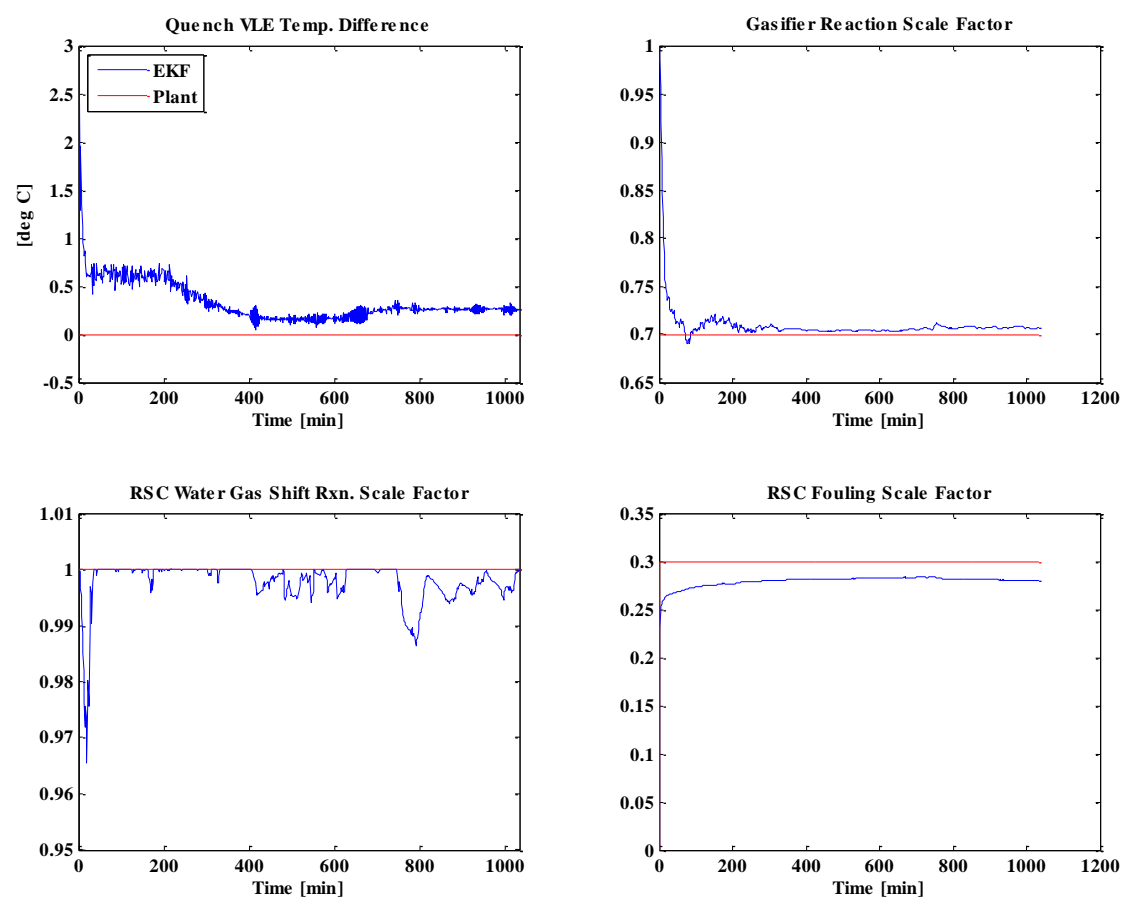

Figure 46: Estimation of unknown model parameters during load changes.

The performance of the EKF estimation was tested for the same unknown changes in RSC fouling and gasifier reaction kinetics, in the presence of varying oxygen feed, or equivalently oxygen-to-carbon ratio, a key parameter adjusted by the operator for plant operation. Figure 47 and Figure 48 show the similar comparison of EKF estimated unmeasured key process variables and unknown model parameters. Again, the EKF correctly updates the estimate for the RSC fouling and gasifier kinetics, and thus, provides an accurate estimate of the unmeasured important process variables.
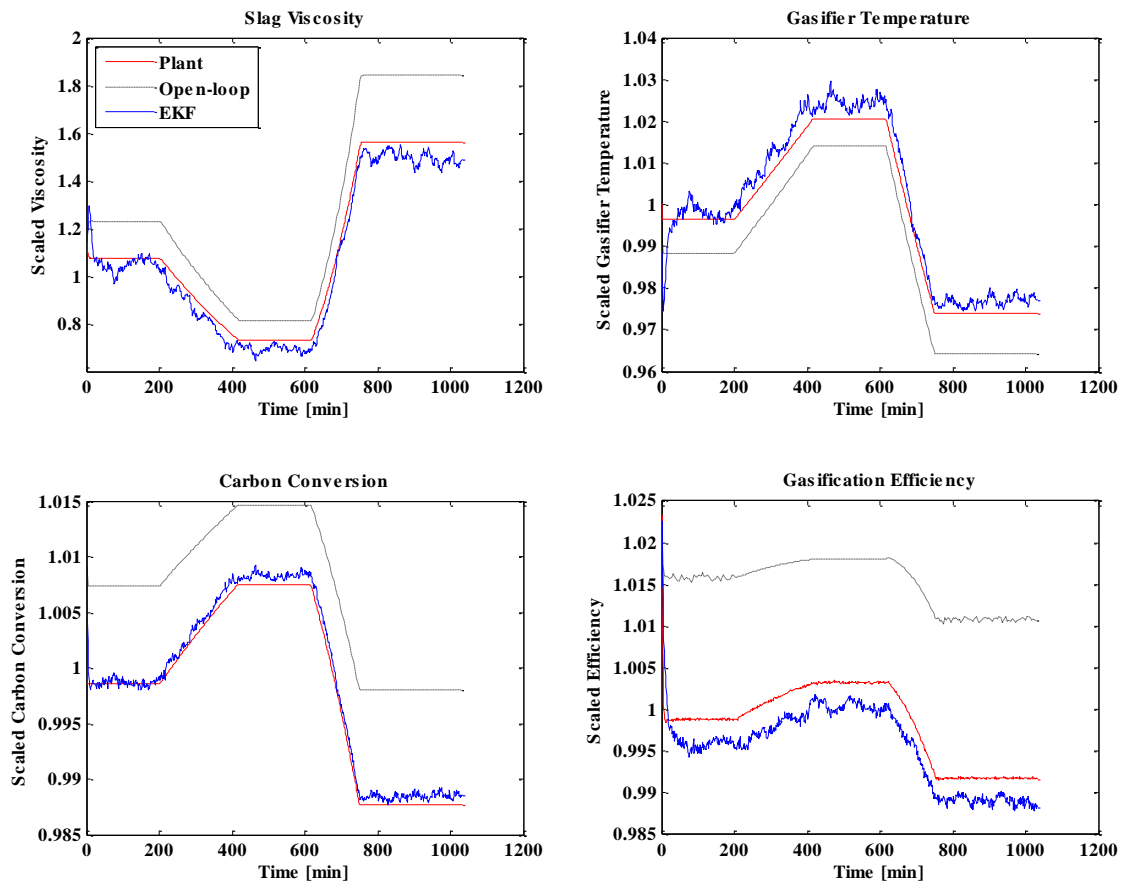

Figure 47: Estimation of key unmeasured process variables during oxygen feed changes. 

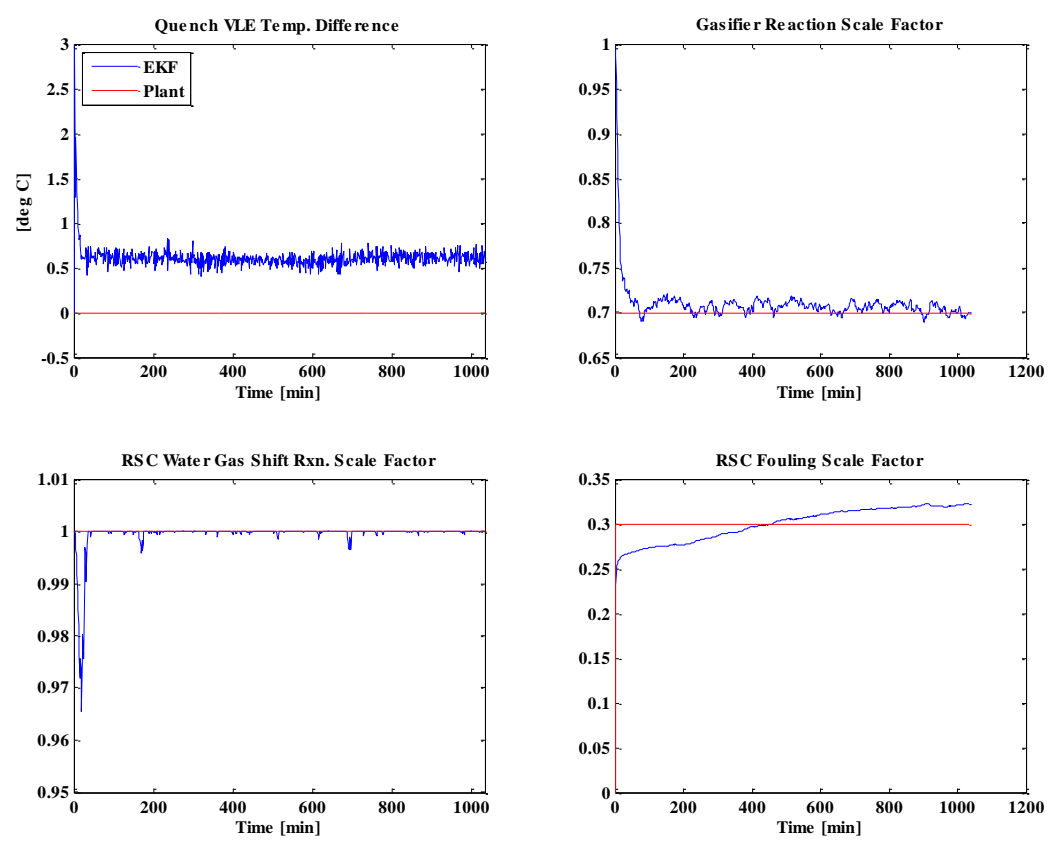

Figure 48: Estimation of unknown model parameters during changes in oxygen feed.

The above studies were performed in the absence of any sensor biases, to establish the performance of the EKF under these conditions. However, in reality, all sensors have unknown measurement accuracy errors or bias typically characterized by either an absolute bound or a 1- $\sigma$ value for expected distribution across multiple sensors of the same type. These biases may be constant or drift slowly over long periods of operation. After the initial studies with EKF simulation in the absence of sensor bias, subsequent studies were performed in the presence of random combinations of sensor biases based on expected 1- $\sigma$ variations for each sensed variable. The EKF implementation was further tuned for a robust performance in the presence of random sensor biases and a small Monte Carlo study was performed with unknown random sensor biases to analyze the performance of the EKF in terms of variation in the estimation accuracy of key unmeasured variables important for monitoring \& control.

During these Monte Carlo runs, limitations were observed in the performance of the EKF in the presence of the inequality constraints on the states being estimated. Specifically, when a parameter/state constraint became active, the corresponding state covariance was collapsed along the directionality of the active parameter/state constraint, which led to parameter/states getting stuck at their respective constraints. This deficiency was alleviated to some extent through a small noise added to the "dummy" measurements used to implement the active constraints, but its performance was still not satisfactory. To address this, a new methodology for implementing inequality constraints, that borrowed ideas from unscented Kalman filtering (UKF), was developed. The methodology relies on the UKF approach to identify the sigma points consistent with the current state covariances. The sigma points that violate any state inequality constraints are then moved to the constraint boundaries and the new state covariance is re-computed with the updated sigma points. In contrast with the post-measurement-update constraint correction used previously, this methodology imposes the state constraints by changing the covariances before the standard measurement update step, i.e., a preemptive constraint 
correction. The development of this algorithm presented an addition to the variety of options for implementing the state constraints in the state-estimation problem: clamping the state estimates, using the post-measurement-updated constrained EKF (cEKF) formulation, the UKF-based pre-emptive constrained formulation, or a combination of these methods. These different approaches were compared in 2009 Q4 by testing their average performances over a benchmark set of Monte Carlo runs. This set of test runs consisted of 62 different simulations with random biases on measurements of manipulated variables, outputs, and disturbances. The methods that were compared included cEKF (with no noise for dummy measurements), cEKF with the small noise added for the dummy measurements for the active constraints (cEKF w. R), clamping the EKF state estimates (clamped EKF), and the UKF-based approach (Preempt. EKF). The performances of the various algorithms are summarized in Table 3.

Table 3: Mean estimation errors of estimation algorithms for benchmark test cases.

\begin{tabular}{|c|c|c|c|c|c|c|c|}
\hline Variable & $\begin{array}{l}\text { Open } \\
\text { Loop }\end{array}$ & cEKF & $\begin{array}{l}\text { cEKF } \\
\text { w. R }\end{array}$ & $\begin{array}{l}\text { Clamped } \\
\text { EKF }\end{array}$ & $\begin{array}{l}\text { Clamped } \\
\text { EKF - no } \\
\text { bias est. }\end{array}$ & $\begin{array}{l}\text { Preempt } \\
\text { EKF }\end{array}$ & \begin{tabular}{|l} 
Preempt \\
EKF - no \\
bias est.
\end{tabular} \\
\hline Slag Visc. & -32.4 & -21.8 & -10.7 & -6.05 & -12.7 & -6.63 & -12.7 \\
\hline $\begin{array}{l}\text { Gasifier } \\
\text { Temp. }\end{array}$ & 16.4 & 10.0 & 5.3 & 2.5 & 6.1 & 2.7 & 6.1 \\
\hline Carb. Conv. & -0.67 & -0.80 & -0.18 & 0.11 & 0.20 & 0.11 & 0.20 \\
\hline Efficiency & -0.70 & -0.37 & 0.05 & 0.1 & -0.15 & 0.1 & -0.15 \\
\hline $\begin{array}{l}\text { Slag } \\
\text { Thickness }\end{array}$ & $-1.6 e-4$ & $-1.1 e-4$ & $-3.1 e-4$ & $-7.9 e-5$ & $-5.2 e-4$ & $-1.1 \mathrm{e}-4$ & $-5.2 e-4$ \\
\hline $\begin{array}{l}\text { O to Carb. } \\
\text { Ratio } \\
\end{array}$ & 0.0058 & 0.0045 & 0.0058 & 0.0058 & 0.0064 & 0.0058 & 0.0064 \\
\hline LHV & -1.76 & -1.98 & -1.21 & -0.77 & -0.53 & -0.75 & -0.54 \\
\hline IWI & -0.08 & -0.09 & -0.05 & -0.03 & -0.02 & -0.03 & -0.02 \\
\hline
\end{tabular}

As can be seen from Table 3, the most successful constrained estimation methods are the clamped EKF and the preemptive constrained EKF. The benefit of estimation of the biases was also analyzed by disabling the bias estimation in the two estimation methods, seen in the columns of Table 3 denoted by "no bias est.". Clearly, when the biases were not estimated the performances of these methods degraded, justifying the estimation of the biases for improved performance. The performance of the preemptive constrained EKF over the benchmark test cases and comparisons with open-loop estimation is presented in Figure 49 for the four critical unmeasured variables: slag viscosity, gasifier temperature, carbon conversion, and efficiency. 

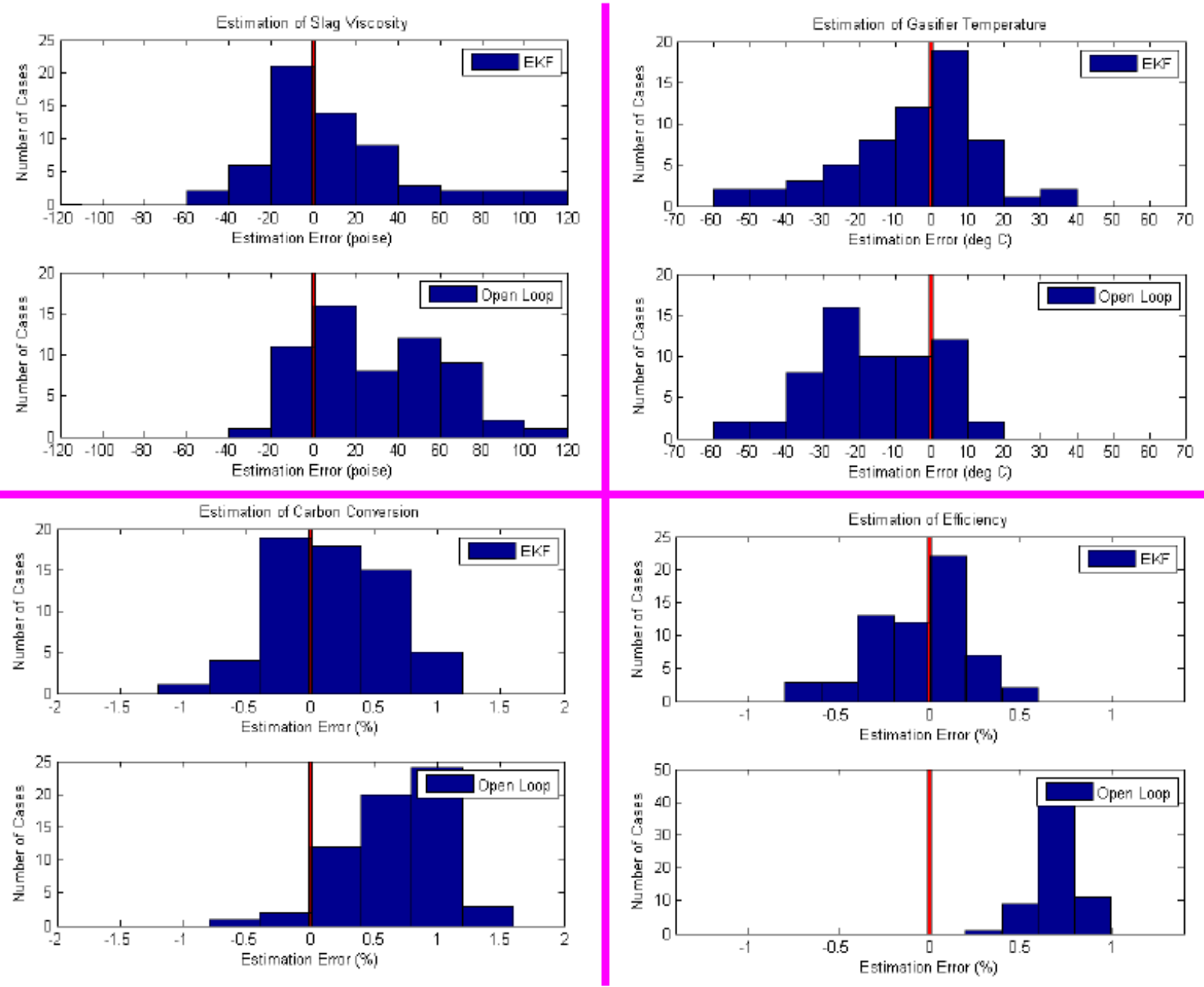

Figure 49: Performance of the preemptive constrained EKF compared with the open-loop estimation for the four critical unmeasured variables. EKF estimate shows close to zeromean while open-loop estimates have significant non-zero mean.

From Figure 49, it is clear that the open-loop estimation of the critical unmeasured variables shows a strong bias (non-zero mean error), while the preemptive constrained EKF shows a significantly superior performance with close to zero average error - the comparison of these estimate mean errors is quantified in Table 3. Note however, that even though the overall and average performance of the preemptive EKF is superior to open-loop estimation, the estimation error distribution for instance for the gasifier temperature shows a long tail corresponding to cases in the Monte Carlo run where the EKF significantly under- or over-predicts the gasifier temperature. This "non-normal" error distribution with long tails prompted further analysis to understand the cause. In particular, the aim was to identify the specific combination of sensor biases that lead to poor estimation performance - the estimator is unable to distinguish individual sensor biases in the case of this combination and thus performs poorly. Picking gasifier temperature as a candidate since the correct estimation of this variable affects estimation of the other variables profoundly (e.g. slag viscosity), the simulations in the benchmark test-case set were identified that had large estimation errors, as presented in Figure 50. 


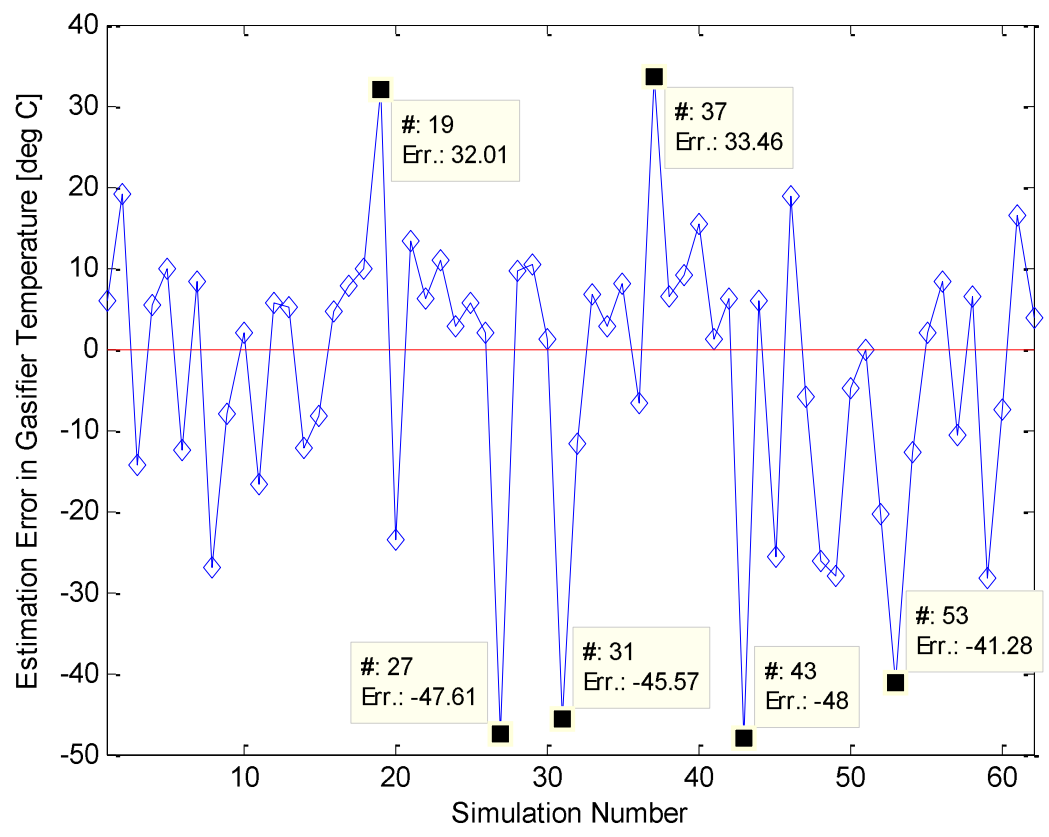

Figure 50: Determination of the outliers in the estimation of the gasifier temperature in the test runs. Each point represents the steady state estimation error of the gasifier temperature in that specific run. Simulation runs 19, 27, 31, 37, 43, and 53 were the outliers.

As shown in Figure 50, the combination of random biases that were implemented in the simulation runs $19,27,31,37,43$, and 53 were found to be causing issues for the accurate estimation of the gasifier temperature. A closer first-look at these outlier cases revealed that the biases on the measurements of gasifier pressure and $\mathrm{O}_{2}$ feed flow rate were particularly high in these runs and the signs of the biases on these cases were correlated with the signs of the estimation errors. A more structured analysis to understand which combination of biases was challenging the estimator was carried by utilizing Partial Least Squares (PLS) regression.

PLS is a technique to identify dominant modes of independent $(X-$ in this case the sensor biases) and dependent ( $Y$ - in this case the gasifier temperature estimation error) data so as to maximize the covariance between the dominant modes of $X$ and the dominant modes of $Y$, i.e. identify the relationship between the variations in $X$ and $Y$. A successful PLS regression often leads to a few modes in $X$, often much lower than the number of variables in $\mathrm{X}$, explaining more than $90 \%$ of the variation observed in a given $\mathrm{Y}$-data. Using the biases on the measured outputs, disturbances, and manipulated variables over the set of test runs as the predictor data $(X)$ and the estimation error observed in gasifier temperature in these runs as the dependent data $(\mathrm{Y})$, a PLS regression was carried out to find the dominant combinations of the biases that were affecting the estimation error of the gasifier temperature. Figure 51 shows the performance of the PLS regression model that correlated the biases during each run in the test cases and the gasifier temperature estimation error in those test cases. As it is clear from the agreement between the PLS predictions and the actual estimation errors, the regression model captured the variance observed in the estimation error data very well. Only 3 dominant modes of $X$ data (biases) were used in the PLS regression model that explained $96 \%$ of the variance observed in the $\mathrm{Y}$ data (estimation error). 


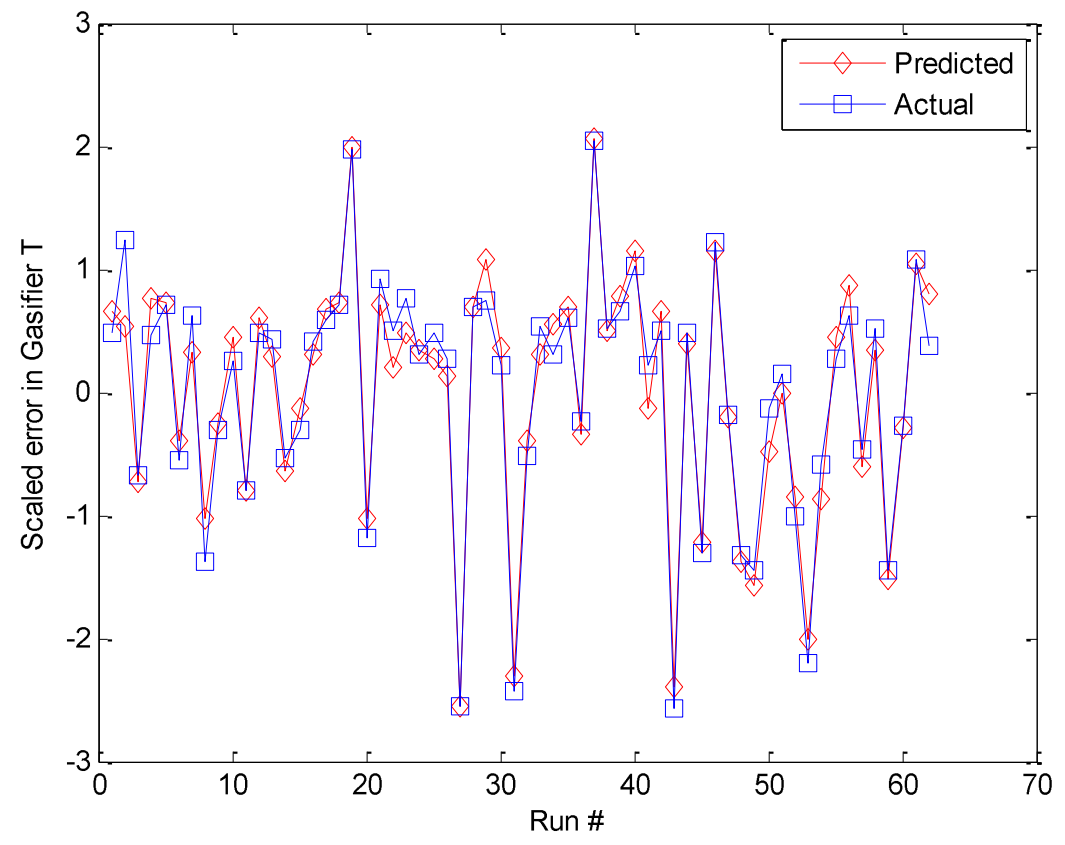

Figure 51: Performance of the PLS regression explaining the relationship between the biases and the gasifier temperature estimation error. The PLS regression model explains more than $95 \%$ of the variance observed in the estimation error data.

Having built a successful correlation between the biases $(X)$ and the gasifier temperature estimation errors $(Y)$, the 3 modes were analyzed to find out which biases contributed most to these modes. Figure 52 shows the cumulative loading of each bias on these dominant modes. The first conclusion that can be drawn from the loadings plot is that the estimation performance of the gasifier temperature is heavily affected by a combination of the biases rather than a single bias. However, it is also apparent that the biases on the gasifier pressure and the $\mathrm{O}_{2}$ flow rate have a more significant impact on the estimation error than the other biases, which was also observed by the unstructured first-look at the outlier cases in the estimation of the gasifier temperature. This analysis indicated that for the specific runs in the Monte Carlo simulation where the $\mathrm{O}_{2}$ flow rate measurement and gasifier pressure measurement, and to a lesser extent other sensor biases like water/steam recirculation flow rate, were large simultaneously, led to estimation performance deterioration. In the presence of simultaneous biases in these sensors, the EKF is unable to distinguish between them and leads to degraded gasifier temperature estimation. To test this conclusion, the Monte Carlo run with the same set of random biases, but with $\mathrm{O}_{2}$ flow rate bias set to zero, was repeated. Figure 53 shows the performance of the preemptive EKF for estimating the gasifier temperature, before and after the elimination of the $\mathrm{O}_{2}$ flow rate bias. It is clear that when the $\mathrm{O}_{2}$ flow rate bias is eliminated, the estimation of the gasifier temperature is improved significantly, as depicted by the disappearance of the tails of the error distribution. Therefore it can be concluded that the elimination or drastic reduction of the measurement bias in the $\mathrm{O}_{2}$ flow rate either through better or redundant instrumentation will be very beneficial for the correct estimation of the temperature inside the gasifier, a critical yet unmeasured process variable. 


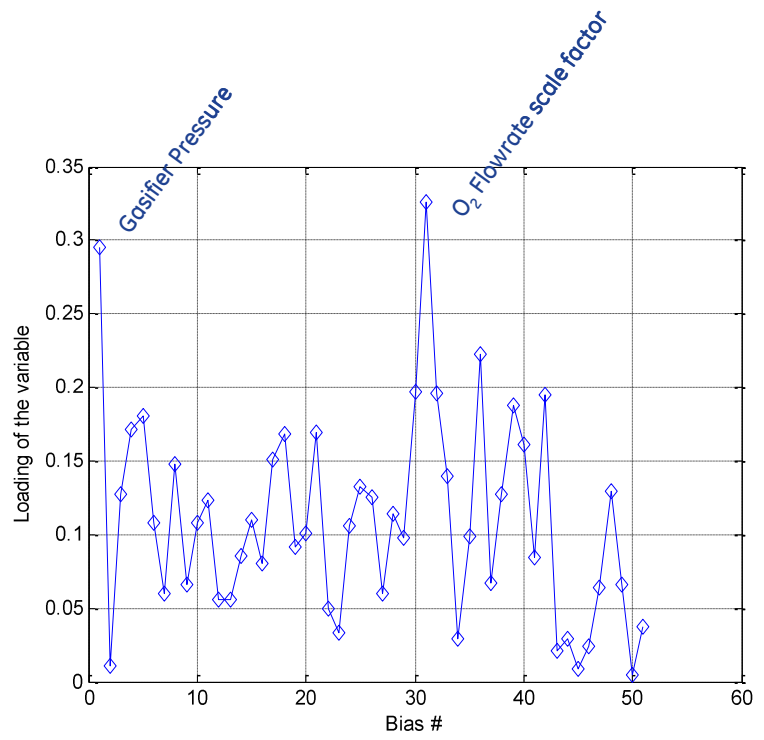

Figure 52: The cumulative loadings of the biases on the first three modes of the PLS regression.
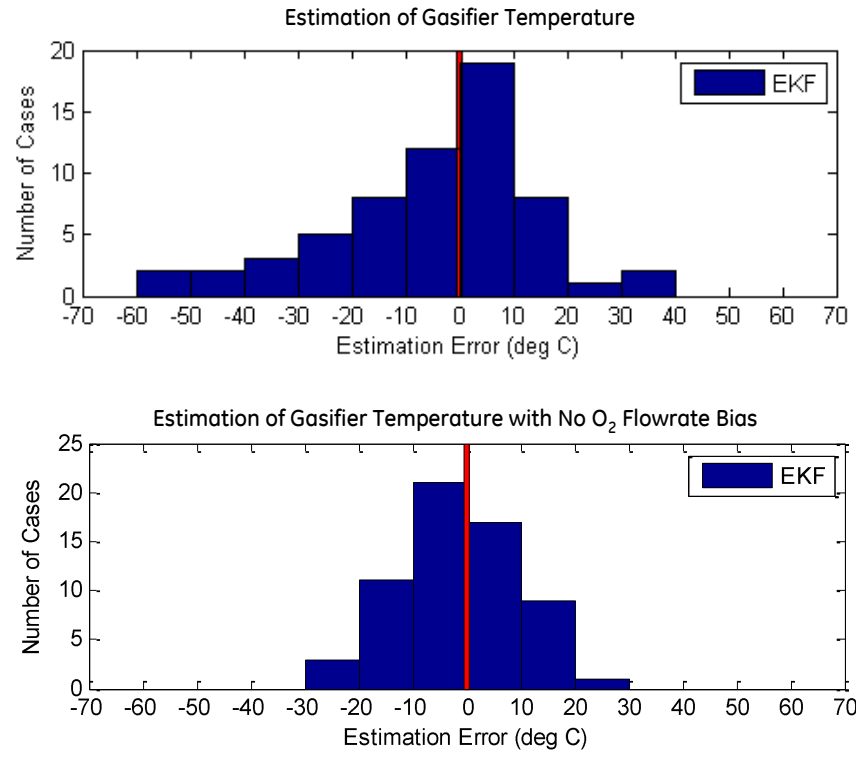

Figure 53: The comparison of the estimation of gasifier temperature in the test cases before and after the elimination of the $\mathrm{O}_{2}$ flow rate bias.

The sensing system combining available online sensors together with a model-based online estimation provides an online real-time feedback of the critical performance and operability variables that are not measured online. This capability is, in itself, very valuable to the plant operator to enable monitoring the actual state of the system continuously and allow more informed decision-making. Integrating this sensing system to a model-based control/optimization solution will enable increased robustness, flexibility and optimized performance by reducing conservatism in operation while maintaining all operability constraints. In Phase III of the program, under Task 3, the developed sensing system solution utilizing a combination of available online sensors and "virtual" sensing through online model-based estimation using EKF, was coupled with the developed MPC solution. 


\section{Task 3 - Control System Design}

The objective of this task was to develop an advanced controls solution that enhances the level of automation and optimizes the operation of the gasification section in an IGCC plant, for both steady state and transient operation in startup as well as nominal power generation operation modes. Current operation/controls of the gasification section involves a combination of (i) lower-level simple single-input single-output (SISO) controls for variables like level, pressure, flow and temperature in key process units where they are measured online, and (ii) higher-level operator supervision and operation based on experience and simple guidelines. There is an opportunity to improve the overall gasification section operation performance through more advanced controls. Motivated by this, a model predictive controller (MPC) was designed to achieve the higher-level controls/operation objectives for the overall gasification section. MPC is a model-based controls solution that couples real-time transient model simulations and predictions with online real-time dynamic system optimization subject to all operability, safety or hardware constraints. This combination of dynamic model-based predictions and online constrained optimization provides a significant value in using MPC for improved steady state and transient operation at a section or plant level, with the flexibility to optimize for any desired objective that can be updated as the operation mode varies. It should be mentioned that MPC is used as an "optimizing supervisory control" at the section and/or the plant level, integrated with the lower-level SISO controls for each process unit, i.e., it does not replace the lower level controls. This also is practically valuable since an MPC solution can be implemented as an addition to existing lower-level controls in an operating plant without having to take the risk of completely replacing the existing controls.

For this task, initially in Phase I of the program, high-level controls requirements were identified for the gasification section for the key transient operations - startup (pre-heating and post-ignition pressure ramp up), turndown and fuel changes. The controls requirements were identified in terms of key performance objective (output tracking and minimization/maximization of some performance metric), sources of variations or disturbances, available control input variables for performance optimization and magnitude and rate constraints on all control inputs and magnitude and rate constraints on process outputs for operability. There are three distinct phases during startup in the gasification section: (i) pre-heating, (ii) ignition, and (iii) ramp up and pressurization from ignition to high-pressure steady-state operation. In particular, the pre-heating phase takes a long time, wherein the gasifier refractory and the RSC are heated following a prescribed temperature profile, which is designed to be conservative to avoid critical thermal stress limits. A model-based control strategy where the critical stresses are monitored online and the transient heating profile is optimized to maintain these stresses below acceptable limits is expected to provide significant reduction in the total pre-heat time. MPC simulation studies with pre-heating of gasifier and RSC indeed indicated significant reduction in preheat time, while maintaining all thermal and stress constraints. On the other hand for nominal operation (i.e. after startup and at design pressure conditions), a key control input is the oxygen-to-carbon ratio, which is decided based on prior experience and operator knowledge. In the absence of online real-time feedback for key constraint and performance variables (e.g. carbon conversion, efficiency, slag viscosity), this is often set for a conservative operation to stay away from operation boundaries. A model-based control strategy that allows continuously monitoring the constraint and performance 
variables, and also coordinates other control inputs along with the oxygen-to-carbon ratio, is expected to yield improved steady-state performance (e.g. minimize oxygen consumption, or maximize overall efficiency) as well as transient operation (e.g. achieve faster throughput/load changes) with coal as well as coal-petcoke blended fuels.

In Phase II of the program, in parallel with the sensing system design in Task 2, initially a full "state feedback" MPC was designed, i.e., assuming ideal or perfect sensors for all needed state and output variables. The state feedback MPC was implemented on the gasification section model and systematic transient simulations were performed to evaluate the process operation entitlement, i.e., the best performance improvements achievable at steady state as well as during transients subject to all operation constraints for startup and nominal operation modes. In Phase III of the program, the state feedback MPC was subsequently coupled with the sensing system from Task 2 to obtain the overall integrated sensing and control system. Figure 54 shows a schematic representation of the overall sensing and control system. In particular, at any time sample, the measurement from the online sensors is used to update the state (and parameter) estimates for the model using the EKF described in the previous section. Then the updated dynamic model is used to predict the transient response of the process over a desired future prediction horizon for a specific control input sequence, which is coupled with online constrained optimization to achieve the optimal solution enforcing all constraints and achieving desired tracking or optimization performance. The first step of the calculated optimal control sequence is implemented and the whole sequence is repeated at the next time step. Owing to repeated online model-based prediction over a long future horizon, it is imperative that the model used for prediction can be simulated much faster than real-time.

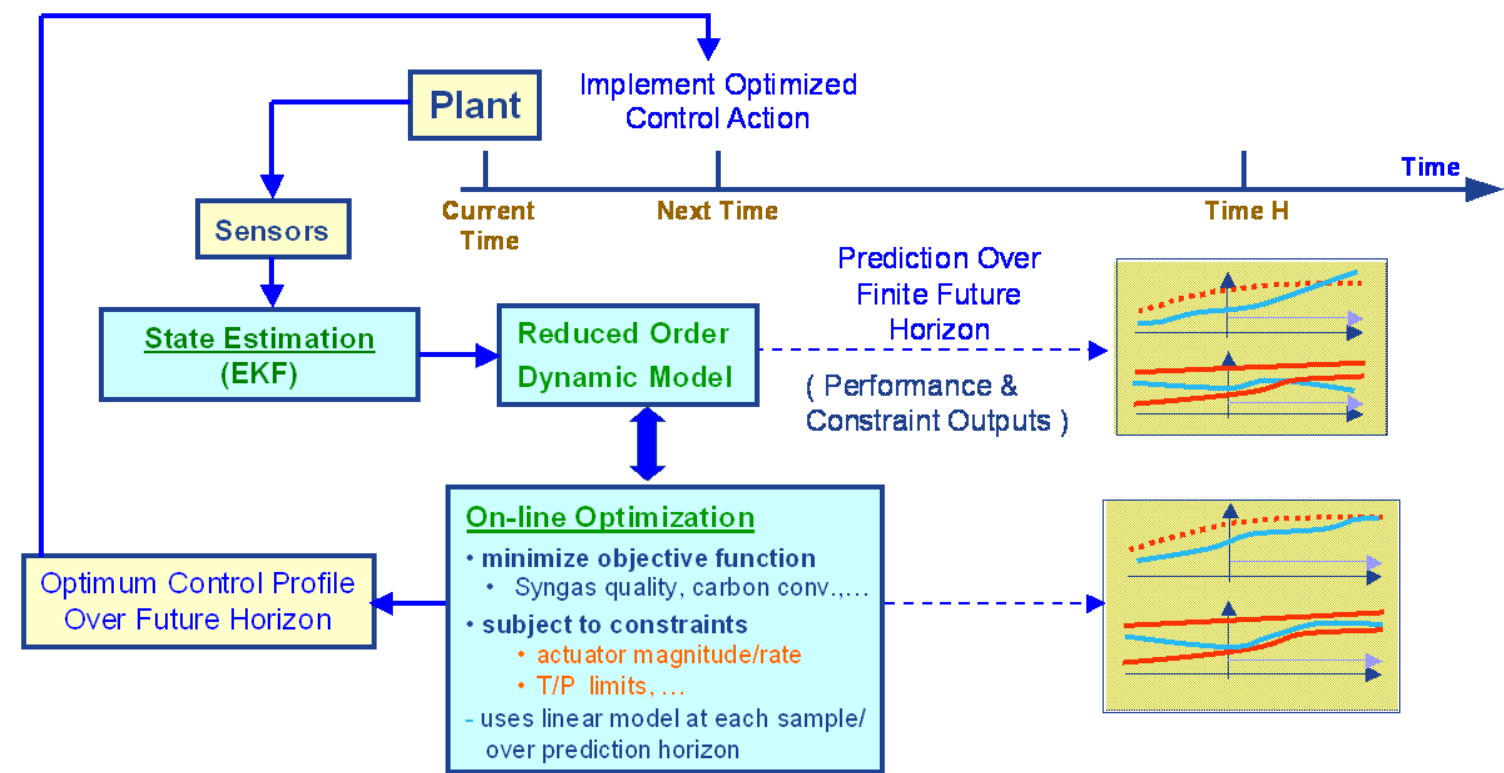

Figure 54: Schematic representation of the Integrated Sensing \& Control Solution using EKF \& MPC. 


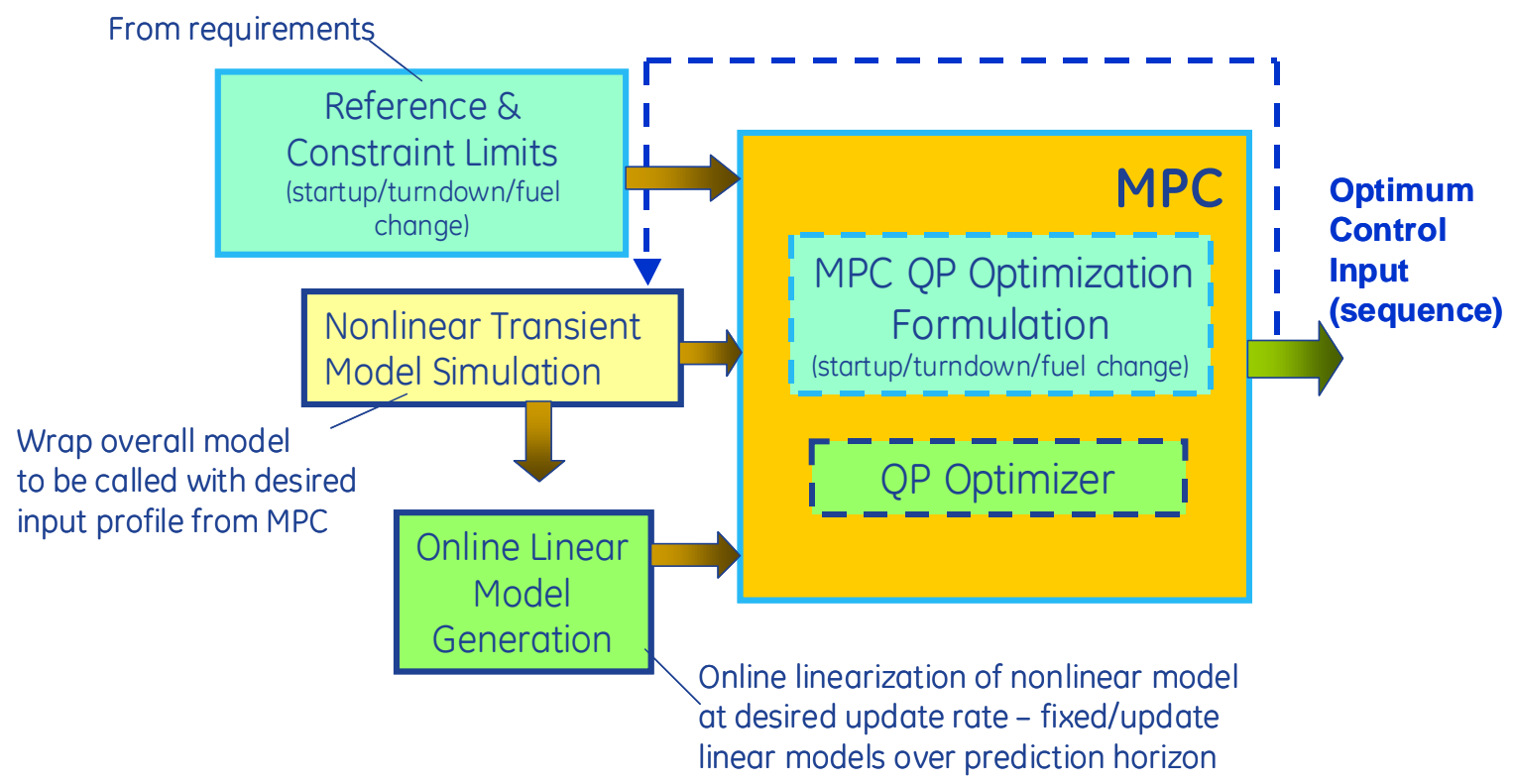

Figure 55: MPC implementation.

After completing the gasification section model in Matlab/Simulink ${ }^{\circledR}$ from Task 1, a model predictive controller (MPC) was implemented in Simulink to optimize the gasification section operation at steady state and during transients. Figure 55 shows the MPC implementation framework in Simulink, with different components including (i) a target reference and constraint generation block based on the control requirements - it provides the output reference trajectories to be tracked, performance outputs to be minimized and the constraint limits on all control inputs and process outputs, (ii) a prediction block to simulate the nonlinear dynamic model over a desired prediction horizon with a specified control input sequence, (iii) a linear model generation block to linearize the model online at desired intervals for specified state and input conditions, and (iv) a quadratic programming (QP) formulation and solution block to calculate the optimized control action over the future horizon.

The MPC implementation was tested through comprehensive simulation studies for startup pre-heating of gasifier and RSC as well as for nominal operation at steady state or turndown with nominal coal and coal-petcoke blended fuels. The simulation results are summarized in the next section.

\subsection{MPC for Startup Pre-heating Optimization}

One of the key transients to be improved during plant startup is for the gasifier refractory pre-heating. Currently, a prescribed temperature profile is used during pre-heating in an "open-loop" strategy, and it takes a significant amount of time to complete the pre-heating. In order to optimize the startup pre-heating profile subject to constraints on the thermal stresses in the refractory bricks, a model predictive controller (MPC) was developed based on the refractory thermal and stress models, process constraints and control targets. Compared to the current open loop schedule where the heating process follows a predetermined hot surface temperature profile, MPC can achieve the preheating requirements in a minimum time span while obeying all the constraints and process 
requirements. The optimized pre-heating from MPC allows saving several hours in the gasifier preheating process.

In late 2008, the dynamic thermal model of gasifier refractory layers (in Matlab/Simulink® platform) and the mechanical model of stress responses with a temperature distribution across the layers (using ANSYS® software package) were built and checked with some results from other design tools. These models were first used to calculate the open loop thermal and stress responses for the current "open-loop" pre-heating strategy. In order to have a consistent comparison between the open-loop strategy and the MPC optimized operation, the maximum values of the tensile and compressive stresses observed during open loop operation were used as the constraint limits for MPC optimization. In other words, the optimized MPC pre-heating strategy was forced to obey the same maximum tensile and compressive stresses as obtained following the current (open-loop) preheating strategy.

As in the open loop operation, the MPC control uses the inner surface temperature of the gasifier as the manipulated variable to preheat the refractory via heat conduction through the layers of refractory. The goal of the process is to raise the overall temperature level of the refractory from startup ambient conditions to a desired final value; therefore MPC uses a weighted average temperature of the innermost refractory layer as the control target, which should be raised to the final value following a reference trajectory. The reference trajectory is set to be significantly faster than the baseline so that while tracking this reference, operability constraints become active and are the limiting factor for fast preheating. The constraints come from two aspects: the maximum heating capacity of the burner as the process input (manipulated variable), and the tensile/compressive stress limits of the refractory material developed during the heating process (output variables). The heating capacity limit used in MPC was implemented as the maximum ramp rate achievable for the inner surface temperature used in the open loop operation - same as the max ramp rate used in the baseline open-loop strategy for consistency. The stress constraints are the tensile and compressive stress limits, which were determined from the maximum stress levels in the open loop operation. Thus, the MPC optimization is performed to minimize the startup pre-heating time with the same operability constraints as observed in the baseline open-loop pre-heating schedule.

MPC optimization was studied through transient simulation of the gasifier pre-heating. Clearly, the critical constraints for pre-heating are the tensile and compressive stresses in the refractory bricks. The observed stresses are a function of the gasifier geometry. In particular, there are two distinct design options wherein (i) the design geometry is such that during the pre-heating, the gaps between the refractory bricks close due to thermal expansion, and thus lead to significant compressive hoop stress buildup, or (ii) the design geometry is such that the gaps between the refractory bricks do not close up even at the target pre-heating temperature, and thus compressive stresses in the refractory bricks remain low - rather the tensile stresses become the limiting constraint. MPC optimization studies were performed for both design cases.

The simulation results in Figure 56 show the comparison of the MPC optimized performance (in green) against the baseline open-loop operation (in red) for the case when the gaps between the refractory bricks are designed to close down. The top figure 
shows that the MPC optimized pre-heating is completed approximately $22 \%$ faster than the baseline strategy. Also, note that the optimized pre-heating hot surface temperature profile has a very different transient compared to the open-loop one. The open-loop strategy starts with a slow temperature rise initially and gradually increases the rate of temperature increase. In contrast, the MPC operation starts with the maximum rate of temperature increase initially and then slows down during intermediate periods when the compressive or tensile stresses are active. The bottom figure shows a comparison of the tensile (positive) and compressive (negative) stresses compared to the max limits (obtained from the peak values in open-loop strategy at $41 \%$ for tensile and $72 \%$ for compressive stresses). Note that the faster pre-heating implies an earlier time when the tensile stress rises to the max limit and then rides this limit for a while causing the preheating temperature profile to slow down. Thereafter, the gaps between the refractory bricks close down, causing sharp buildup in compressive hoop stress (and correspondingly a reduction in tensile stresses) to the max compressive stress limit. Ultimately, as the pre-heating temperature is achieved, the thermal gradients reduce and the tensile and compressive stresses also reduce towards the end of the simulation run.

Figure 57 shows a similar comparison of the open-loop operation (red) and MPC optimized (green) operation for the design case when the gaps between the refractory bricks do not close and thus tensile stresses build up monotonically to the end of the preheating to a slightly higher value, while the compressive stresses are far from constraint boundaries. Running to the same tensile stress limits as in the open-loop operation (slightly higher limit at 53\% compared to previous design case), the MPC optimized operation achieves approximately $25 \%$ faster pre-heating. The MPC optimized operation in this case is simpler, following the maximum heating rate until eventually the tensile stress is active at the max limit, causing a slight slowdown of the heating rate. 

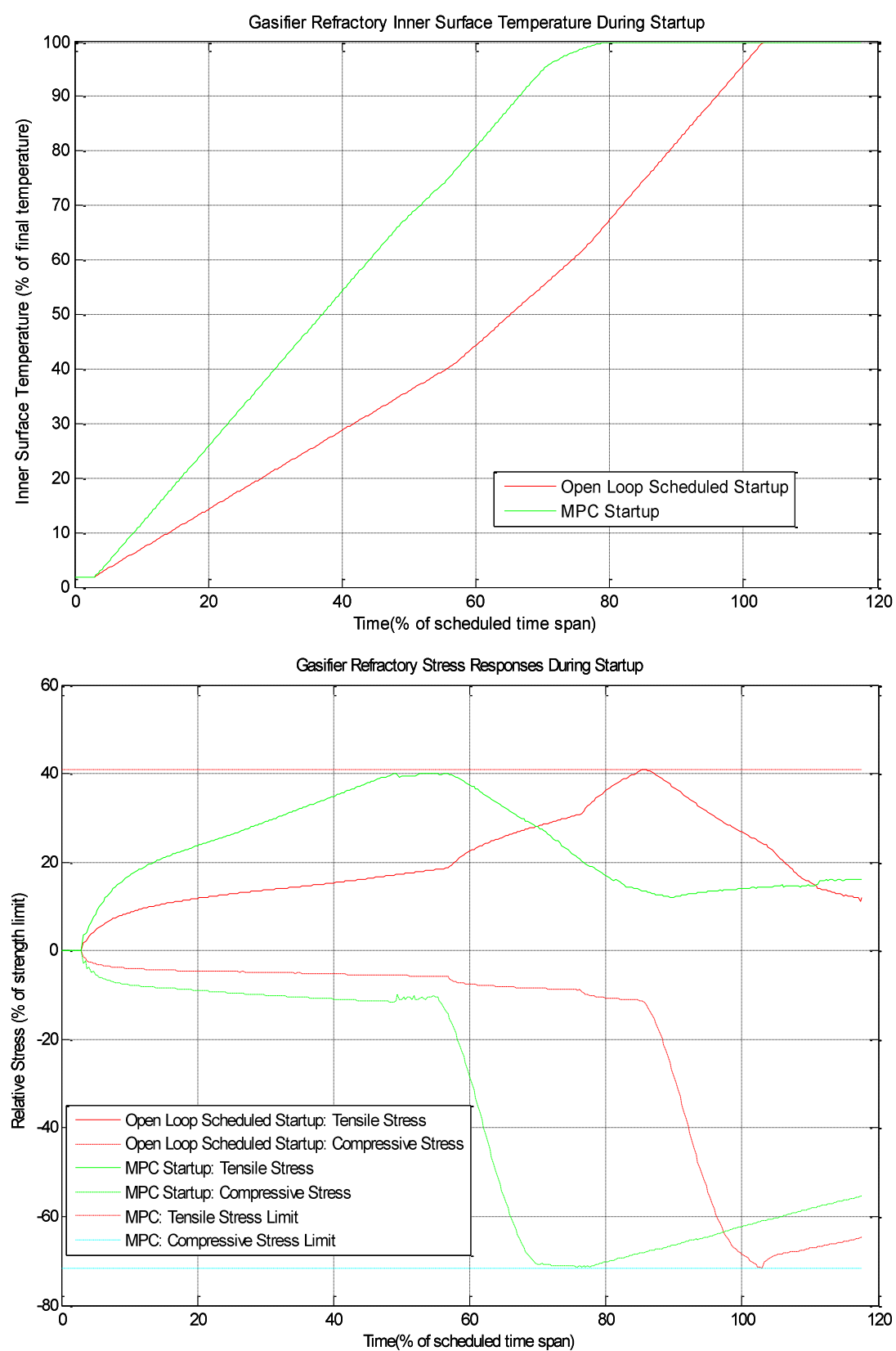

Figure 56: Gasifier startup preheating and thermal stresses - Open-loop schedule and MPC optimized profile. Design case with contact between refractory bricks. 

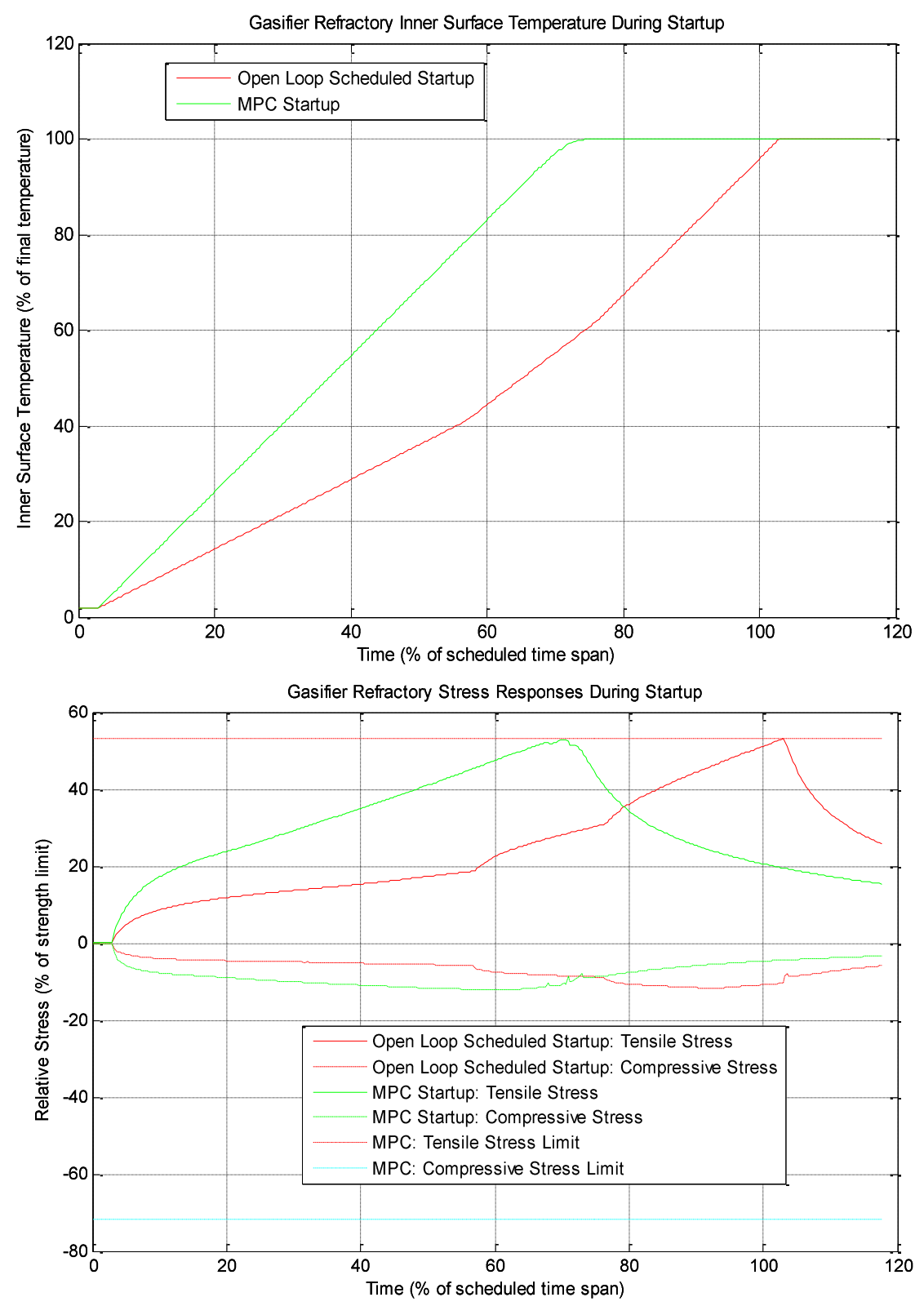

Figure 57: Gasifier startup preheating and thermal stresses - Open-loop schedule and MPC optimized profile. Design case with no contact between refractory bricks.

While the above simulation runs show significant improvement in startup preheating time for the gasifier refractory using MPC, another set of simulations were also performed to simultaneously reduce startup preheating time as well as the maximum tensile and compressive stresses on the refractory bricks. These simulations showed that both could be achieved at the same time with a trade-off between the two depending on the design/operation needs.

Similar to the gasifier pre-heating, MPC optimization was studied for the RSC pre-heating as well. For the RSC, medium pressure steam is fed to the HP drum and the resulting hot water is circulated through the RSC tubes to pre-heat them gradually to the desired 
temperature. The pre-heating leads to transient temperature differences between the tubes that heat faster and the outer shell that heats slower, causing an increase in thermal stresses. The current operation limits the temperature difference between the tube and the shell to a desired value as an indirect means to limiting the thermal stresses. Figure 58 shows the MPC optimized pre-heating of the RSC. The plot on the top left shows the target tube temperature reference (red dashed), which is set to be aggressively fast. The actual tube temperature profile is shown in blue, which reaches the desired final value in approximately ten hours. The other figures in the top half show the optimized profiles for the drum pressure (rising gradually due to steam injection) and the steam flow rate (negative values imply feed into the drum as opposed to positive values during normal operation when steam is withdrawn from the drum). The figures in the bottom half show the difference between the tube and shell temperatures (blue), running against the maximum allowed limit (red dashed) - the last figure shows the comparison of the tube and shell temperatures, with the shell temperature lagging behind the tube temperature. Also, the stresses in the critical component in the RSC are shown in blue compared to the max strength limits in red. For the allowed temperature difference between the tube and shell, there is still a significant margin available for the stresses, indicating available opportunity to speed up the pre-heating more if running to the stress limits instead of the temperature difference limit.
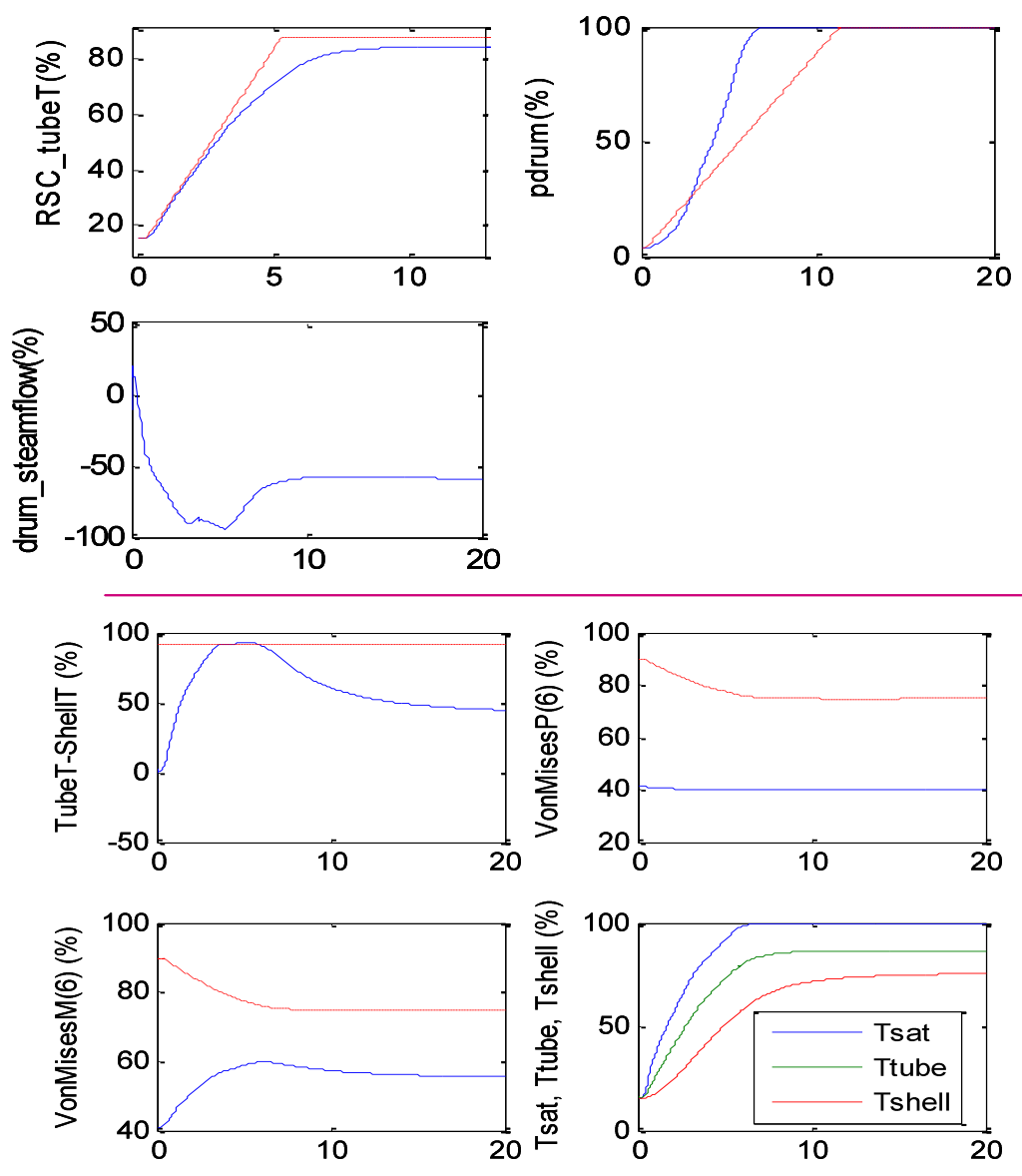

Figure 58: MPC optimized pre-heating of RSC. 


\subsection{MPC for Nominal Operation Optimization with Ideal Sensors}

This section summarizes the results of simulation studies with MPC using ideal sensors (i.e., state feedback). The simulations were performed with a mix of bituminous coals, as well as with a 50-50 blend of bituminous coal and petcoke, and show the improvement in both steady state and transient operation.

\subsubsection{MPC for Nominal Operation Optimization with Coal}

This section presents results on MPC optimized operation for nominal operation with coal fuel at part-load (50\%) and baseload steady state conditions as well as during load changes (turndown) between baseload and part-load conditions. More specifically, at steady-state conditions, the gasification section operation is optimized to minimize the oxygen consumption subject to operability constraints, which in turn reduces the internal electricity consumption in the air separation unit (ASU). On the other hand, MPC is used to optimize the load transients (turndown) between partload and baseload to allow faster turndown subject to limiting constraints. The varying optimization objectives within the MPC framework during steady state and transient operation allow a flexible and optimized overall plant operation to achieve desired objectives based on operation mode. For instance, at steady-state conditions MPC can be used to optimize for minimum oxygen consumption, maximum carbon conversion, maximum efficiency or maximum net electrical power output as desired. On the other hand, during load transients, MPC can be used to maximize the ramp rates to get to desired final power output in the least time.

MPC can be used at the gasification section level to optimize the operation through coordinated use of multiple control inputs including coal slurry feed, recycle $\mathrm{CO}_{2}$ feed, oxygen feed, as well as setpoints for lower level controllers like HP steam drum and syngas pressure. While optimizing the performance by MPC, it is still important to track key plant outputs (e.g. net electricity output, syngas pressure) at desired values. The gasification section operation allows a range of operation conditions with varying combination of inputs like oxygen feed, slurry feed, recycle $\mathrm{CO}_{2}$ feed, HP drum pressure etc., that yield the same desired net electricity output, thereby allowing degrees of freedom in operation that can be exploited to optimize for additional performance objectives (e.g. minimize oxygen consumption).

\begin{tabular}{|l|c|c|c|c|}
\cline { 2 - 5 } \multicolumn{1}{c|}{} & \multicolumn{2}{c|}{$\begin{array}{c}\text { Steady State Operation } \\
\mathrm{O}_{2} \text { saving }\end{array}$} & \multicolumn{2}{c|}{$\begin{array}{c}\text { Transient Operation } \\
\text { Load Rate }\end{array}$} \\
\hline \multicolumn{1}{c|}{ Manipulated Inputs } & $50 \%$ Load & Full Load & $\begin{array}{c}\text { Ramp Up } \\
50 \%->100 \%\end{array}$ & $\begin{array}{c}\text { Ramp Down } \\
100 \%->50 \%\end{array}$ \\
\hline $\begin{array}{l}\text { Baseline (Fixed Recycle } \mathrm{CO}_{2}{ }^{\prime} \\
\text { Water, RSC Drum Pressure) }\end{array}$ & - & - & $X$ minutes & Y minutes \\
\hline Recycle $\mathrm{CO}_{2}$ & $7.9 \%$ & $4.89 \%$ & $0.9 \times$ minutes & $0.8 Y$ minutes \\
\hline $\begin{array}{l}\text { Recycle } \mathrm{CO}_{2}+\text { RSC Drum } \\
\text { Pressure }\end{array}$ & $8.0 \%$ & $4.93 \%$ & $0.8 X$ minutes & $0.75 Y$ minutes \\
\hline $\begin{array}{l}\text { Recycle } \mathrm{CO}_{2}+\text { RSC Drum } \\
\text { Pressure + Slurry Water }\end{array}$ & $8.0 \%$ & $5.0 \%$ & $0.8 X$ minutes & $0.75 Y$ minutes \\
\hline
\end{tabular}

Figure 59: Summary of steady state and transient optimization with MPC using different control inputs 
In the initial MPC simulation studies, various control inputs including the slurry feed rate and oxygen feed rate, recycle $\mathrm{CO}_{2}$ flow rate, $\mathrm{HP}$ steam drum pressure setpoint, and the water content in the slurry feed were used to explore the optimization of the gasification section at steady state and during load transients. In the existing operation of the gasification section, the water content in the slurry is maintained at a fixed value owing to limitations on slurry flowability. While it is possible to vary the water content slowly and to a small extent, it is not desirable. The use of these control inputs for potential improvement in operation was explored to identify the relative advantage of each input. More specifically, the gasification section operation was optimized at partload and baseload conditions for minimized oxygen consumption, and during load transients for faster ramp rates. The results are summarized in Figure 59. Clearly, slurry throughput and oxygen flow rate have to be used as control inputs, to track desired net electric power output at different load conditions. A key question was to identify the benefit of using the additional inputs like recycle $\mathrm{CO}_{2}$, HP steam drum pressure and slurry water content in terms of improving steady-state and transient performance. From the results, it is clear that recycle $\mathrm{CO}_{2}$ and $\mathrm{HP}$ steam drum pressure are the primary control inputs, allowing up to $8 \%$ reduction in oxygen usage at steady state, and $20-25 \%$ improvement in ramp rates during transient load changes (turndown). The additional advantage of using the slurry water content was minimal, so it can be discarded from the control inputs in MPC.

Figure 60 shows the MPC simulation results at partload (50\%) steady-state operation, where it is configured to minimize the oxygen consumption. A key challenge in the MPC formulation was that optimization for performance objectives would trade-off against the tracking performance. This is not desirable, since tracking objectives (e.g. net electricity output) have a high priority and performance optimization should not be done at the expense of tracking performance. To address this, the MPC formulation was updated to distinguish between tracking and performance optimization objectives, and only allow performance optimization subject to meeting the tracking objectives. The plots on the top show the setpoint (red-dashed lines) for the tracking outputs, i.e. net electricity output, and syngas pressure), and the actual outputs (blue) which indeed track the control setpoint. The plots below show the variation in syngas and steam flow rates as MPC optimizes the operation for minimized oxygen consumption while still tracking the control outputs. The plots on the right show the variation of various control knobs (blue) compared to the maximum (red) and minimum (light blue) limits. The variable G_Totalflow_sf denotes the variation in slurry feed rate, while G_slurrywater_sf denotes the variation in slurry water content (held constant at baseline value), G_CO2_sf denotes variation in recycle $\mathrm{CO}_{2}$ flow rate, and G_PetCk_sf denotes the change in petcoke fraction in the fuel (held constant at zero for coal operations). Note that the oxygen use is reduced by roughly $8 \%$ and this minimum is achieved at minimum recycle $\mathrm{CO}_{2}$ feed flow rate. The plots at the bottom show additional important process variables (e.g. carbon conversion, syngas LHV and $\mathrm{MWI}$ ) in blue compared to their minimum (light blue) and maximum limits (red). Clearly, MPC maintains these outputs within desired limits while achieving the minimum oxygen consumption. 

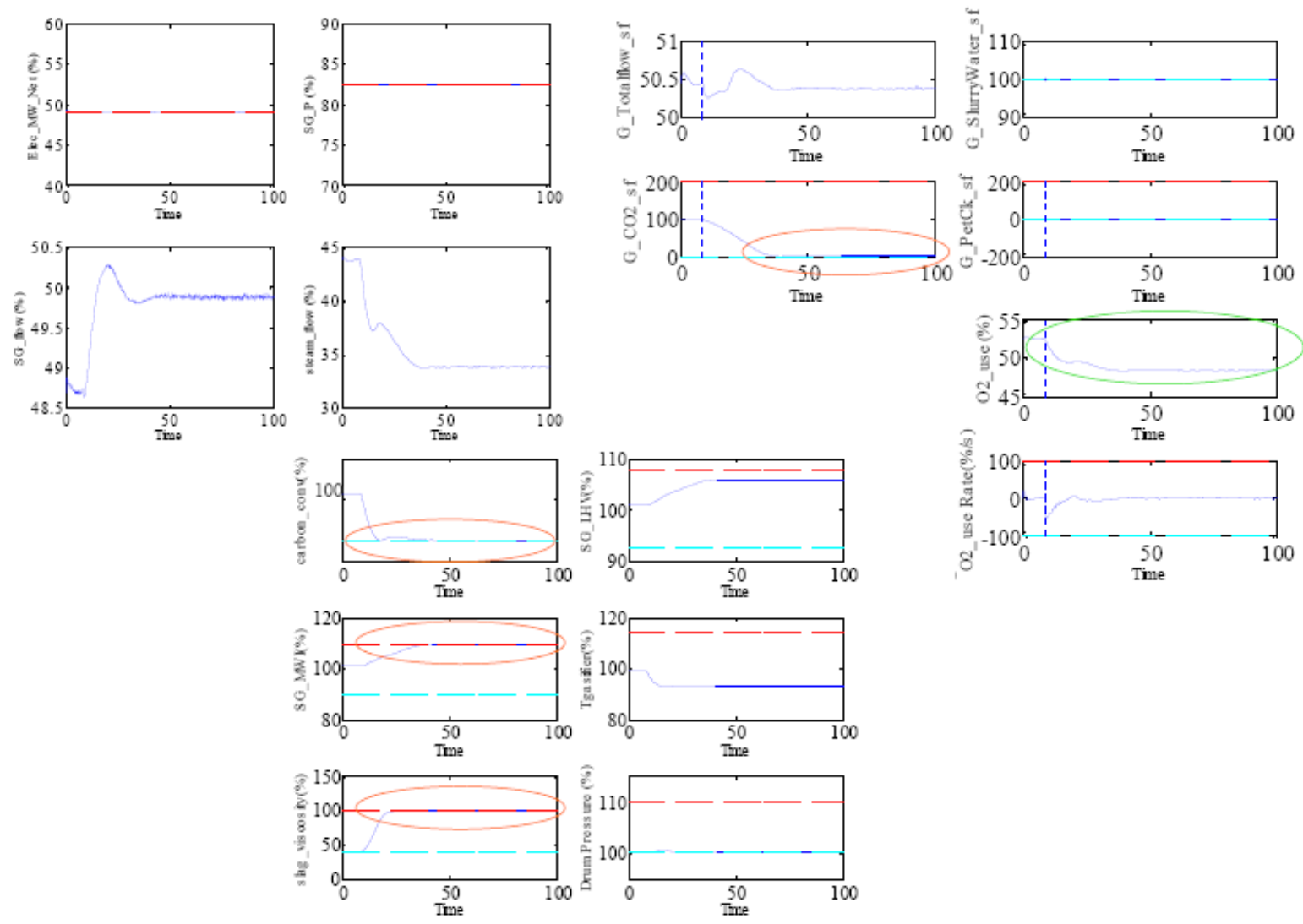

Figure 60: MPC performance for oxygen use minimization at $50 \%$ load operation with coal.

Figure 61 shows similar plots for transient operation optimization during a load ramp from partload $(50 \%)$ to baseload $(100 \%)$. In the baseline operation strategy, the load transients are limited by the oxygen supply rate of change from the ASU. In this simulation run, the ramp rate target (red) for the electric power output was set to be about $20 \%$ faster than the baseline rate. MPC is clearly able to follow this faster ramp rate. Note that during this load transient, MPC uses multiple control inputs like the slurry feed, oxygen flow rate, recycle $\mathrm{CO}_{2}$ flow rate and the HP drum pressure setpoint in a coordinated manner to enable following the faster ramp rate. This is a critical multivariable feature of MPC, which optimally coordinates multiple control inputs based on model predictions to achieve the desired control objective. Note that for this load transient, MPC increases the oxygen supply at the maximum allowed rate of change (limited by the ASU rate of change), while also transiently modifying the recycle $\mathrm{CO}_{2}$ and $\mathrm{HP}$ steam drum pressure. 

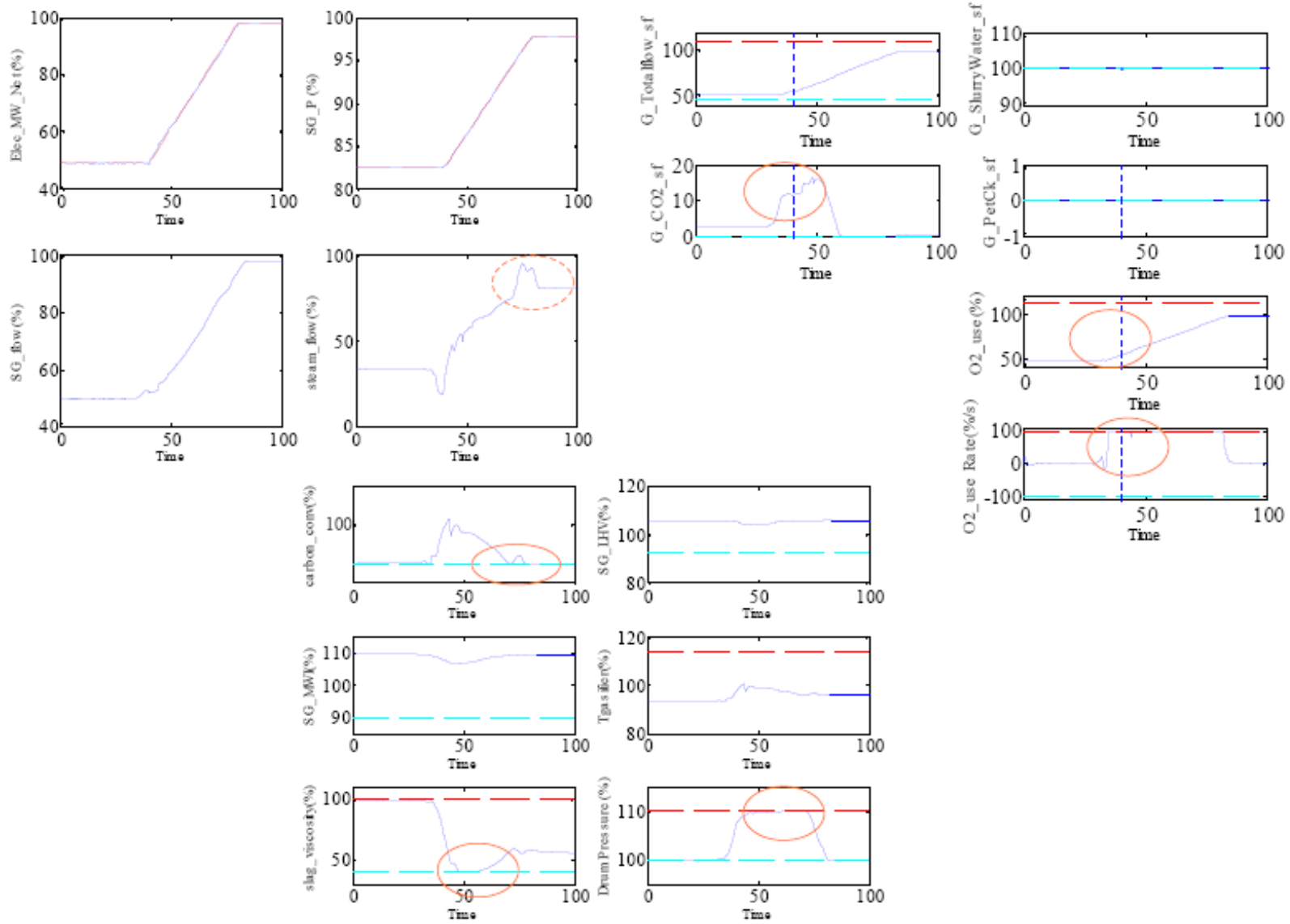

Figure 61: MPC performance for faster ramp up during load transients between $50 \%$ and $100 \%$ load for operation with coal.

Finally, Figure 62 shows an example long simulation run for about 10 hours showing different modes of operation and flexible optimization for each mode of operation. More specifically, initially, starting at partload $(50 \%)$ condition, MPC is used to minimize oxygen consumption, then maximize the ramp rate during the load change to baseload $(100 \%)$, followed by minimization of oxygen use at baseload steady-state, before following a fast turndown to partload and minimizing again for oxygen use at the final partload condition. Clearly, MPC uses the various control inputs in a coordinated manner to achieve each control/optimization objective during the various steady state and transient phases of operation, while maintaining all inputs and outputs within allowed limits. This illustrates the flexibility of using MPC for varying performance optimization depending on the needs for any operation mode. MPC was able to achieve $8 \%$ reduction in oxygen at partload, approximately $5 \%$ reduction in oxygen use at baseload and $20-25 \%$ faster ramp rates during the transients between partload and baseload, as summarized in Figure 59.

While oxygen minimization reduces the oxygen consumption at part-load, this has an adverse impact on the plant operation during ramp-up when raising the plant output from part-load to baseload. The ramp-up is limited by the capability of the ASU to ramp up its production of oxygen - starting from the minimum oxygen point makes this even more limiting a constraint during the ramp-up. This is addressed in the MPC formulation by allowing for a preparation phase for ramp-up. A key advantage of MPC is the ability to optimize for a future prediction horizon. This allows optimizing the operation of the plant in 
the preparation phase before the ramp-up is initiated. MPC optimizes the operation to increase the oxygen feed, while maintaining the constant net electricity output at part-load - this essentially provides a head start in the ASU ramp-up, a limiting factor, and thereby allows a faster ramp-up. Similar simulation runs were also done for a plant with fully fouled RSC, where MPC optimization modified the operation to address the slightly reduced steam generation due to the RSC fouling.
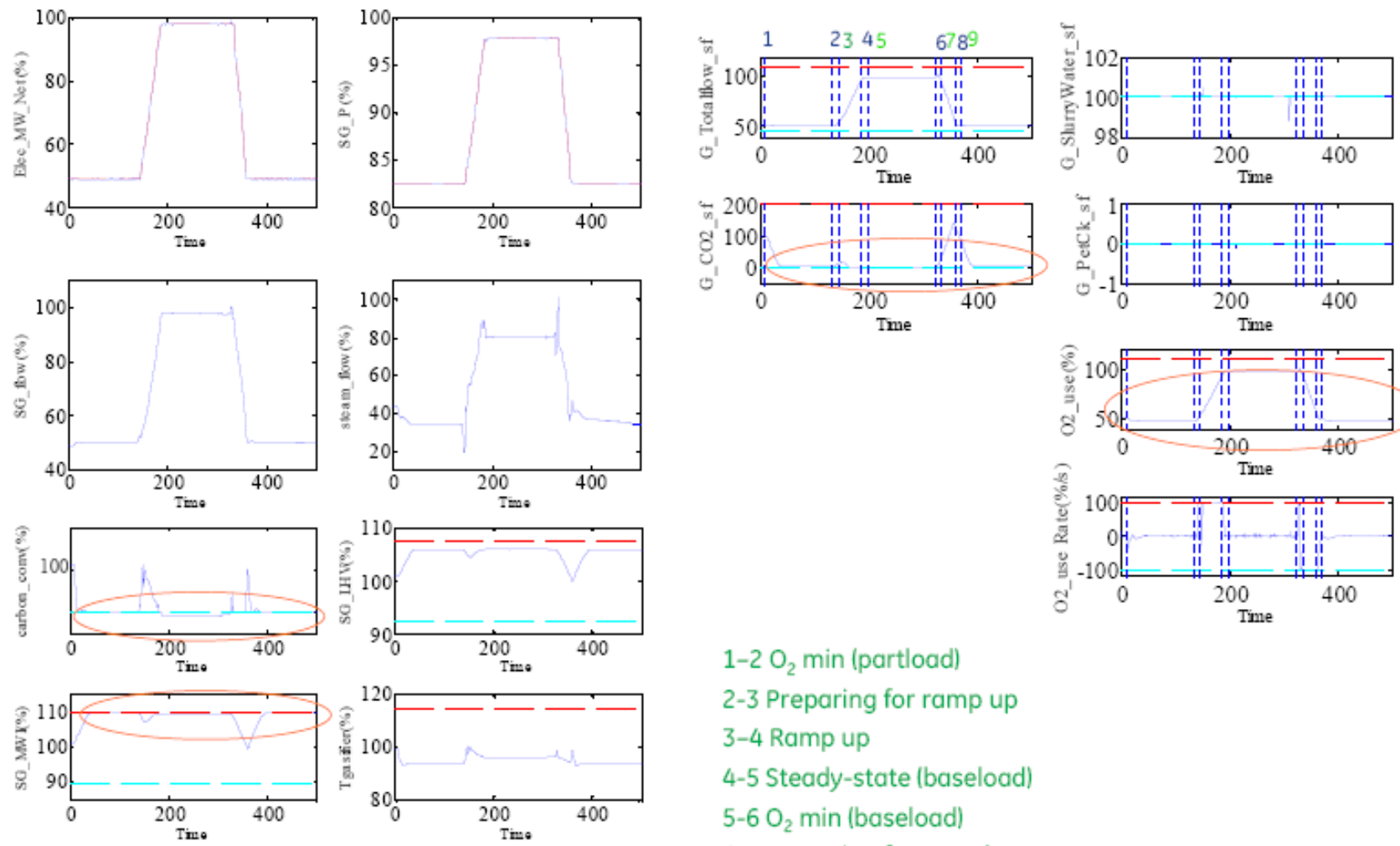

\section{$1-2 \mathrm{O}_{2} \min$ (partload) \\ 2-3 Preparing for ramp up \\ 3-4 Ramp up \\ 4-5 Steady-state (baseload) \\ 5-6 $\mathrm{O}_{2}$ min (baseload) \\ 6-7 Preparing for turn down \\ 7-8 Turn down \\ 9- $\mathrm{O}_{2} \min$ (partload)}
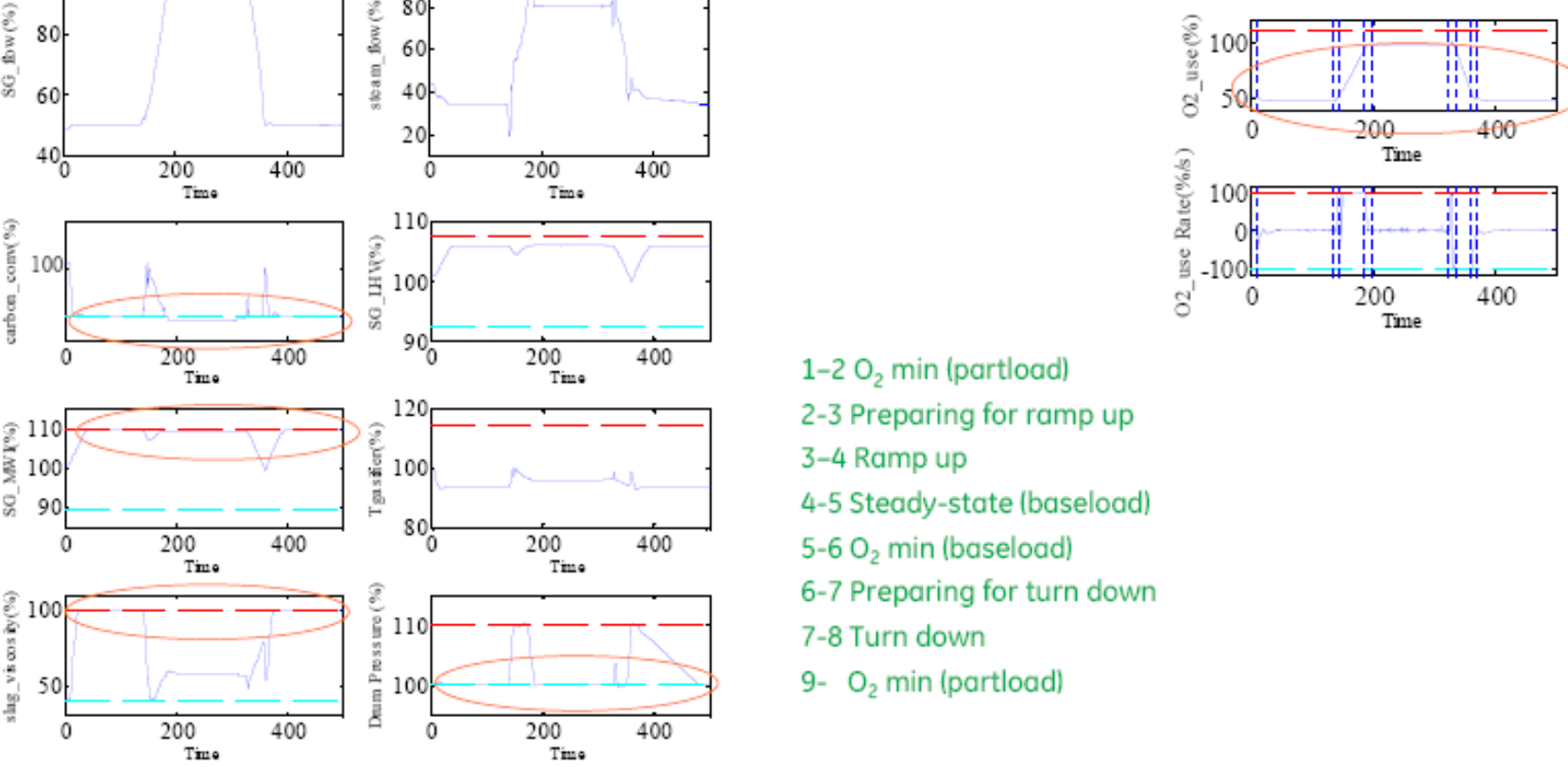

Figure 62: MPC optimization for nominal operation with coal during steady-state operation at part-load and baseload, and during turndown.

\subsubsection{MPC for Nominal Operation Optimization with Coal-Petcoke Blend}

This section presents results in MPC optimized operation for nominal operation using coal+petcoke blended fuel - considering blends with $0-50 \%$ petcoke. The MPC optimization was similar to coal-based operation discussed in the previous section, optimizing for minimum oxygen at steady-state conditions and faster ramp up/down during turndown. A key difference in operation with coal-petcoke blend compared to coal operation is the significant increase in the carbon content in petcoke with a corresponding increase of the fuel heating value (HV). Initially, simulation studies were performed at baseload and part-load comparing open-loop transition from coal to coal-petcoke blend, changing the petcoke from $0-50 \%$ following a ramp and keeping other operating parameters unchanged. In comparison, for the same ramp in petcoke fraction, MPC 
optimized operation maintained the required total net electricity output during the transition, while maintaining all operability constraints within desired limits.

Figure 63 shows a comparison of transient operation at part-load condition as the fuel is transitioned from $0 \%$ petcoke to $50 \%$ petcoke in (i) an open-loop manner (green), keeping all other operation parameters constant, or (ii) using MPC (blue) to maintain same power output and pressure conditions while maintaining all operability constraints within limits. For operation with petcoke-coal blends the slurry water content was allowed vary slightly by about $5 \%$. Owing to the fact that petcoke is significantly richer in carbon, under same operating conditions, the oxygen-to-carbon ratio drops and consequently the gasifier temperature also drops, leading to reduced carbon conversion and higher slag viscosity. On the other hand, despite the reduced carbon conversion, the syngas yield and quality (LHV, MWI) increase significantly, while the steam production reduces (due to reduced temperature). The overall net electricity production increases significantly. Due to the differences in the fuel characteristics, the operation of the plant has to be modified to maintain the same target electricity output, and maintain important operability constraints like slag viscosity, carbon conversion, syngas LHV and MWI within acceptable limits. The MPC operation is designed to achieve this as can be seen in the plots - it maintains the constant electricity power output by reducing the slurry feed and the oxygen feed while slightly increasing the slurry water content and recycle $\mathrm{CO}_{2}$. The transition to $50 \%$ petcoke blend operation leads to reduced temperature and thus, reaching the max limit on slag viscosity constraint. Note also that owing to the higher carbon content and fuel heating value, the syngas LHV is also high at the max limit.
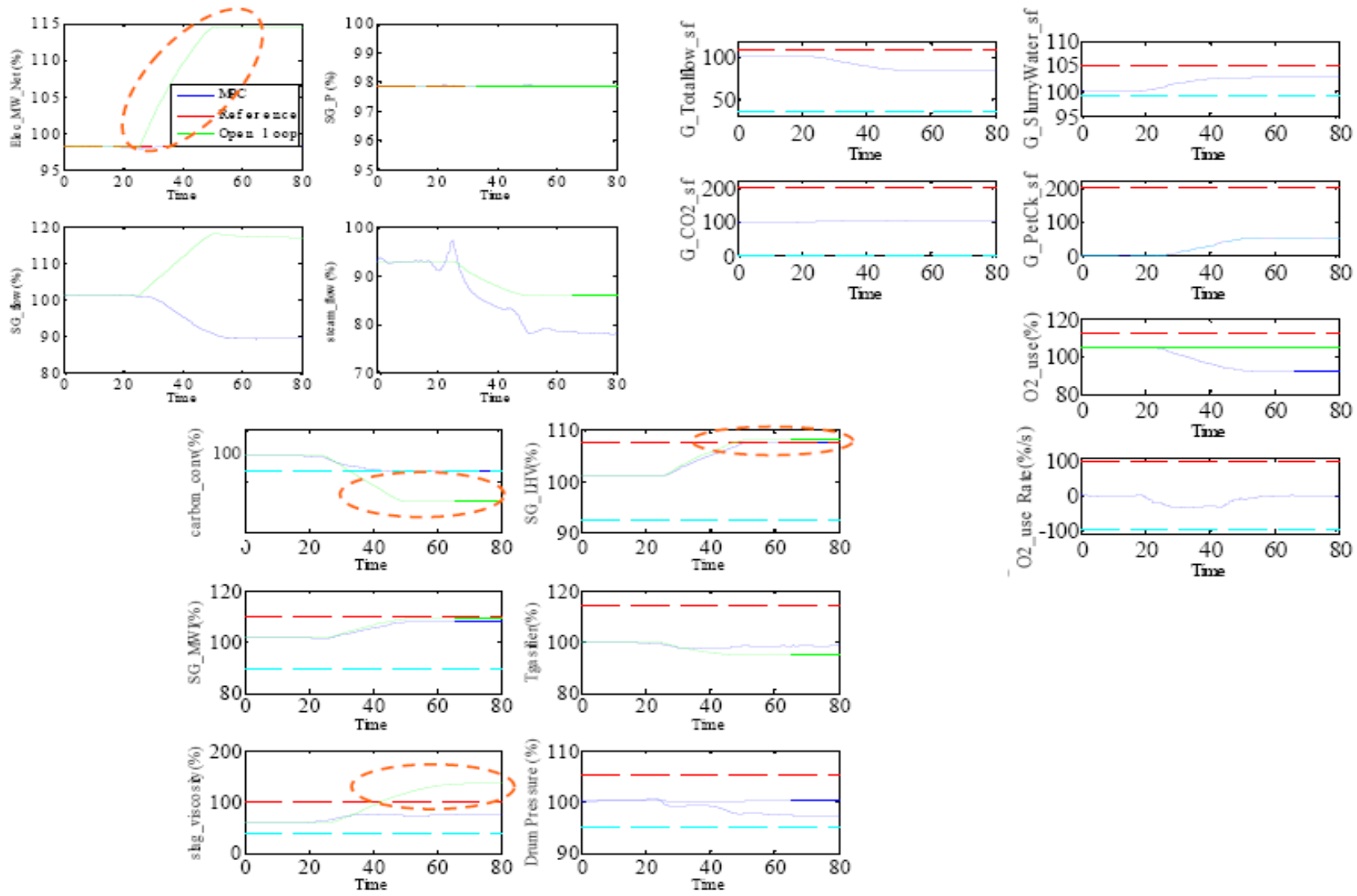

Figure 63: Comparison of open-loop operation (green) with change in petcoke fraction with MPC operation (blue). 
Similar to coal-based operation, MPC was configured to optimize for oxygen use at steady state operation while maintaining tracking outputs like net electrical power output and syngas pressure at nominal setpoint. In the absence of a reference baseline operation condition for comparison, this was studied by configuring MPC to operate at extreme conditions, i.e. minimize oxygen consumption and maximize oxygen consumption to identify the entitlement opportunity for oxygen use minimization. Figure 64 shows a comparison of the results for maximum oxygen use (green) and minimum oxygen use (blue), showing a potential to vary oxygen use by up to $5.8 \%$. Note that some of the critical operation constraints with petcoke include carbon conversion (due to the significantly higher carbon in petcoke) and syngas LHV. Also, unlike operation with coal, the optimum operation with petcoke is achieved with significant recycle $\mathrm{CO}_{2}$, again due to the high carbon content and carbon conversion constraint.
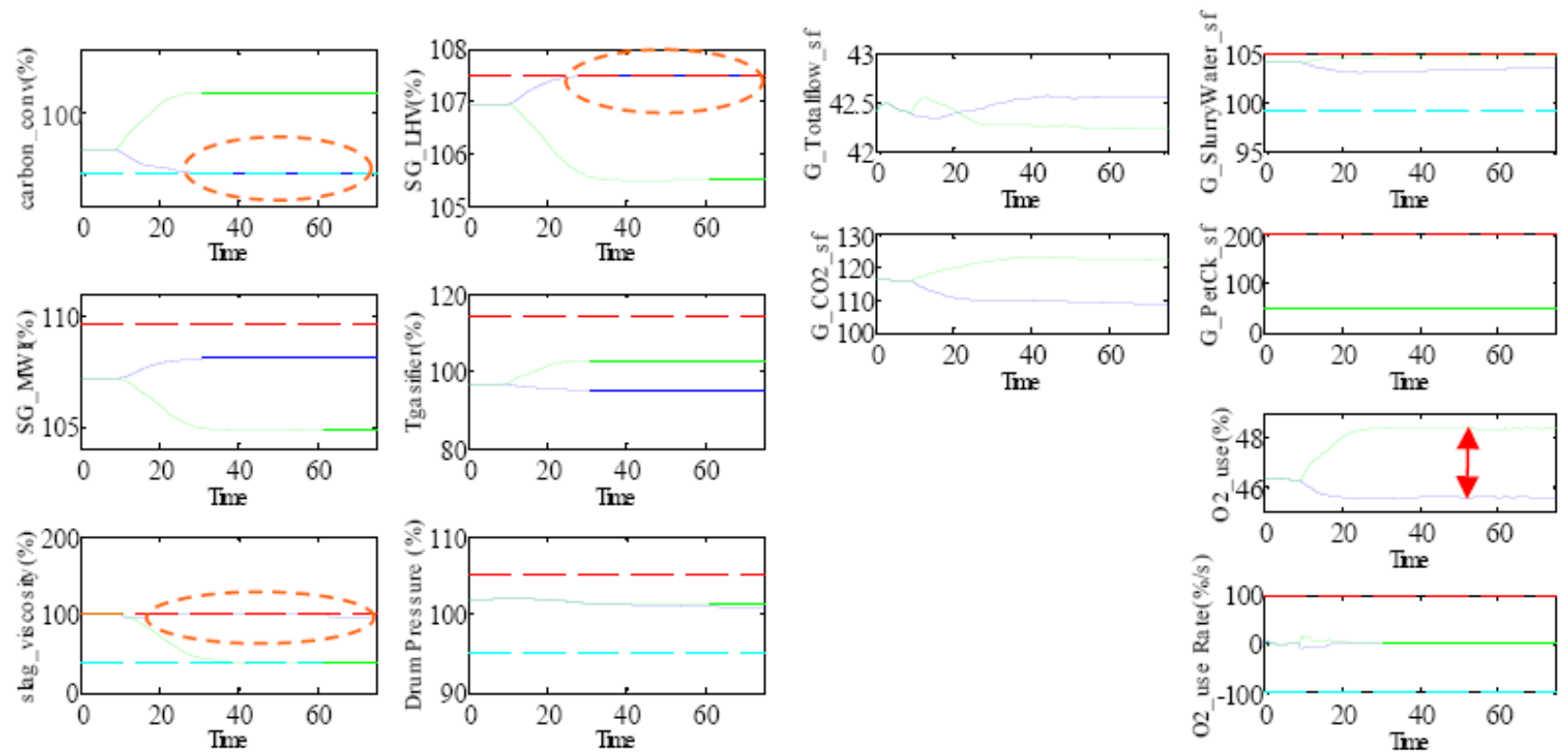

Figure 64: MPC optimized operation at part-load with 50\% coal-petcoke blend showing up to $5.8 \%$ variation in oxygen use between operation with maximum (green) and minimum (blue) oxygen consumption while maintaining the power output at $50 \%$.

Figure 65 shows the performance of the MPC optimized operation with $50 \%$ petcoke blend over a long simulation run showing multiple operation modes including steady-state conditions at part-load and baseload conditions as well as transient turndown between these conditions. Similar to the simulation studies with coal in the previous section, starting at part-load, (i) initially the operation is optimized to minimize the oxygen consumption, followed by (ii) optimization for ramp-up to base load, (iii) oxygen minimization at baseload, (iv) optimization for ramp-down to part-load, and finally (v) optimize again for oxygen minimization at part-load. As evident in Figure 64, the optimized operation with petcoke blend at partload runs against the min carbon conversion and max syngas LHV constraint limits - the higher fuel quality (richer in carbon) of petcoke blend leads to higher quality syngas. The constraint on the syngas LHV can be relaxed, in which case the optimized operation runs to the max syngas MWI limit instead. The opportunity for oxygen consumption reduction is lower for petcoke operation compared to coal operation due to the more constrained operation range with the higher carbon content. On the other hand, the turndown transients during ramp-up and ramp-down were able to 
successfully follow a $20-25 \%$ faster ramp rate compared to the ASU ramp rate that limits the rate of change of oxygen feed. Note that during the faster turndown transients, the oxygen feed, slurry water content, HP steam drum pressure and recycle $\mathrm{CO}_{2}$ feed are changed significantly in a coordinated manner to maintain the gasifier temperature needed for carbon conversion and slag viscosity constraints. Note also that unlike operation with coal, during operation with petcoke-coal blend, the recycle $\mathrm{CO}_{2}$ flow rate is never reduced to zero, again to achieve the high (and limiting) carbon conversion in the presence of significantly higher carbon content in petcoke.
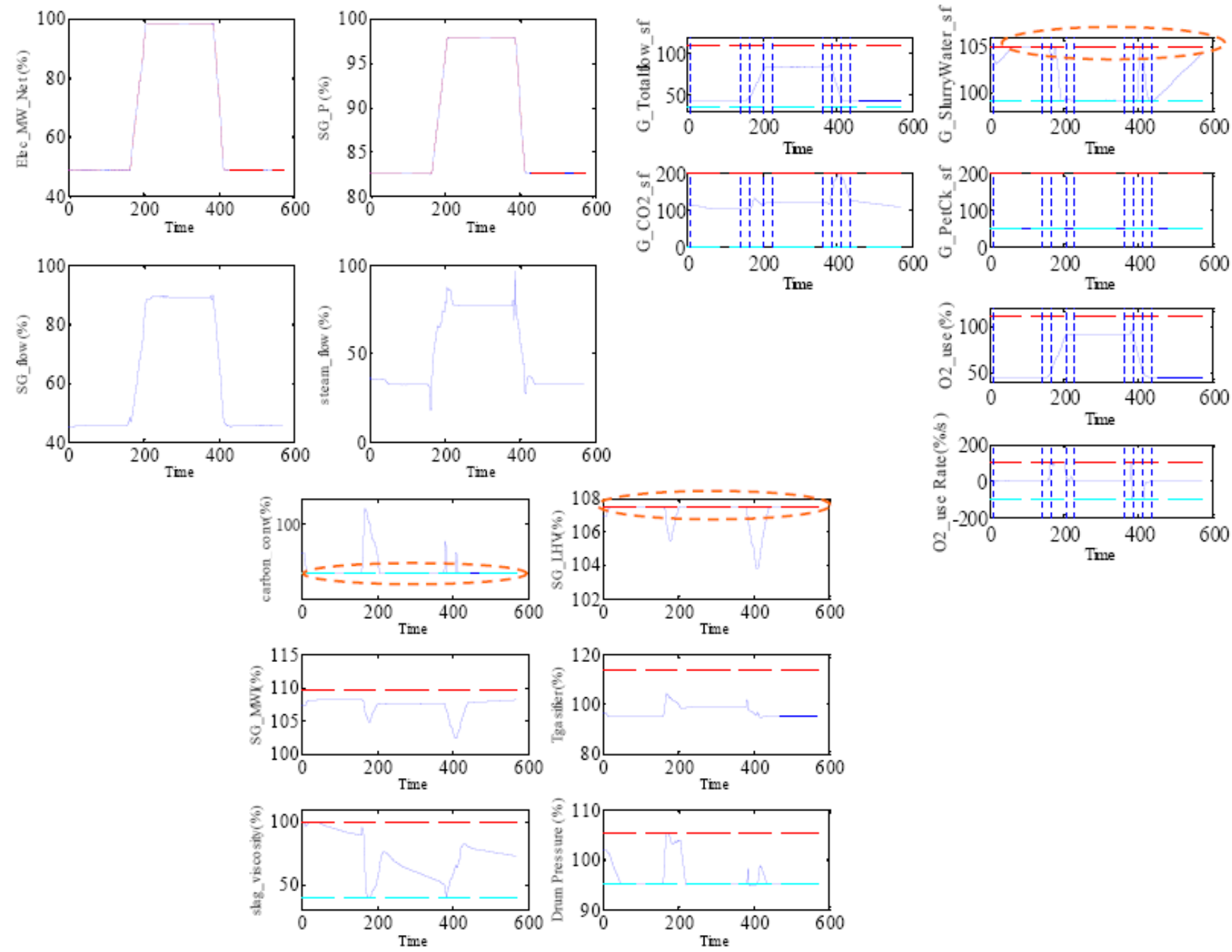

Figure 65: MPC optimized operation at 50\% part-load and 100\% baseload and during turndown with $\mathbf{5 0} \%$ petcoke-coal blend.

This section presented MPC simulation results using ideal sensors or state feedback, both for coal and coal-petcoke blends. The simulation runs show significant opportunities to optimize the plant operation at steady state as well as during transients. Also, MPC allows an increased flexibility in operation, with the ability to optimize for different optimization objectives (e.g. oxygen use minimization, carbon conversion maximization, efficiency or power output maximization at steady state, or faster transients during load changes) as desired during different operation modes. 


\subsection{Integrated MPC and EKF for Nominal Operation Optimization}

Figure 44 shows the architecture used in Phase II, wherein the MPC with ideal sensors, i.e. perfect knowledge of all state and output variables from the plant, and the EKF-based sensing system using a combination of online sensors and model-based estimation were developed and tested in parallel in an open-loop configuration. In Phase III, the separate designs for MPC with ideal sensors and the EKF-based sensing system were coupled to obtain the overall integrated sensing and control system, as shown in Figure 66.

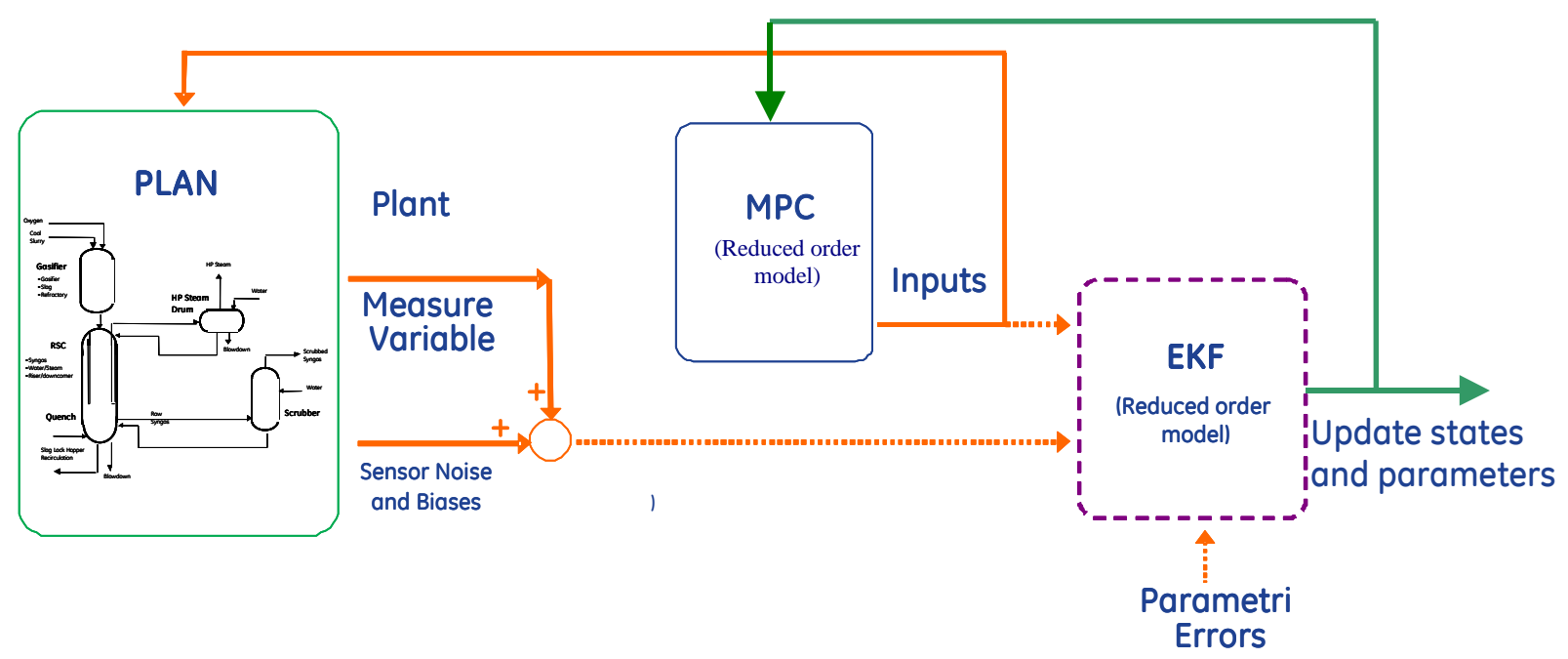

Figure 66: Schematic of Integrated EKF and MPC. The EKF uses sensor measurements to update the states and parameters for reduced order model used in MPC.

In a phased approach to this task, first the separate implementations of MPC and EKF were unified in a combined implementation, using the latest reduced-order model common to both and a common linear model generation routine to achieve high computational efficiency. Thereafter, the integrated control and sensing system was retuned for stability and performance robustness to random combinations of sensor noise, bias and modeling error. The final retuned system was studied using Monte-Carlo simulations with random sensor bias for both coal slurry feed and coal-petcoke blend. For all these simulations, the system is initially simulated in open loop with only the EKF enabled (without engaging MPC controller) for about 60 minutes. This initialization period is required for initial transients in estimated states and parameters in the EKF to settle down. Thereafter the MPC controller is engaged to study the performance of the integrated EKF-MPC system for steady state and transient operation. The simulation results for coal slurry feed based on a number of Monte-Carlo simulations with random combinations of sensor bias and modeling error (RSC fouling and gasifier kinetics) are presented next.

\subsubsection{Nominal Operation Optimization with Coal}

Simulations studies conducted with the integrated control and sensing system using coal slurry feed show good tracking performance during steady state as well as transient operations. Figure 67 and Figure 68 show one such result for a long simulation run with multiple steady state and transient operation phases. Figure 67 shows various phases of the simulation run. The simulation is initiated (point ' $A$ ') at base load condition with only the 
EKF enabled. The simulation runs for almost 60 minutes without any external control input (open loop). After that the MPC is enabled at point ' $B$ '. The MPC quickly tracks the net electrical output and scrubber syngas pressure to the respective reference set points. At a steady state point ' $C$ ' the MPC is configured for net electrical output maximization mode while respecting all the operational constraints (e.g., maximum slurry feed, ASU limit etc). Clearly, MPC is able to achieve about $2 \%$ higher net electricity output in this mode. At a later steady state operation point ' $D$ ' the net electrical output maximization mode is suspended in preparation for the net electrical output turndown. Operation phase ' $E$ ' to ' $F$ ' represents $25 \%$ faster ramp tracking in net MW as well as scrubber syngas pressure compared to the nominal operation to $50 \%$ part load. Since, it is normally not desirable for the IGCC plant to turn down all the way to zero load due to startup complexities, the end of this turndown transient may represent the parking of the plant during the low power demand period. During this period, one objective would be to minimize the oxygen consumption while maintaining certain minimum load (net electrical output as well as scrubber syngas pressure) - this in turn reduces the internal power consumption in the ASU and achieves higher plant operation efficiency. The point ' $G$ ' represents the starting point of such a phase. At point ' $G$ ' the MPC is engaged in the minimization of the oxygen consumption mode while maintaining $50 \%$ load. In this phase, MPC is able to reduce oxygen consumption by about $10 \%$. Phase 'I'-'J' of the simulation represents the ramp up transient tracking of net electrical output from $50 \%$ load to base load condition at $20 \%$ faster rate compared to the nominal rate. Once at the base load, the MPC is again engaged in the maximization of net electrical output mode at a steady state operation point ' $\mathrm{K}$ '. As mentioned, all through this simulation the scrubber pressure is also tracked to a given reference trajectory as shown in the bottom graph of Figure 67. 

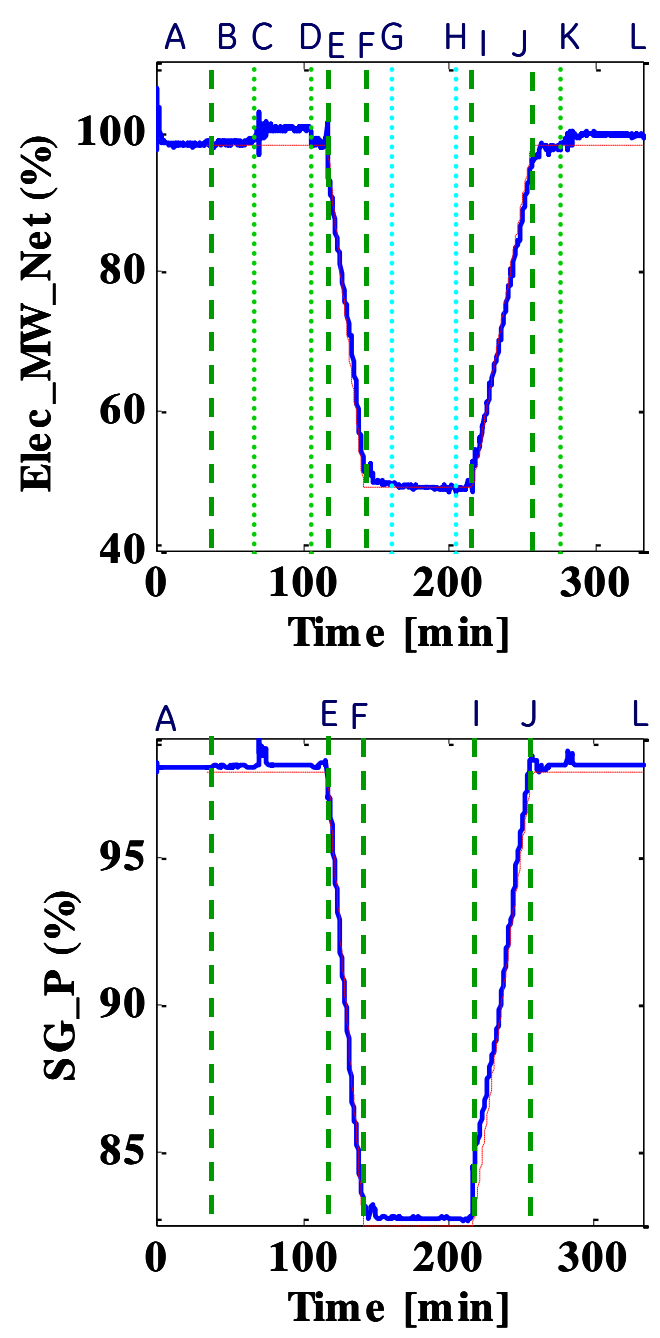

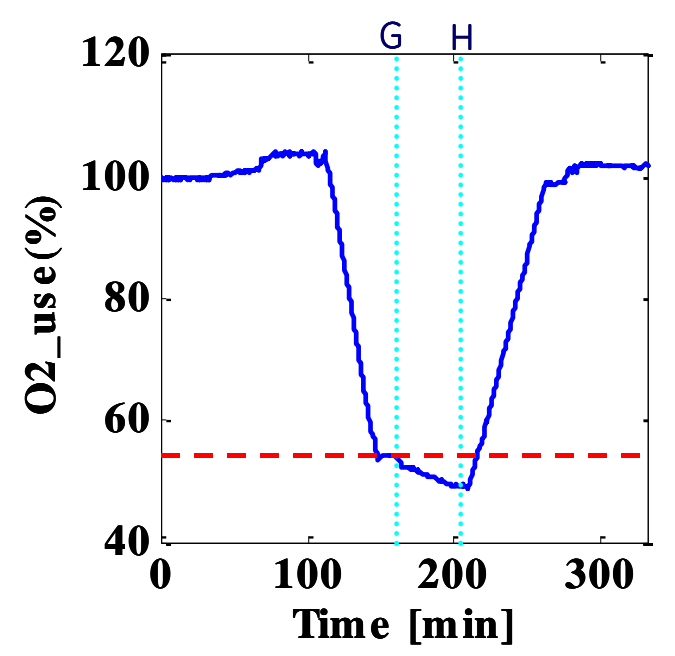

A : Simulation Starts with EKF engaged

$B$ : MPC engages

$B-C$ : Base load net MW tracking

C-D : Net MW maximization

A-E : Steady state Scrubber pressure set point tracking

E-F : 25\% faster ramp tracking (MW \& scrubber pressure)

$\mathrm{G}-\mathrm{H}$ : Oxygen use minimization mode engaged

I-J : 20\% faster ramp tracking(MW \& scrubber pressure)

$\mathrm{K}-\mathrm{L}$ : Net MW maximization

$\mathrm{J}-\mathrm{L}$ : Steady state Scrubber pressure set point tracking

Figure 67: Integrated sensing and control system closed loop response to load change from $100 \%-50 \%-100 \%$ with sensor and parameter errors with coal. The MW output and scrubber pressure (blue) track the reference profile (red) with $25 \%$ (ramp down) to $20 \%$ (ramp up) faster ramp rate compared to nominal.

Figure 68 shows the overall plant response with the integrated sensing and control system to change in load from base load to $50 \%$ part load and then back to base load in the manner as described earlier (Figure 67), in the presence of sensor (one random combination of sensor noise and bias) and modeling error (RSC fouling and Gasifier kinetics). For this simulation, the plant RSC is 30\% fouled and the gasifier kinetics is $70 \%$ of the nominal value. The EKF is initialized to nominal values of these parameters (RSC fouling $=0$ and gasifier kinetics scale factor $=1$ ). The simulation response shows that despite the presence of sensor noise and parameter error, the integrated sensing and control system has a good net electrical output set point tracking performance for both, at the steady state as well as the transient operation. This is similar to what was observed with state feedback MPC with ideal sensing. Further the integrated system is able to maximize the net electrical output for this combination of sensor noise and bias and model parameter error by more than $2 \%$ at the base load and cut down the oxygen consumption 
at partload by about $10 \%$. Figure 68 also shows that the EKF estimates of gasifier kinetics scale factor $\left(\mathrm{Eta}_{\mathrm{sf}}\right)$ and the RSC fouling factor $\left(\right.$ Fouling $\left._{\mathrm{sf}}\right)$ at base load condition are quite good. However, at part-load conditions due to limited "observability" of the parameters in the system, the parameter estimation is less accurate. More specifically, since EKF estimates all the states, parameters and biases such that combinations of all these result in minimum variance estimate to match the sensor measurements, the individual parameters are not guaranteed to track the actual parameters. However, the overall closed-loop response shows that even in the presence of sensor and modeling errors and despite the limited "observability" of the parameters in the system, the integrated sensing and control system is able to track the load and syngas pressure references well: tracking $20-25 \%$ faster turndown ramp rates. In the figure, the blue graph in Elec_MW_Net and SG_P represent the actual response whereas the setpoints are shown as red graph.
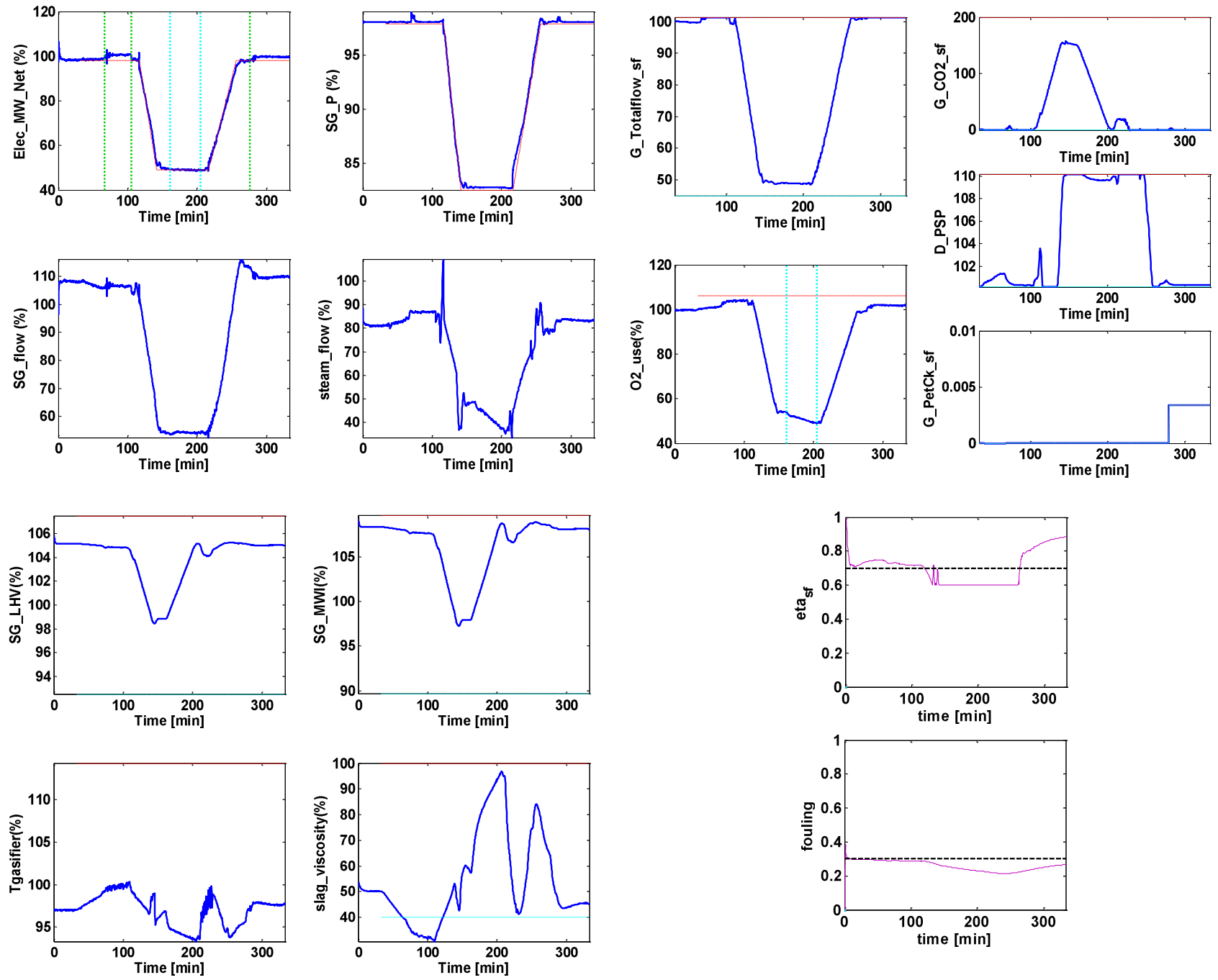

Figure 68: Integrated sensing and control system closed loop response to load change with coal from $100 \%-50 \%-100 \%$ with one combination of random sensor biases and parameter error.

As mentioned earlier, the designed integrated sensing and control closed-loop system was studied using Monte-Carlo simulations using random combinations of the sensor biases 
for each run. These results are summarized in Figure 69 and Figure 70. For all these simulations the plant RSC is $30 \%$ fouled and gasifier dynamics is $70 \%$ of the nominal value whereas, the estimator is initialized with nominal gasifier kinetics $\left(\mathrm{eta}_{\mathrm{sf}}=1\right)$ and no fouling in the RSC (fouling=0). Histograms of the maximum sustained net electrical power output gain at base load condition and maximum sustained saving in oxygen consumption at $50 \%$ part load observed in Monte-Carlo simulation are presented in Figure 69. The simulation shows that the gain in net electrical power output could be as much as $2-2.5 \%$, however, this gain is uneven and depends upon the sensor noise and bias combination. This is due to non-perfect observability in the system. Due to non-perfect observability some performance constraint parameters like carbon conversion, slag viscosity and Wobbe index cannot be estimated very accurately. This results in more conservative optimized performance due to constraints posed on these signals. The Monte Carlo simulation also shows that $7-10 \%$ sustained saving in oxygen consumption over the nominal consumption for $50 \%$ load can be achieved by judicious manipulation of control inputs. For consistency purpose, the saving in oxygen consumption is normalized with respect to the net electrical power output to account for any slight changes in electrical power output during the optimization of oxygen consumption phase. This gain is similar to what was observed with state feedback MPC with ideal sensors.

Finally, Figure 70 shows the time traces and histograms of the estimated values of gasifier kinetics parameter (eta $\left.{ }_{\mathrm{sf}}\right)$ and RSC fouling for the same Monte-Carlo simulations. As mentioned earlier, each simulation starts in open loop with model-based estimator (EKF) engaged for an hour before the controller (MPC) is activated. This is required for the estimates of "unknown" parameters in model (used both for EKF and MPC) and sensor biases to settle to its steady state values as seen in subplots in Figure 70 . Without this open loop operation, it was observed in multiple simulations that the interaction between the estimator and the controller might lead to closed loop system instability. The time traces of the parameters also show that these parameters are estimated more accurately at the base load steady state condition as compared with the part load steady state condition or during the transient operations, due to poor observability at part load conditions. Since the parameter observability is better at the base load condition, starting the simulation at these conditions also helps in more accurate estimation of these parameters. Starting at baseload condition, once the estimation has converged close to the "correct" value, it does not change significantly again on account of poor observability. The histograms at the bottom of Figure 70 correspond to the average value of corresponding parameters for each Monte-Carlo simulation. The figures show that the average parameter values over each simulation are close to actual values. 
Maximum Net MW Gain

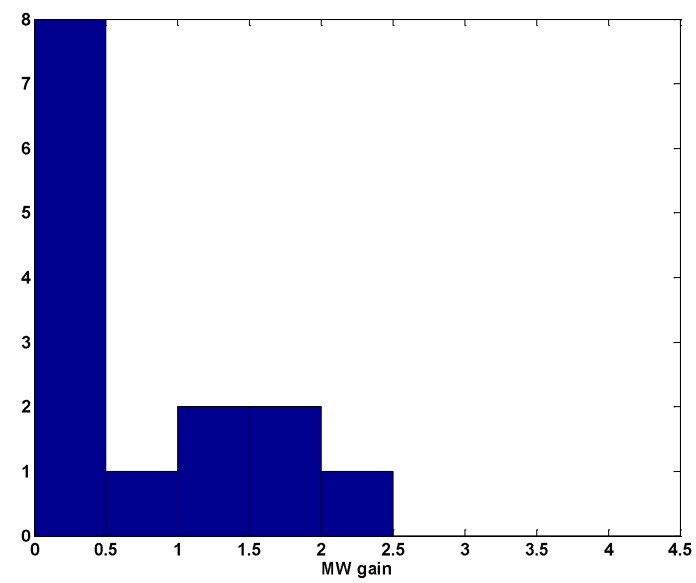

Maximum $\mathrm{O}_{2}$ saving

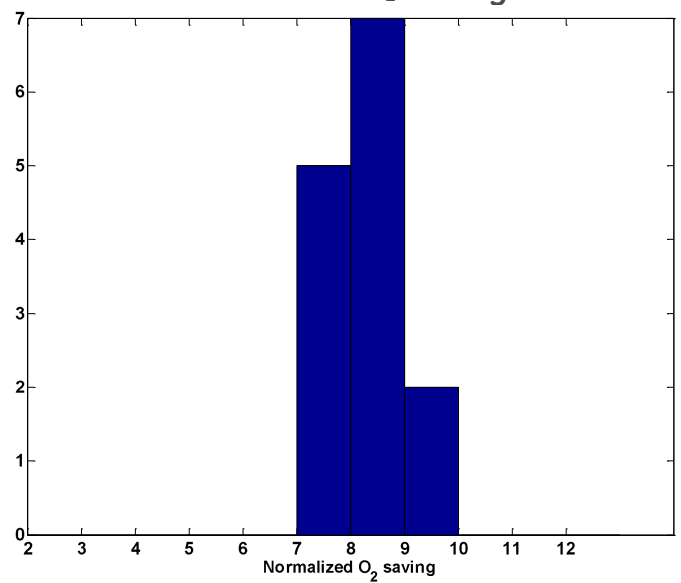

Figure 69: Monte Carlo simulation results for Net Electrical output gain at the base load condition and oxygen saving at $50 \%$ part load condition for various random combination of sensor error (noise and bias) and model parameter error (gasifier kinetics and RSC fouling) using the integrated sensing and control system for load changes from $100 \%-50 \%-100 \%$.
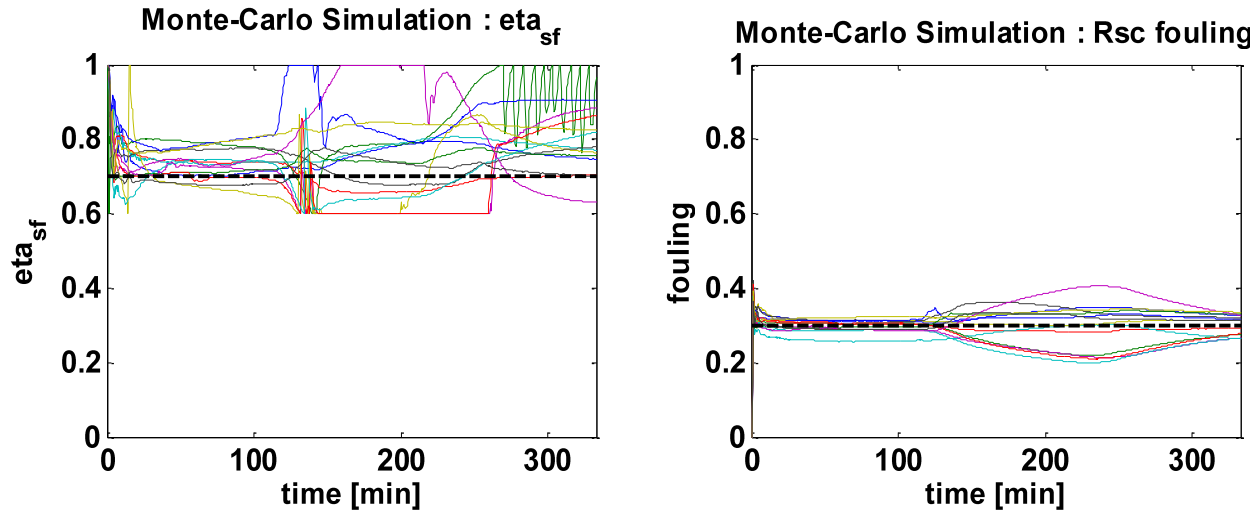

Histogram of average eta ${ }_{\text {sf }}$
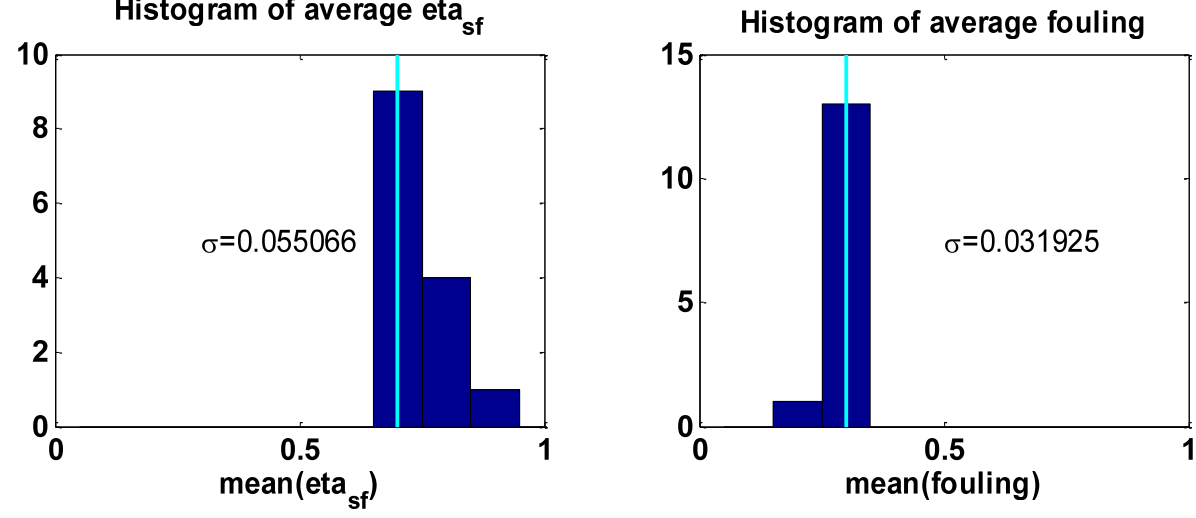

Figure 70: Monte Carlo simulation results for gasifier kinetics and RSC fouling for various random combination of sensor bias and model parameter errors for the integrated sensing and control system closed loop system to load change from $100 \%-50 \%-100 \%$. 


\subsubsection{Nominal Operation Optimization with Coal-Petcoke Blend}

Similar simulation studies were carried out for coal-petcoke fuel blend. These results are presented next. Figure 71 shows various phases of simulation with coal-petcoke fuel blend. The simulation is initiated (point ' $A$ ') at base load condition with EKF engaged. The simulation runs for almost 60 minutes without any external control input (open loop). After that the MPC is engaged at point ' $\mathrm{B}$ ', and it quickly tracks the net electrical output and scrubber syngas pressure to the respective reference set points. Operation phase ' $C$ ' to ' $D$ ' represents $25 \%$ faster ramp tracking in net MW as well as scrubber syngas pressure compared to the nominal operation to $50 \%$ part load. Again, since it is normally not desirable for the IGCC plant to turn down all the way to zero load due to startup complexities, the end of this turn down transient may represent the parking of the plant during the low demand period. During this period one objective would be to minimize the oxygen consumption while maintaining certain minimum load. The point 'E' represents starting of such a phase. At point ' $E$ ' the MPC is engaged in the minimization of the oxygen consumption mode while maintaining $50 \%$ load. At point ' $F$ ' the MPC disengages the oxygen minimization mode in preparation for transient operation. Phase ' $G$ '-' $\mathrm{H}$ ' of the simulation represents the ramp up transient tracking of net electrical output from $50 \%$ load to base load condition at $20 \%$ faster rate compared to the nominal rate. Once at base load, the MPC again engages tracking of net electrical output as well as scrubber syngas pressure mode and maintains the steady state operation. As mentioned, all through this simulation, the scrubber pressure is also tracked to a given reference trajectory as shown in the bottom graph of Figure 71. 

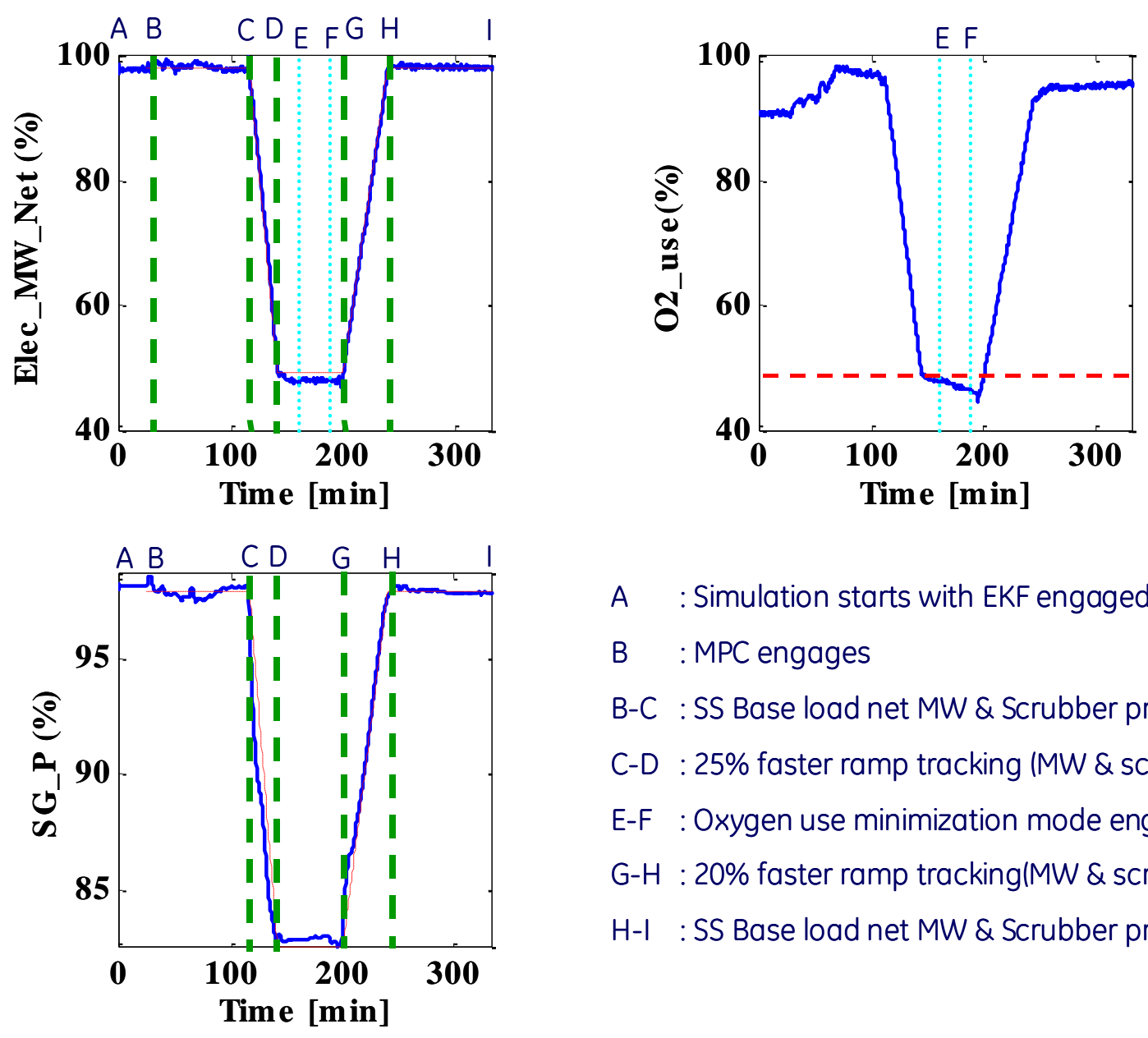

A : Simulation starts with EKF engaged

$B \quad$ : MPC engages

B-C : SS Base load net MW \& Scrubber pressure tracking

C-D : $25 \%$ faster ramp tracking (MW \& scrubber pressure)

E-F : Oxygen use minimization mode engaged

G-H : 20\% faster ramp tracking(MW \& scrubber pressure)

$\mathrm{H}-\mathrm{I}$ : SS Base load net MW \& Scrubber pressure tracking

Figure 71: Integrated sensing and control system closed loop response to load changes from $100 \%-50 \%-100 \%$ with sensor and parameter errors for coal-petcoke blend. The MW output (blue) track the MW reference (red) with 25\% (ramp down)-20\% (ramp up) faster ramp rate compared to nominal.

Figure 72 shows the overall plant response to the integrated sensing and control system to changes in load from base load to $50 \%$ part load and then back to base load in the manner as described earlier (Figure 71), in the presence of one random combination of sensor error (noise and bias) and modeling error (RSC fouling and gasifier kinetics). Similar to the coal case, for this study the plant RSC is 30\% fouled and the gasifier kinetics is $70 \%$ of the nominal value, while EKF is initialized to nominal values of these parameters (RSC fouling $=0$ and gasifier kinetics scale factor $=1$ ). The simulation response shows that despite the presence of sensor bias and parameter error the integrated sensing and control system has a good steady state as well as the transient operation tracking performance for both, the net electrical output as well as scrubber syngas pressure. This is similar to what was observed with state feedback MPC with ideal sensing. Further the integrated system is able to cut down the oxygen consumption by about $6 \%$ at partload. Figure 72 also shows the EKF estimates of gasifier kinetics scale factor $\left(\mathrm{Eta}_{\mathrm{sf}}\right)$ and the RSC fouling factor (Fouling $\mathrm{sf}_{\mathrm{sf}}$ ) over the duration of the simulation. The figure shows that plant parameters namely gasifier kinetics scale factor and RSC fouling factor estimates are slightly less accurate: at base load condition, the EKF estimates slightly faster gasifier kinetics whereas at the partload condition it estimates slightly slower 
kinetics. Further, at baseload condition the RSC fouling is accurately estimated close to the actual value of $30 \%$, while the estimation deteriorates at part load condition. This is due to limited "observability" of the parameters in the system, especially for small throughput at partload operation. Similar to coal case, since EKF estimates all the states, parameters and biases such that combinations of all these result in minimum variance estimate to match the sensor measurements, the individual parameters are not guaranteed to track the actual parameters. Nevertheless, the overall closed-loop response shows that even in the presence of sensor and modeling errors and despite the limited "observability" of the parameters in the system, the integrated sensing and control system is able to track the load and syngas pressure references well: tracking $20-25 \%$ faster turndown ramp rates. In the figure the blue graph in Elec_MW_Net and SG_P represent the actual response whereas the setpoints are shown as red graph.
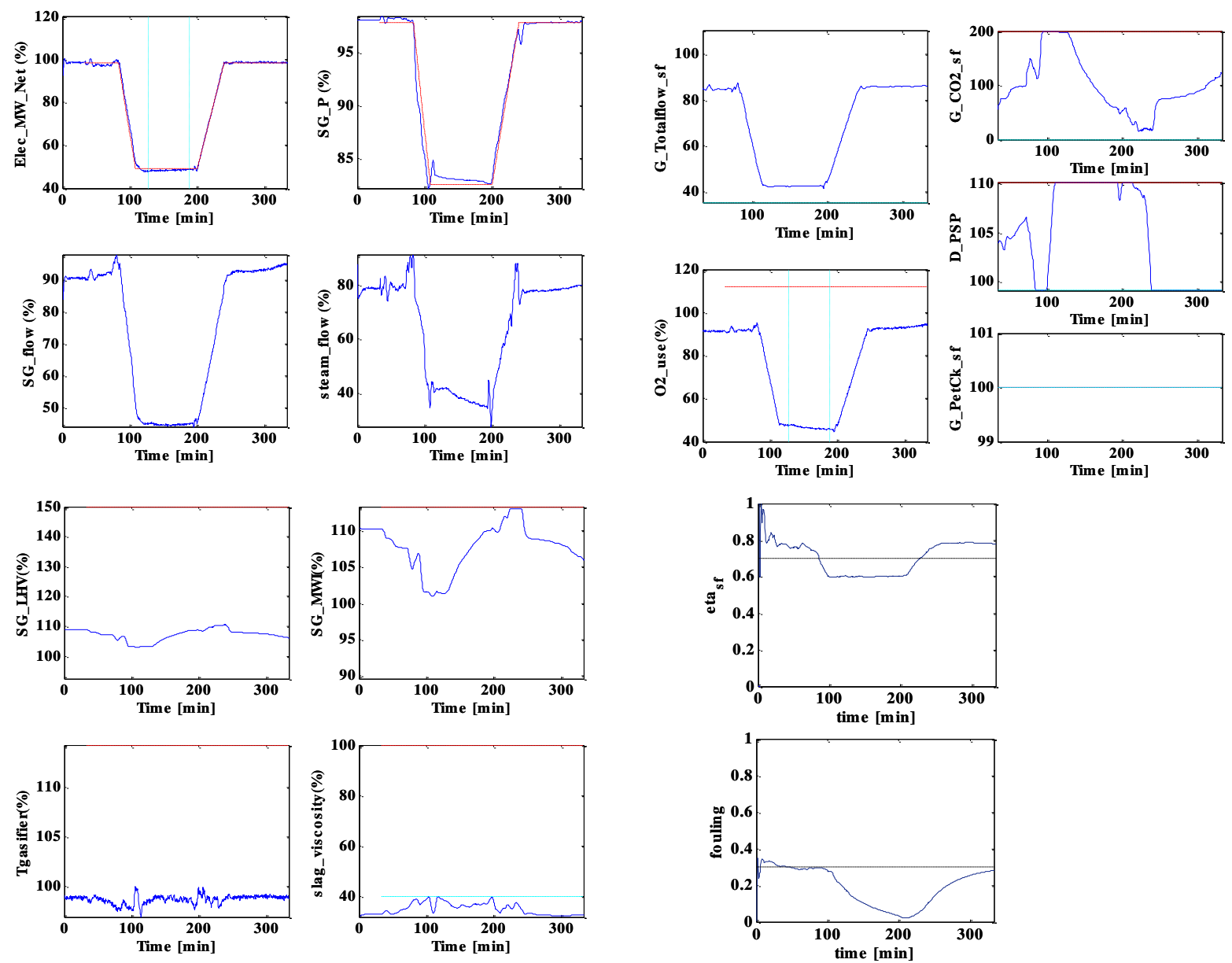

Figure 72: Integrated sensing and control system closed loop response to load changes from $100 \%-50 \%-100 \%$ for coal-petcoke blend with one combination of random sensor bias and parameter errors. 


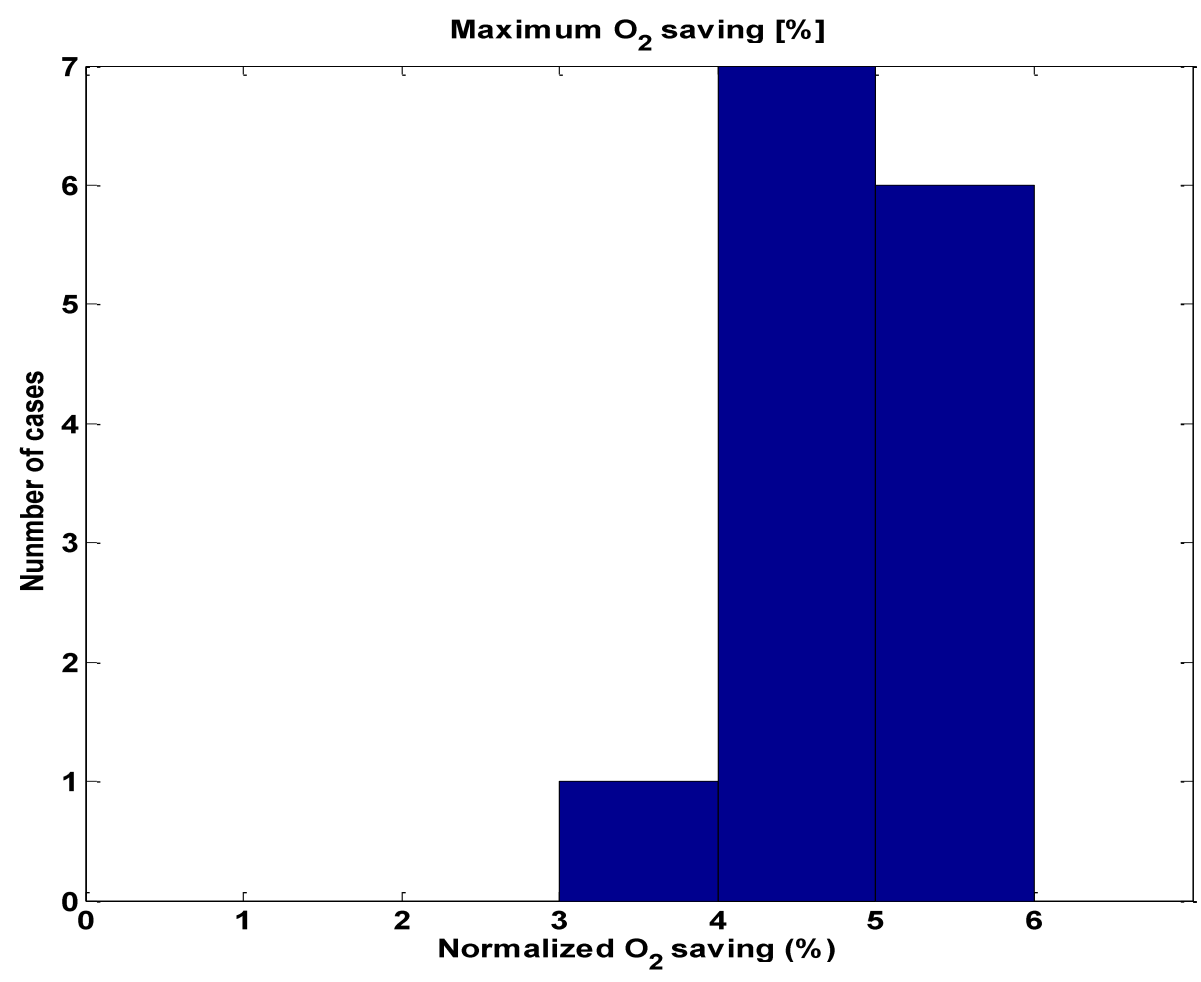

Figure 73: Monte Carlo simulation results for normalized oxygen saving at $50 \%$ part load condition with various random combination of sensors error (noise and bias) and model parameters error (gasifier kinetics and RSC fouling) for the integrated sensing and control system to load changes from $100 \%-50 \%-100 \%$.

As mentioned earlier, similar to coal fuel, the designed integrated sensing and control closed-loop system was studied using Monte-Carlo simulations using random combinations of the sensor biases for each run. These results are presented in Figure 73 and Figure 74. For all these simulations, the plant RSC is $30 \%$ fouled and gasifier kinetics is $70 \%$ of the nominal value whereas the estimator is initialized with nominal gasifier kinetics (eta sf $=1$ ) and no RSC fouling (fouling=0). Histogram of the maximum sustained saving in oxygen consumption at $50 \%$ part load observed in Monte-Carlo simulation is presented in Figure 73. The Monte Carlo simulation shows that 3-6\% sustained saving in oxygen consumption over the nominal consumption for $50 \%$ load can be achieved by judicious manipulation of control inputs for most cases of sensor bias combination. For some combinations of sensor bias, this gain may be quite small. This is due to non-perfect observability in the system. Due to non-perfect observability some performance constraint parameters like carbon conversion, slag viscosity and Wobbe index cannot be estimated very accurately. This results in more conservative optimized performance due to constraints posed on these signals. For consistency purpose, the saving in oxygen consumption is normalized with respect to the net electrical power output to account for any slight changes in electrical power output during the optimization of oxygen consumption phase. This gain is similar to what was observed with state feedback MPC with ideal sensors. 

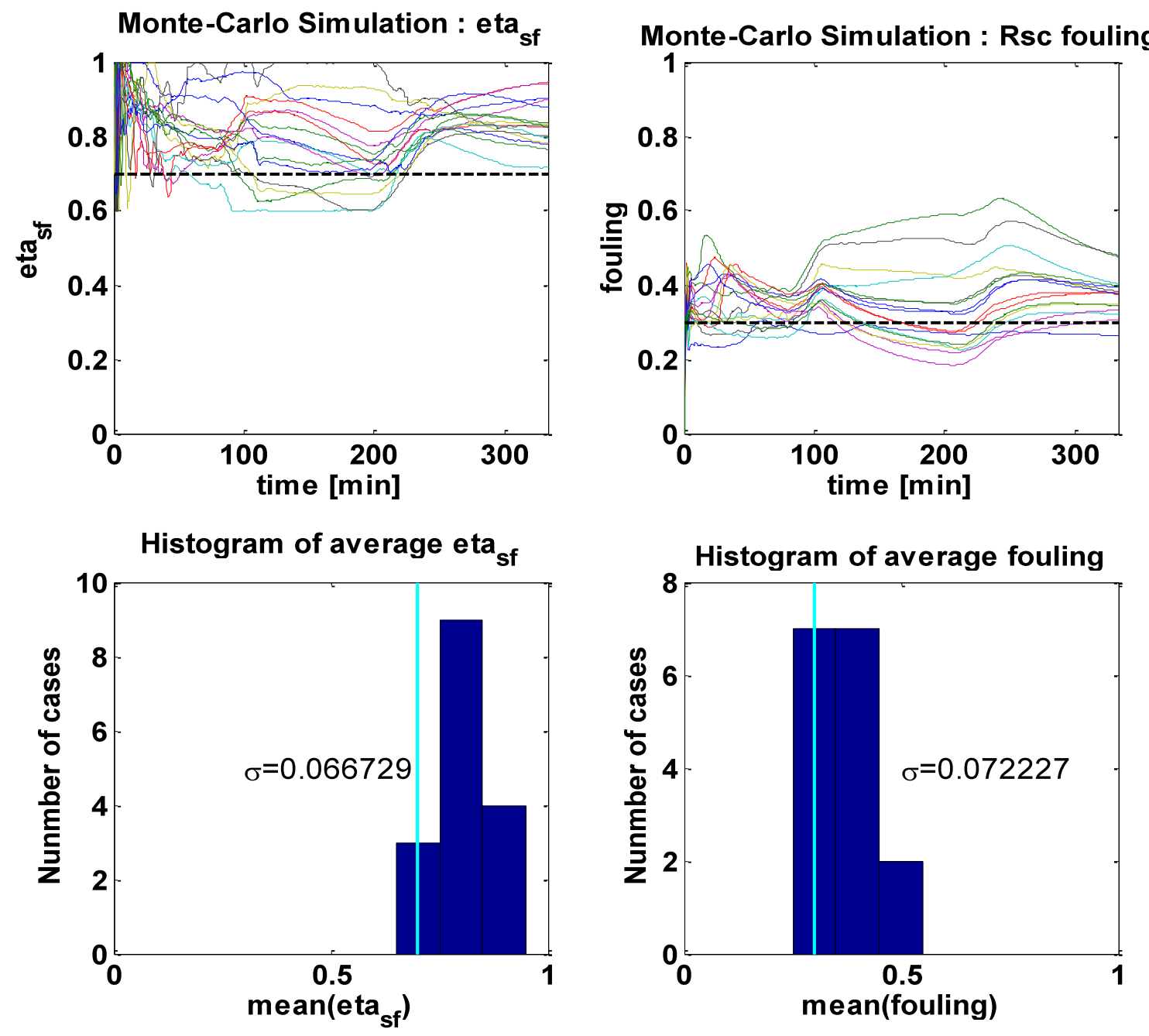

Figure 74: Monte Carlo simulation results for gasifier kinetics and RSC fouling for various random combination of sensor noise and model parameters error for the Integrated sensing and control system closed loop system to load changes from $100 \%-50 \%-100 \%$.

Finally, Figure 74 shows the time traces and histograms of the estimated values of gasifier kinetics parameter $\left(\mathrm{eta}_{\mathrm{sf}}\right)$ and RSC fouling for the same Monte-Carlo simulations. As mentioned earlier, each simulation starts in open loop with model-based estimator (EKF) engaged for an hour before the controller (MPC) is activated. This is required for the estimates of "unknown" parameters in model (used both for EKF and MPC) and sensor biases to settle to its steady state values as seen in subplots in Figure 74. Similar to coal fuel cases, without this open loop operation, it has been observed in multiple simulations that the interaction between the estimator and the controller may lead to closed loop system instability. The time traces of the parameters also show that these parameters are estimated more accurately at the base load steady state condition as compared with the part load steady state condition or during the transient operations, due to poor observability at part load conditions. Since the parameters observability is better at the base load condition, starting the simulation at these conditions also helps in more accurate estimation of these parameters. Starting at baseload condition, once the estimation has 
converged to the "correct" value, it does not change significantly again on account of poor observability. The histograms at the bottom of Figure 74 correspond to the average value of corresponding parameters for each Monte-Carlo simulation. The figures show that unlike the coal case, in coal-petcoke cases, the average parameter values over each simulation are less accurate. The performance of the EKF parameter estimation could possibly be improved by further retuning of the EKF. Despite this, as shown in Figure 72, the integrated sensing and control system shows good steady state as well as transient tracking performance and is able to optimize the operational cost within the operational constraints.

\section{Conclusions}

An IGCC plant is a large chemical plant, traditionally designed to operate mainly at steady state conditions, coupled to a power generation plant intended to operate in a robust, optimized and flexible manner to meet varying power generation demand. It is highly desired to achieve high degree of reliability and increasingly flexible operation in terms of turndown or load-following capability and fuel changes while achieving optimum overall plant efficiency. This in turn, implies a need for increasing automation for coordinated and optimized operation of the various sections of the plant to meet fluctuating power generation objectives. This program focused on developing advanced integrated sensing and control systems to achieve the objectives of higher reliability and flexible operation with optimized efficiency. In particular, this program focused on the gasification section, which is the core section of the plant, yet most limited in terms of automated operation, due in large part to a very harsh environment and corresponding limitations on online sensing. On the other hand, first-principles physics-based models have been developed for key process units, which are nominally used for offline analysis and design purposes. These physics-based models allow capturing the process operation knowledge in a rigorous manner and can be used online as the basis for design of an advanced sensing and control solution. Motivated by this, a systematic model-based approach for analysis and design of sensing and control systems has been developed in this program, and extensive simulation studies have been performed to identify the application and benefits of such an advanced sensing and control solution for online monitoring and control in an IGCC plant. The results and conclusions from each key element of the program are summarized in the next sections.

\subsection{Dynamic modeling for sensing and controls design}

It is clear that a good dynamic model based on first-principles is the foundation for design of advanced sensing and controls solution. However, the model has to be developed with the intended end-use for online sensing and control in mind. Important aspects for consideration include the level of details and time-scale of dynamics to be included, the computational efficiency and suitability for real-time simulation and estimation/controls, the flexibility and ability to adapt with adequate model parameters to address site-to-site design/operation variations as well as variations in a given plant over time. In particular, the model has to be adequately low-order to enable fast simulations as well as robust use in online sensing and controls. Similarly, instead of attempting to capture all minute details in the process and still have inaccuracies - there is always an approximation in any model

- it is often better to parameterize some aspects and allow room for online adaptation to 
changing process characteristics and keeping the updated model accurate. These aspects invariably lead to a trade-off in the model complexity and suitability for online monitoring and control.

With these considerations, in Phase I of the program, a unified dynamic model of the gasification section was implemented in Matlab/Simulink ${ }^{\circ}$ to be used for simulation studies as well as design of model-based sensing and control solutions. The models were implemented to describe both the nominal operation mode, as well as the phases of startup including pre-heating and post-ignition pressure ramp-up. In particular, a separate set of models was implemented for the gasifier refractory and RSC pre-heating, capturing the thermo-mechanical stress dynamics that limit the transient heating operation. The gasifier pre-heating model was implemented in Matlab/Simulink $\AA$ and ANSYS $\AA_{\text {. The }}$ available models for individual process units were leveraged to build the overall model. Where necessary, additional details were included, e.g. some models had only steadystate equations which were generalized to include dynamics, or in the case of the drum level model, additional details were included to better capture the "swell" effect during transients. On the other hand, the gasifier model was a high-order model that took up to twenty minutes or more to converge to a steady state - obviously not suitable for online sensing and control. This motivated the use of model-reduction to derive a low-order model of the gasifier that could be simulated within a fraction of a second while maintaining high accuracy with respect to the original high-order model over a wide operating envelope.

Often, once individual unit models are put together to describe the overall subsection or plant dynamics, invariably the overall model ends up being stiff, i.e. it has dynamics over a range of time-scales spanning fractions of seconds to minutes or hours. It is important only to keep the dynamics relevant for the characteristic time scale of the intended monitoring and control application. For instance, for the overall gasification section operation, which typically evolves overall several minutes, the gasifier dynamics occur over few seconds, which is too fast and can be ignored. Similarly, while in the full-order model used to simulate the "plant" detailed syngas flow and pressure dynamics were retained, these dynamics also occur over seconds and lead to stiffness. In order to enable fast simulation of the reduced-order model, especially for repeated future predictions for MPC, these pressure-flow dynamics were also approximated with quasi-steady-state approximations without sacrificing much accuracy.

Finally, in order to provide flexibility to the model and address uncertainties due to unknown (or un-modeled) aspects, parameters were defined in each process unit to allow for adapting the model and matching with real plant data. For instance, in the gasifier, the heterogeneous reactions are the rate-limiting reactions governing carbon conversion, and there is some uncertainty in the reaction kinetics - a kinetics scale factor was used to allow adjusting the kinetics in the presence of uncertainty. Similarly, in the RSC model the heat transfer varies with the degree of fouling which is introduced as a parameter to allow online adaptation based on available measurements. In the quench and the scrubber, a non-ideal VLE is used to capture the phase equilibrium between the syngas and the water holdup. To account for inaccuracies in the VLE relationship and potential limitations on gas-liquid equilibrium assumption, especially during transients, a temperature parameter was included to capture deviation from the modeled VLE relationship. These parameters 
are adapted online to match online sensor measurements, and keep the model matched to plant data.

In parallel to the model development, Phase I of the program also focused on implementation of sensors in the RSC in TECO Polk Power Station IGCC plant. Extensive lab tests were performed for fiber optic sensor design and packaging for temperature and strain sensing to meet the requirements for installation. In the second year of the program (Phase II), the sensor testing and packaging design and fabrication were completed. Finally, a set of radial temperature probes using Type B thermocouples and fiber optic FBG sensors, and fiber optic static strain sensors were installed in the RSC in TECO IGCC plant in 2009 Q1. The sensor packages worked very well, especially the temperature probes at level 10 in the RSC and the strain sensor in the RSC dome. The temperature probes at level 10 performed well, withstanding temperatures around $2000 \mathrm{~F}$, and multiple startup/shutdown transients. The sensors at level 7 also performed very well until the ceramic packaging broke abruptly on the fifth day of operation due to likely impingement of hot gas and/or slag. The strain sensor in the RSC probe worked very well, meeting all expectations and successfully measuring over approximately six weeks, a slowly increasing and small mechanical strain due to gradual fouling buildup, while withstanding very high thermal stresses $(\sim 5000-7000 \mu \varepsilon)$ due to high temperature conditions during startup. The FBG sensors performed very well for the intended short duration to collect data for model validation, despite the high level of challenges due to the extremely harsh operating conditions. Nevertheless, there is room for further improvement, especially in packaging design and installation, to enable robust sensors that can be used for long duration for monitoring and control.

\subsection{Advanced model-based sensing}

A key challenge in the monitoring and control of the core gasification section in an IGCC plant is the limited availability/survivability of sensors due to the extremely harsh environment. Owing to the limitations on available online sensors, a general model-based sensing approach wherein existing online sensors are coupled with online real-time modelbased estimation (or virtual sensing) provides an effective solution. Such an approach was developed in Phase II of the program under Task 2. The dynamic model for the gasification section was used in an EKF together with available online sensors, to estimate important unmeasured process variables important for monitoring and control, e.g. gasifier temperature, carbon conversion and slag viscosity.

A key concern in any sensing system is the accuracy of the sensor, whether it is real or virtual. In particular, the accuracy of the virtual sensor or model-based estimate depends on the accuracy of online (real) sensors and the model used for estimation. Initially, a linear model-based analysis was performed using Kalman filter design to identify key modeling errors (unknown/varying model parameters like gasifier kinetics, RSC fouling etc.) and sensor errors (unknown biases in sensors) that adversely impact the estimation accuracy. The ones with high sensitivity were included in the nonlinear model-based estimation using an EKF. A limitation of standard EKF is the inability to enforce inequality/bound constraints on the estimated "state" variables, e.g. mole fractions, fouling factor, which are expected to be in the range 0 to 1 . To address this, two different constraint handling techniques were implemented, one using post-update active constraint identification and correction, and another using preemptive correction for constraints 
before the measurement update. Through simulation studies, the latter approach was found to be more effective. However, there is room for improvement. In particular, the presence of constraints limits the effectiveness of underlying assumption of Gaussian variances assumed in an EKF, and techniques that relax this assumption would likely perform better. The performance of the constrained EKF was studied through extensive Monte Carlo simulations with random combinations of sensor biases, in the presence of modeling errors (errors in gasifier kinetics and RSC fouling) to show the estimation performance in the presence of these errors. Subsequently, in Phase III of the program this EKF was integrated with the MPC solution to obtain the overall integrated sensing and control solution. The simulation studies with the integrated sensing and control solution showed that in the presence of sensor errors, the unknown model parameters like gasifier kinetics and RSC fouling are more "observable", i.e. can be identified with higher accuracy, at baseload conditions as opposed to partload condition. This is consistent with the fact that at baseload conditions and corresponding high throughput, these parameters have higher sensitivity to the online sensors.

The ability to have a comprehensive online sensing/monitoring solution using a combination of limited online sensors complemented with model-based estimates is an important capability in itself. It allows real-time monitoring of process performance (e.g. gasifier temperature, carbon conversion), which can be used by plant operators for improved operation. When coupled with an advanced control solution, as done under Task 3 of the program, the integrated sensing and control solution achieves higher automation, robustness, flexibility and efficiency of plant operation.

\subsection{Advanced model-based control}

Finally, under Task 3, MPC controllers were designed for startup pre-heating of the gasifier refractory and RSC, as well as nominal operation with coal and coal-petcoke blends. MPC, through systematic online model-based future prediction and constrained optimization, allows achieving optimized plant operation while enforcing all safety, hardware and operability constraints. Also, MPC provides the ability to change the optimization objective (e.g. carbon conversion, oxygen use, efficiency, ramp rates) depending on the operation mode, thereby providing enhanced operation flexibility.

Initially, in Phase II, MPC was designed and tested through simulations for optimized startup pre-heating as well as nominal operation at steady state and through fuel and throughput/load transients. These MPC studies were performed assuming state feedback, or full knowledge of plant model states and outputs. During startup, gasifier and RSC preheating take significant time, consuming significant fuel as well as impacting plant availability. Faster preheating would benefit both. The MPC simulation studies with gasifier refractory preheating, subject to tensile and compressive thermal stress limits in the bricks, showed potential for $20 \%$ faster preheating compared to baseline open-loop schedule. Also, MPC studies were used to identify design tradeoff between desired faster preheating and reduction in thermal stresses. Similarly, MPC optimized operation for RSC pre-heating indicates a fast completion of pre-heating in less than ten hours, depending on the steam availability for pre-heating. MPC was used to perform extensive simulation studies for nominal operation at part-load and baseload conditions and for turndown between partload and baseload conditions. For coal-based operation, in one optimization mode at steady-state conditions, MPC yielded potential reduction of oxygen consumption by up to 
$8 \%$ at partload conditions. In another optimization mode for transient operation during turndown, MPC simulations yielded faster ramp-up and ramp-down capabilities by $20-25 \%$ faster than baseline capability due to the rate-limited ASU. Similarly, MPC simulations were used to study the transition from coal to coal-petcoke blends, and optimization for nominal operation at part-load and baseload conditions and turndown, yielding similar faster ramp rates during turndown and slightly reduced oxygen savings - the operation with coal-petcoke blend is more constrained compared to coal operation, partly due to the high carbon content in petcoke while still desiring a high carbon conversion. These improvements are achieved by MPC through coordinated use of multiple control inputs like slurry feed, oxygen feed, recycle $\mathrm{CO}_{2}$ feed and HP steam drum pressure, and controlled operation to constraint boundaries. These improvements in steady state and transient operation are significant.

In Phase III of the program, the EKF developed in Task 2 and MPC with state feedback from Task 3 were coupled to achieve the overall integrated sensing and control solution. In this effort, first the underlying physics-based models were updated to a common version, and then the EKF and MPC were coupled and re-tuned iteratively for good closed-loop performance of the integrated sensing and control solution. The physical model has many "unknown" parameters, e.g., gasifier kinetics, RSC fouling etc. The EKF provides estimates of these parameters, all model states as well as of outputs used by MPC and important for efficient and safe operation like the gasifier temperature, carbon conversion, slag viscosity etc. in the presence of random sensor noise and bias. This integrated solution was tested through Monte-Carlo simulations with random combinations of sensor biases in the presence of model parameter errors. The simulations were designed to test performance of the integrated sensing and control system for net electrical output and syngas pressure setpoint tracking at steady state and faster transients, as well as minimizing the oxygen use or maximizing electric power output at steady state, with coal as well as coal-petcoke fuel blend while respecting all the operating constraints.

The simulation studies showed that the steady state as well as the transient tracking performance of integrated system is good and comparable to the "ideal" MPC with state feedback in Phase II, despite the presence of sensor and modeling errors. Further, the integrated system provides the capabilities to the turn down $20 \%$ (ramp up) or $25 \%$ (ramp down) faster than the nominal operation while maintaining all the operational constraints. The integrated system also enables the optimization of various objective functions depending upon the current operation mode, thus providing operational flexibility. More specifically, for example, in the current setting, MPC was configured to maximize the net electric power output at base load condition, while minimizing the oxygen usage at the part load condition. The performance of the integrated sensing and control solution was studied through the Monte Carlo simulations. These simulations showed that the system net electrical output could be increased by up to $2-2.5 \%$ of the nominal value at the base load condition with coal slurry. Conversely, at partload operation, the oxygen consumption could be reduced by $7-10 \%$ for coal slurry. With coal-petcoke blend fuel, the oxygen saving at partload was a bit less at around 3-6\% - this is in part due to the more constrained operation with petcoke owing to the higher carbon content. The EKF also estimated the unknown model parameters like gasifier kinetics and RSC fouling. The estimates were good at baseload conditions, and deteriorated a bit during transients or at partload conditions. This is due to the fact that the parameters are more observable due to 
the higher sensitivity at baseload operation with higher throughput. The parameter observability is reduced at partload operation. Due to limited observability it is not always possible to estimate the unknown model parameters and compensate for sensor noise and bias accurately. Nevertheless, the integrated sensing and control system using EKF and MPC works very well for steady state and transient tracking as well as optimization.

The simulation studies have shown very promising results for the EKF and MPC implementations individually as well as for the final integrated EKF \& MPC solution. These results can be used as the basis for pursuing a future implementation of the developed sensing and controls solution in an IGCC plant, in a staged manner. One option is to first implement the EKF by itself using available online sensors in the gasification section and mature its performance in plant application - validating both the underlying model and the model-based estimation solution. As mentioned earlier, the EKF solution in itself is valuable for real-time online monitoring of the plant performance that can be used by plant operators for improved operation. Once the performance of the model and the EKF-based sensing solution is established, MPC can be implemented and integrated with EKF to achieve the advanced sensing and control solution. Again, MPC could initially be used in an "advisory" mode to aid operators improve the plant operation. Subsequently, as its performance is established, it could be implemented in a closed-loop mode to enable automated operation and achieve the performance improvement entitlement. In a separate direction, the developed advanced sensing and control solution for the gasification section could be expanded or integrated with sensing and controls for the other sections, including the ASU, syngas cleanup and power generation towards an overall plant-level control system for optimizing the overall IGCC plant operation.

\section{Abbreviation}

This section lists the abbreviations used in this report.

$\begin{array}{ll}\text { ASU } & \text { Air Separation Unit } \\ \text { CSC } & \text { Convective Syngas Cooler } \\ \text { EKF } & \text { Extended Kalman Filter } \\ \text { FBG } & \text { Fiber Bragg Grating } \\ \text { IGCC } & \text { Integrated Gasification Combined Cycle } \\ \text { KF } & \text { Kalman Filter } \\ \text { LHV } & \text { Lower Heating Value } \\ \text { MPC } & \text { Model Predictive Control } \\ \text { MWI } & \text { Modified Wobbe Index } \\ \text { POD } & \text { Proper Orthogonal Decomposition } \\ \text { RSC } & \text { Radiant Syngas Cooler } \\ \text { SBL } & \text { Soot Blower Lance } \\ \text { UKF } & \text { Unscented Kalman Filter }\end{array}$

